\title{
Surface-brightness fluctuations in stellar populations ${ }^{\star}$
}

\section{IAC-star models for the optical and near-IR wavelengths}

\author{
A. Marín-Franch ${ }^{1}$ and A. Aparicio ${ }^{2}$ \\ 1 Department of Astronomy, University of Florida, 211 Bryant Space Science Center, Gainesville, FL 32611, USA \\ e-mail: amarin@astro.ufl.edu \\ 2 Departamento de Astrofísica, Universidad de La Laguna; and Instituto de Astrofísica de Canarias, Calle vía Láctea s/n, 38200 La Laguna, \\ Tenerife, Spain \\ e-mail: antapaj@iac.es
}

Received 20 July 2005 / Accepted 14 December 2005

\section{ABSTRACT}

Aims. A new theoretical calibration of surface-brightness fluctuations (SBF) for single age, single metallicity stellar populations is presented for the optical and near-IR broad-band filters, as well as for the HST WFPC2 and ACS filters.

Methods. The IAC-star code is used. Two Padua and the Teramo stellar evolution libraries have been considered. A set of single-burst stellar populations (SSP) with a wide range of ages $(3 \mathrm{~Gy}-15 \mathrm{~Gy})$ and metallicities $(Z=0.0001-0.03)$ have been computed using each one of the three considered stellar evolution libraries. For each SSP, color indexes and SBF magnitudes are given for the filters $U, B, V, R, I, J, H, K, F 218 W$, $F 336 \mathrm{~W}, F 439 \mathrm{~W}, F 450 \mathrm{~W}, \mathrm{~F} 555 \mathrm{~W}$ and $F 814 \mathrm{~W}$, and for the first time, an uncertainty has been estimated for the SBF theoretical calibration.

Results. Although some differences might be addressed, the Padua and Teramo stellar evolution libraries provide comparable SBF results. A detailed comparison of the present SBF calibrations with both previous calibrations and observational data is also presented.

Comparing the different models with observational data, Padua based models reproduce fairly well the optical data for globular clusters, while Teramo based models fits both optical galaxies and globular clusters data, as well. In the near-IR wavelengths, the Teramo based models provide the only SBF theoretical calibration to date able to properly reproduce the observational data for superclusters, with intermediate-to-low metallicity.

As a conclusion, Teramo based models work better than any other calibration reproducing observational data for the near-IR wavelengths. Furthermore, the age-metallicity degeneracy is broken for low metallicity $(Z \leq 0.0037)$ stellar populations. Finally, a clear relation between the $B \mathrm{SBF}$ absolute magnitude of a stellar population and its metallicity is found for intermediate to old populations, so the $B$-band fluctuation magnitude is proposed as a metallicity tracer. The present theoretical calibration shows that the analysis of SBF provides a very powerful tool in the study and characterization of unresolved stellar populations.

Key words. galaxies: distances and redshifts - galaxies: elliptical and lenticular, cD - galaxies: fundamental parameters - galaxies: star clusters - galaxies: stellar content

\section{Introduction}

The concept of surface-brightness fluctuations (SBF) was introduced by Tonry \& Schneider (1988), who noted that in the surface photometry of a galaxy far enough to remain unresolved, a pixel-to-pixel fluctuation is observed due to the Poisson statistics of the spatial distribution of stars. They developed SBF for its use as an extragalactic distance indicator for early-type galaxies. The ratio of the second moment to the first moment of

* All Tables and Figs. 5-9 , 11, 13 and 19 are only available in electronic form at http://www. edpsciences.org the stellar luminosity function of the galaxy is used as a standard candle:

$\bar{L} \equiv \frac{\sum n_{i} L_{i}^{2}}{\sum n_{i} L_{i}}$

where $n_{i}$ is the number of stars of type $i$ and luminosity $L_{i}$. Since SBF depend on the second moment of the stellar luminosity function, they are especially sensitive to the most luminous stars which, in an intermediate to old stellar population, are the red giants. For the same reason, SBF are relatively insensitive to differences in the initial mass function (IMF) for intermediate to old stellar populations (Worthey 1993; 
Liu et al. 2000; Blakeslee et al. 2001; Cantiello et al. 2003). The quantity $\bar{L}$ has units of luminosity. The absolute and apparent magnitudes of $\bar{L}$ are $\bar{M}$ and $\bar{m}$, respectively. The apparent fluctuation magnitude $\bar{m}$ can be observationally measured, and if the distance to the target galaxy is known, $\bar{M}$ can be obtained. As SBF are an intrinsic property of a stellar population, they can be used to study some characteristics of the stellar populations of a galaxy, such as age and metallicity as well, and not only for distance measurements.

Several attempts have been done to compute SBF magnitudes using population synthesis. First studies appeared in the early 90's with works by Tonry et al. (1990), Worthey (1993) and Buzzoni (1993). More recently, the use of SBF in stellar population synthesis has received renewed interest with the works of Liu et al. (2000, hereafter LCG00), Blakeslee et al. (2001, hereafter BVA01) and Cantiello et al. (2003, hereafter C03). They presented SBF theoretical calibrations as a means for probing stellar populations and as an independent check of the Cepheid distance scale, and for age-metallicity studies of unresolved stellar populations. LCG00 presented SBF model predictions using the latest version of the Bruzual-Charlot population synthesis models (Bruzual \& Charlot 2000), a completely updated version of the Bruzual \& Charlot (1993) models. For metallicities ranking from $1 / 200$ to 2.5 times solar, the stellar evolution is computed following the prescription of the Padova school (Bressan et al. 1993). BVA01 used an updated version of the Vazdekis et al. (1996) code, making use of the homogeneous sets of stellar evolution models by Bertelli et al. (1994) and Girardi et al. (2000). C03 used the stellar evolutionary tracks from the Teramo-Pise-Rome group, based on the evolutionary stellar code FRANEC (Cassisi et al. 1998).

On the other hand, the major observational effort on SBF has been focused on SBF measurements for distance determinations. Tonry et al. (2000, 2001), based on observations of 300 early-type galaxies, provided the best $I$-band SBF empirical calibration to measure accurate distances to nearby galaxies. In the near-IR, Liu et al. (2002) presented a $K$-band SBF empirical calibration for distance measurements based on observations of 19 early-type galaxies in the Fornax cluster. More recently González et al. (2004) and González-Lópezlira et al. (2005) presented the first attempt to empirically calibrate the near-IR SBF method. They used the co-adding of globular clusters with similar age and metallicity, called "Superclusters", to compare empirical SBF with models predictions.

These efforts underscore the importance of a solid theoretical calibration of the SBF method. A comparison of the latest theoretical SBF calibrations reveals that LCG00, BVA01 and $\mathrm{C} 03$ results are not compatible. This highlights the need for a fully consistent SBF theoretical calibration.

The new IAC-star code (Aparicio \& Gallart 2004) for synthetic color-magnitude diagram (CMD) computation offers significant improvement in synthetic stellar population computations, and therefore allows to perform a new SBF theoretical calibration. In this paper, a new theoretical calibration of stellar population SBF in the optical and near-IR wide band filters is presented, as well as for the HST WFPC2 and ACS filters. The theoretical calibration has been performed for both the Padua (Bertelli et al. 1994; Girardi et al. 2000) and Teramo
(Pietrinferni et al. 2004) stellar evolution libraries. This offers the opportunity to compare up to three different synthetic stellar population models with the previous works, as well as with the observational data, bringing us nearer to a fully consistent theoretical calibration of SBF in stellar populations.

\section{Stellar population synthesis}

\subsection{IAC-star: the code}

Stellar population synthesis research requires a few basic ingredients (see Gallart et al. 2005, for a review). If a synthetic stellar population is to be computed, the Star Formation History $(\mathrm{SFH})$, describing the characteristics of the stellar system and their evolution with time, must be defined. This is determined by the Initial mass function (IMF), the Star formation rate [SFR $(t)]$, the Chemical enrichment law $[Z(t)]$ and Frequency and distribution of binary stars. Once the SFH is defined for the synthetic stellar population, a stellar evolution library must be used to evolve the stellar population and obtain its present time physical parameters (effective temperature of every remaining star, $T_{\text {eff }}$, its luminosity, $L$, and its gravity, $g$ ). Different stellar evolution libraries use different input parameters and physics. Main characteristics of a stellar evolution library are the mass and metallicity range of stars that can be considered, the evolutionary phases computed, and the assumed model physics (equation of state, nuclear reactions rates, opacity, neutrino energy losses, model atmosphere). Other parameters, like mixing length (efficiency of convection) and mass loss are not well known, so they must be fine-tuned comparing model predictions with observational data. Once the physical parameters of every single star in the considered synthetic stellar population are obtained, their magnitudes and colors must be computed. A bolometric correction library must be used to translate $T_{\text {eff }}$, $L$ and $g$ into colors and magnitudes. A bolometric correction library uses model atmospheres that provide synthetic spectra for a grid of $T_{\text {eff }}, g$ and $Z$. Synthetic spectra is then convolved with a filter response curve, providing colors and magnitudes for all stars in the stellar population. Different stellar population synthesis models use various combinations of the previous main ingredients, together with different computation tools and approaches.

The IAC-Star code presents a new algorithm for the computation of synthetic CMD. It is mainly aimed at star formation history studies in nearby galaxies. Although it is designed to generate synthetic CMDs, IAC-Star also provides integrated and SBF magnitudes and colors for the total synthetic stellar population, so can be used for populations synthesis research.

Stellar populations are calculated on a star-by-star basis. $L$, $T_{\text {eff }}$, and $g$ of each star are computed by direct bilogarithmic interpolation in both the metallicity and age grids of a library of stellar evolution tracks, metallicity and age following continuous distributions. All the stellar evolution phases are covered accurately, and mass loss is computed during the red giant branch (RGB) and the asymptotic giant branch (AGB) phases (see Aparicio \& Gallart 2004, for details). A set of different SFR, IMF and $Z(t)$ are allowed, and binary star content can be computed. The result is a synthetic CMD with smooth, realistic 
stellar distribution. This significantly improves the accuracy of the results as compared with methods without such interpolations.

IAC-Star allows the user to choose between three different stellar evolution libraries: the two Padova (Bertelli et al. 1994; Girardi et al. 2000) and the Teramo (Pietrinferni et al. 2004) stellar evolution libraries. Besides the physical parameters, photometric data is also provided for each star by means of a bolometric corrections library. The user can choose between four different bolometric correction libraries. In this work, the one from Castelli \& Kurucz (2003) has been used for the integrated broadband colors and magnitudes in the standard UBVRIJHK filters, and Origlia \& Leitherer (2000) for the HST WFPC2 and ACS filters. The IAC-Star code also provides integrated visual (broadband and Hubble Space Telescope) and infrared magnitudes and SBF magnitudes for the total synthetic stellar population.

In this paper, we focus on the SBF predictions of the stellar population synthesis code for different stellar evolution and bolometric correction libraries.

\subsection{Single burst stellar population synthesis}

The idea behind this calibration is to develop a tool for unresolved stellar population research. This tool is being implemented through a number of calibration grids, or "Fluctuation Color-Magnitude Diagrams". These are diagrams of integrated colors and SBF magnitudes for a set of theoretical single-burst stellar populations (SSP). Each SSP is represented by a point in the diagram and the plot is completed by a grid of lines connecting same age and same metallicity SSP. Plotting real SBF results from observations onto them will provide estimations of age and metallicity for the observed stellar population.

In order to build the calibration grids, two Padua stellar evolution libraries, Bertelli et al. (1994, hereafter B94) and Girardi et al. (2000, hereafter G00), and the Teramo stellar evolution library by Pietrinferni et al. (2004, hereafter P04) are considered, together with the two bolometric correction libraries by Castelli \& Kurucz (2003, for the optical and nearIR wavelengths) and Origlia \& Leitherer (2000, for the HST WFPC2 and ACS filters). It is worth noting that B94 stellar evolution library has been completed with Girardi et al. (1996) for $Z=0.0001$, and that the same bolometric corrections are used for the three considered stellar evolution libraries, so possible differences between the three model predictions should be attributed to differences in the stellar evolution libraries, rather than in their transformations to observational quantities. The Kroupa IMF (Kroupa et al. 1993) has been used. Changes in the IMF would have a negligible effect in the SBF results for old ( $\geq 5$ Gyr) stellar populations (Worthey 1993; Liu et al. 2000; Blakeslee et al. 2001; Cantiello et al. 2003).

For B94 and G00 stellar evolution libraries, a set of SSP with ages in the interval from 3 Gy to 15 Gy have been computed. On the other hand, P04 models for low mass stars (below 0.8 Solar masses) are followed only until 16 or 17 Gyr. Since IAC-star does not extrapolate, for some very old, very low mass stars the older data lack for the interpolation and so

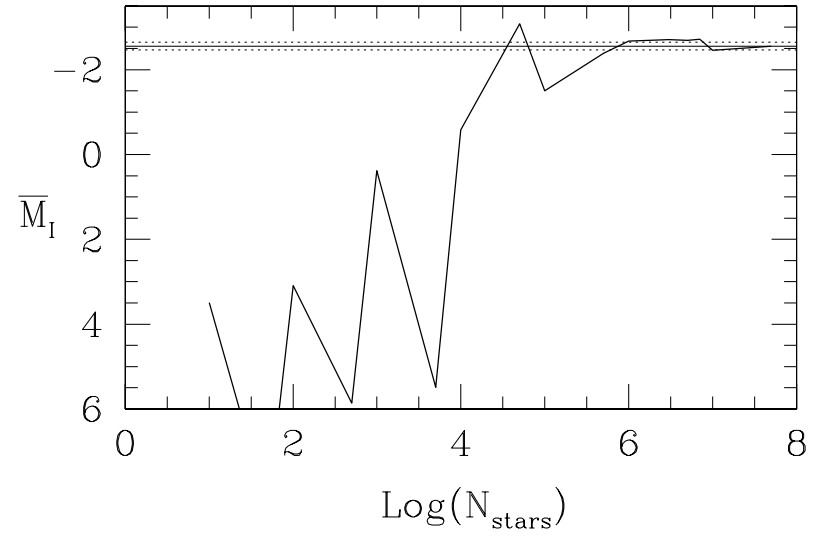

Fig. 1. SBF result for the $I$ filter as a function on the number of computed stars $\left(\log \left(N_{\text {stars }}\right)\right)$. B94 based models with $Z=0.0018$ and $7 \mathrm{Gyr}$ have been considered for this plot. The final $\bar{M}_{I}$ value has been plotted as well (solid line), together with its computed uncertainty (dotted lines). It can be seen that for simulations with $N_{\text {stars }}$ bigger than $10^{6}$, the SBF result is stable and similar to the final $\bar{M}_{I}$ value, within the error bar.

the synthetic star is not computed. To avoid biases and incompleteness, stellar population models including stars older than 14 Gyr are not allowed by the code when the possibility exists of some stars being computed and some of them not. For this reason, a set of SSP with ages in the interval from 3 Gy to 13 Gy have been considered for this stellar evolution library. For all considered ages, metallicities from $Z=0.0001$ to $Z=0.030$ have been computed using B94 and P04 models, and from $Z=0.0004$ to $Z=0.030$ using G00.

As the SBF signal is dominated by the brightest stars of the stellar population, if the number of computed stars per SSP is low then stochastic effects from those brightest stars become important, being the computed SBF magnitudes completely dominated by those stochastic effects. For this reason, a large number of stars is necessary to accurately compute SBF magnitudes. The effect of the number of stars on SBF magnitudes can be clearly seen in Fig. 1 . In this figure, the $I$-band SBF magnitude is plotted as a function of the number of stars used in the synthetic stellar population $\left(N_{\text {stars }}\right)$. A SSP with $Z=0.0018$ and an age of $7 \mathrm{Gyr}$ has been considered for this plot. It can be seen that the SBF signal is completely random for $N_{\text {stars }}$ lower than $10^{5}$. The SBF magnitude becomes stable for $N_{\text {stars }}$ bigger than $10^{6}$. For higher number of stars, the SBF I magnitude oscillates around the final computed value, between its error bars.

In this work, each SSP is composed by $5 \times 10^{7}$ stars, but instead of computing every SSP in a single simulation, five simulations with $10^{7}$ stars have been performed for every age and $Z$ pair. This way, the final SBF magnitudes and their uncertainties of an SSP can be obtained as the mean \pm standard deviation of its five corresponding simulations. In Fig. 1, it can be seen that the obtained I-band SBF magnitude and its uncertainty clearly reproduces the SBF results for SSP with $N_{\text {stars }}$ bigger than $10^{6}$.

The total number of computed simulations has been 1500 , each one containing $10^{7}$ stars. Such a high number of simulations has given the opportunity not only to compute stable SBF magnitudes for the 300 considered SSPs, but to present for the 


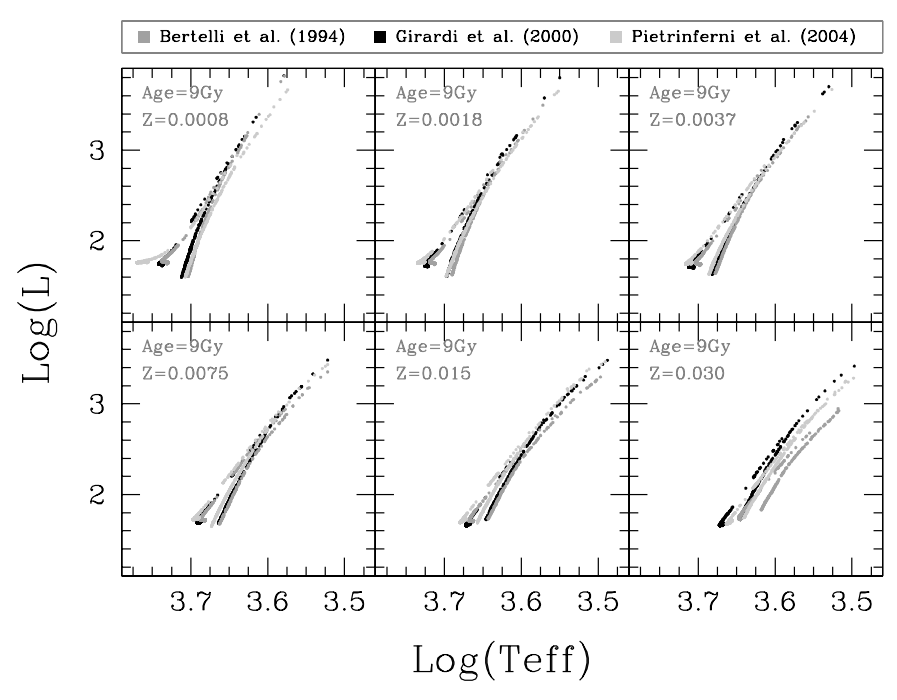

Fig. 2. 9 Gyr theoretical isochrones obtained using the stellar evolution libraries by B94, G00 and P04, respectively. Only stars brighter than $M_{V}=1$ are plotted. It can be seen that the three stellar evolution libraries show similar isochrones in all the metallicity range, except for the high metallicity extreme.

first time an estimation of the SBF theoretical calibration uncertainties as well.

\section{Results}

SSP integrated colors and SBF magnitudes, as well as the error estimates for the three libraries are shown in Tables 1 to 6 , for the optical and near-IR standard filters. Tables 1 and 2, show results obtained with the stellar evolution library of B94, Tables 3 and 4, with the G00 stellar evolution library, and Tables 5 and 6 , with the stellar evolution library by P04. On the other hand, HST WFPC2 and ACS model predictions are listed in Tables 7 to 12 . Tables 7 and 8 show results obtained with the stellar evolution library of B94, Tables 9 and 10 with the G00 stellar evolution library, and Tables 11 and 12, with the stellar evolution library by P04.

Before studying in detail the SBF results, an example of theoretical isochrones obtained using the three different stellar evolution libraries have been analyzed. Figure 2 shows 9 Gyr isochrones obtained for different metallicity values and using the stellar evolution libraries by B94, G00 and P04, respectively. As SBF are mainly produced by the most luminous stars of the stellar population, only stars brighter than $M_{V}=1$ are plotted. It can be seen that the three stellar evolution libraries show similar isochrones in all the metallicity range, except for the high metallicity extreme. For $Z=0.030$ for example, the RGB of B94 is significantly fainter than the RGB of the other sets of stellar evolution models, but the color is basically the same. If only the brightest stars are taken into account, the brightest stars of the B94 stellar evolution library are fainter and slightly bluer. As the applied transformations to the observational plane are the same, one should expect similar integrated colors for the three stellar evolution libraries but fainter and bluer SBF magnitudes for the B94 based models, as we will show later.
In order to compare the three sets of models in all the considered age and metallicity ranges, examples of SBF model grids are plotted in Figs. 3 to 5. These three figures show different examples of "fluctuation color-magnitude diagrams" (FCMD) in the optical, the near-IR and for the HST WFPC2 and $A C S$ filters.

Figure 3 shows the FCMD obtained using respectively the stellar evolution libraries by B94, G00 and P04. $\bar{M}_{B}$ and $\bar{M}_{I}$ SBF magnitudes have been chosen for this plot, as an example. Lines represent SSP with same age or metallicity. Each SSP is the result of averaging five models with $10^{7}$ stars each. It can be seen that the three stellar evolution libraries provide comparable results, although the G00 and P04 based model results are slightly redder than those based on B94 for SSP younger than $\sim 5-7$ Gy and for SSP in the high metallicity limit, as expected after analyzing the model isochrones in Fig. 2. The agemetallicity degeneracy is broken in all cases for low metallicity $(Z \leq 0.0037)$ stellar populations.

Figure 4 shows the same as Fig. 3 , but for $\bar{M}_{J}$ and $\bar{M}_{K}$ SBF magnitudes, as an example of near-IR wavelengths. Again, the three stellar evolution libraries provide comparable results, although the G00 and P04 based model results are slightly redder than those based on B94. It can be seen that the nearIR provides better age-metallicity resolution than the optical wavelengths, and the age-metallicity degeneracy is broken in all cases for low metallicity $(Z \leq 0.0018)$ stellar populations. Figure 5 shows the same as Fig. 3 , but in this case $\bar{M}_{F 450 W}$ and $\bar{M}_{F 814 W}$ SBF magnitudes have been chosen as an example of HST WFPC2 and ACS filters. As in Figs. 3 and 4, the three stellar evolution libraries provide comparable results, being the G00 and P04 based model results are slightly redder than those based on B94. Again, the age-metallicity degeneracy is broken in all cases for low metallicity $(Z \leq 0.0037)$ stellar populations. This proves SBF analysis to be a powerful tool to study low metallicity stellar populations.

It is worth noting that the computed SBF model prediction's uncertainties are negligible to those from varying stellar evolution models. In other words, the differences in the SBF model predictions obtained using B94, G00 and T04 are bigger than the computed SBF uncertainties for each one of the three theoretical calibrations. So the differences in the SBF predictions using different stellar population libraries, although small, are real.

A different kind of useful figure that can be plotted is integrated color versus fluctuation color. In Fig. 6, $(B-I)$ is plotted as a function of $\left(\bar{M}_{B}-\bar{M}_{I}\right)$ using respectively the stellar evolution libraries by B94, G00 and P04. Lines represent SSP with same age or metallicity. This plot provides less resolution that the one shown in Figs. 3 to 5, but having the advantage of being distance independent. So it can be very useful in those cases where distance is unknown or not well determined.

Summarizing, the three different stellar evolution libraries provide comparable FCMDs, making the present theoretical SBF calibration fully consistent. Besides, the age-metallicity degeneracy is broken for low metallicity $(Z \leq 0.0037)$ stellar populations, proving SBF analysis to be a powerful tool to study low metallicity stellar populations. 

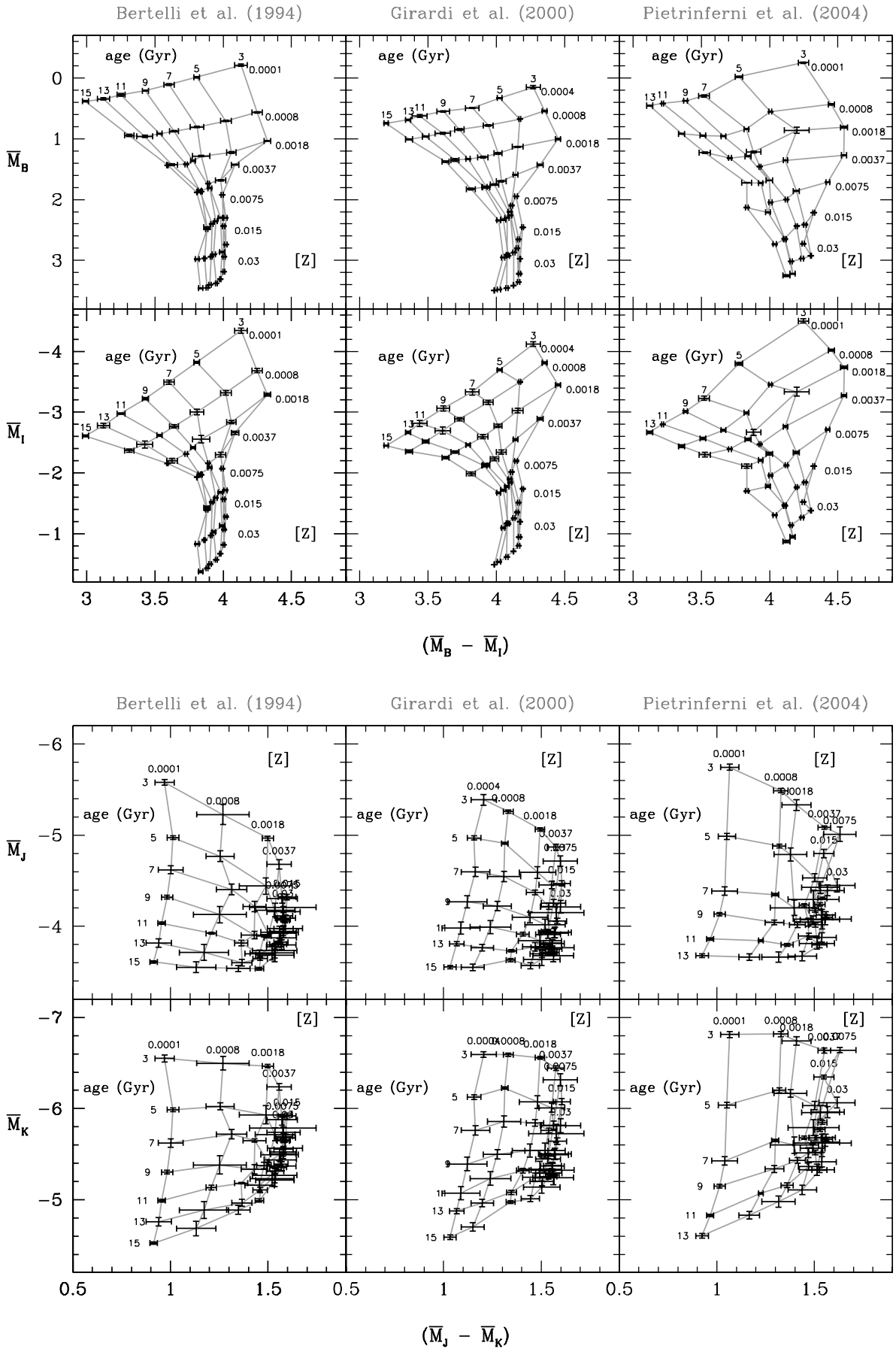

Fig. 3. FCMDs obtained using respectively the stellar evolution libraries by B94, G00 and P04. $\bar{M}_{B}$ and $\bar{M}_{I}$ have been chosen for this plot, as an example. Lines connect SSPs with same age or same metallicity. Each SSP is the result of averaging five simulations with $10^{7}$ stars. It can be seen that the three stellar evolution libraries provide comparable results, although the G00 and P04 based model results are slightly redder than those based on B94 for SSP younger than $\sim 5-7$ Gy and for SSP in the high metallicity limit, as expected after analyzing the model isochrones in Fig. 2. The age-metallicity degeneracy is broken in all cases for low metallicity $(Z \leq 0.0037)$ stellar populations.

Fig. 4. Same as Fig. 3, but here $\bar{M}_{J}$ and $\bar{M}_{K}$ have been chosen, as an example of nearIR wavelengths. Again, the three stellar evolution libraries provide comparable results, although the G00 and P04 based model results are slightly redder than those based on B94. It can be seen that the near-IR provides better age-metallicity resolution than the optical wavelengths, and the age-metallicity degeneracy is broken in all cases for low metallicity $(Z \leq 0.0018)$ stellar populations.

\subsection{Comparison with other models}

The use of SBF in stellar population synthesis has received outstanding interest in the last 15 years with works by Tonry et al. (1990), Buzzoni (1993), Worthey (1993), Ajhar \& Tonry (1994), Sodemann \& Thomsen (1996). The most recent works in this field are those by LCG00, BVA01 and C03.

LCG00 presented SBF model predictions using the latest version of the Bruzual-Charlot population synthesis models (Bruzual \& Charlot 2000), a completely updated version of the Bruzual \& Charlot (1993) models. For metallicities ranking from $1 / 200$ to 2.5 times solar, the stellar evolution is computed following the prescription of the Padova school
(Bressan et al. 1993). Their models include all the phases of the stellar evolution and the used bolometric correction was that from Lejeune et al. (1997). BVA01 used an updated version of the Vazdekis et al. (1996) code, making use of the homogeneous sets of stellar evolution models by Bertelli et al. (1994) and G00. Their theoretical models cover a wide range of ages and metallicities, and include the latest stages of the stellar evolution. They used bolometric corrections (Alonso et al. 1996, 1999) following empirical relations inferred from extensive observational photometric and spectral stellar libraries. Finally, $\mathrm{C} 03$ used the stellar evolutionary tracks from the Teramo-PiseRome group, based on the evolutionary stellar code FRANEC (Cassisi et al. 1998). Their models also cover all the stellar 
evolutionary phases. They used bolometric correction libraries from Lejeune et al. (1997) and Origlia \& Leitherer (2000). In this section, those three theoretical works are compared with our new set of IAC-star SBF theoretical calibrations.

Figure 7 shows the optical $B$-band and $I$-band FCMD obtained by BVA01, LCG00 and C03, respectively. It is clear here that the different works provide very different FCMDs. LCG00 and $\mathrm{C} 03$ results are not compatible with those from BVA01, underscoring the need for a new fully consistent SBF theoretical calibration.

Comparing Figs. 7 and 3, it can be seen that BVA01 show similar SBF trends in the FCMDs to our model predictions, the main difference being that ours predict slightly redder SBF colors. This trend is more pronounced for SSP with metallicities lower than $Z \sim 0.004$ and ages younger the $\sim 7 \mathrm{~Gy}$. These small discrepancies could be due to the use of different bolometric correction libraries. Here Castelli \& Kurucz (2003) bolometric correction library has been used, while BVA01 used bolometric corrections following empirical relations inferred from extensive observational photometric and spectral stellar libraries. On the other side, it is apparent that LCG00 and C03 results are not compatible with BVA01 and ours. In this case the observed differences don't seem to be due to differences in the used bolometric correction library, but in the stellar evolution library itself. C03 used the stellar evolutionary tracks from the Teramo-Pise-Rome group, and although LCG00 used the B94 stellar evolutionary tracks, it is worth noting that the AGB treatment is different.

In the near-IR, Fig. 8 shows the $J$-band and $K$-band FCMD obtained by BVA01, LCG00 and C03, respectively. Again, if Figs. 8 and 4 are compared, it can be seen that different works provide different fluctuation color magnitude diagrams. As in the optical wavelengths, BVA01 calibration grid results are close to ours, while those from LCG00 and C03 show completely different trends.

Figure 9 shows the $F 439 W$ and $F 814 W$ calibration grid obtained by $\mathrm{C} 03$. BVA01 and LCG00 results are not shown here because $F 439 \mathrm{~W}$ and $F 814 \mathrm{~W}$ filters were not computed in their SBF calibrations. It can be seen here that these results are similar to those obtained in the $B$-band and $I$-band diagrams.

With the aim of performing a more detailed comparison between the different SBF theoretical calibrations, the age and metallicity trends can be analyzed. In this context, Fig. 10 shows a comparison of theoretical SBF calibrations as a function of metallicity for SSP with similar ages. Our 13 Gyr SBF results, based on B94, G00 and P04, respectively, are plotted as circles with solid line, BVA01 12.59 Gyr as triangles with dotted line, LCG00 12 Gyr as squares with short dash, and C03 13 Gyr as pentagons with long dash. $\bar{M}_{U}, \bar{M}_{V}, \bar{M}_{I}$ and $\bar{M}_{K}$ have been considered in this plot. It can be clearly seen that the general trends are equivalent for all the synthetic SBF. For $U, V$ and $I$ filters, our results reside in the average of the previous works, and for the near-IR case, our SBF results are comparable to those by BVA01, slightly brighter than the rest. Figure 11, on the other side, shows the same as Fig. 10, but for the HST WFPC2 and ACS filters F439W, F555W and F814W. Here, our 13 Gyr SBF results, based on B94, G00 and P04, respectively, are plotted as circles with solid line, LCG00 12 Gyr as squares with short dash, and C03 13 Gyr as pentagons with long dash. Same trends as in Fig. 10 are found for the HST filters.

Considering solar metallicity as a standard reference for comparison, the age trends for the different SBF calibrations can be compared. With this aim, Fig. 12 shows a comparison of theoretical SBF calibrations for $Z=0.02$. Our SBF results, based on B94, G00 and P04, respectively, are plotted as circles with solid line, BVA01 as triangles with dotted line, LCG00 as squares with short dash, and $\mathrm{C} 03$ as pentagons with long dash. $\bar{M}_{U}, \bar{M}_{V}, \bar{M}_{I}$ and $\bar{M}_{K}$ have been considered in this plot. It can be seen that the general trend is the same for all SBF calibrations. Our SBF results are close to the average of the previous work's results. This is particularly true for the P04 based models.

Summarizing, Figs. 3 to 9 show that the theoretical SBF calibration presented in this work is stable and self consistent. The SBF results obtained from our three sets of models, B94, G00 and P04, show equivalent FCMDs and age and metallicity trends. Comparing with the previous SBF models predictions, it can be seen that BVA01 is the one showing a FCMD similar to ours, while LCG00 and C03 show incompatible results. Focusing on the SBF age and metallicity trends alone, Figs. 10 to 13 show that all SBF calibrations show similar age and metallicity general trends. In order to perform a more detailed test of the different SBF theoretical calibrations, model predictions will be compared with observational data in next section.

\subsection{Comparison with observational data}

At this point, no conclusion regarding which SBF calibration is better or which is worse can be reached from the previous comparison between the different SBF theoretical calibrations alone. A detailed comparison with observational data is needed to ensure the reliability and/or the limits of each one of the theoretical calibrations. In this section, the SBF theoretical calibrations are compared with observational data of galaxies, globular clusters (GCs) and the so-called superclusters. Comparison between SBF model predictions and empirical data is crucial to properly test the different theoretical SBF calibrations and estimate their current global uncertainties. One idea is to compare SBF model predictions with SBF measurements of real stellar populations with externally estimated ages and metallicities, and this is done next. This way, the present SBF theoretical calibrations can be empirically tested.

\subsubsection{Selected data sample}

Table 13 shows the considered sample of observed SBF magnitudes in galaxies, together with other galaxy properties. This table shows a selected subsample of galaxies from Table 8 in $\mathrm{C} 03$ (and references therein). Those are galaxies with a reliable independent distance estimate. Only galaxies with an age or metallicity determination, based on spectroscopic indices studies, have been considered. Column 1 shows the galaxy name. Columns 2 to 5 show the observed SBF magnitudes, when data is available, for the $V$ (Ajhar \& Tonry 1994; Tonry et al. 1990; 


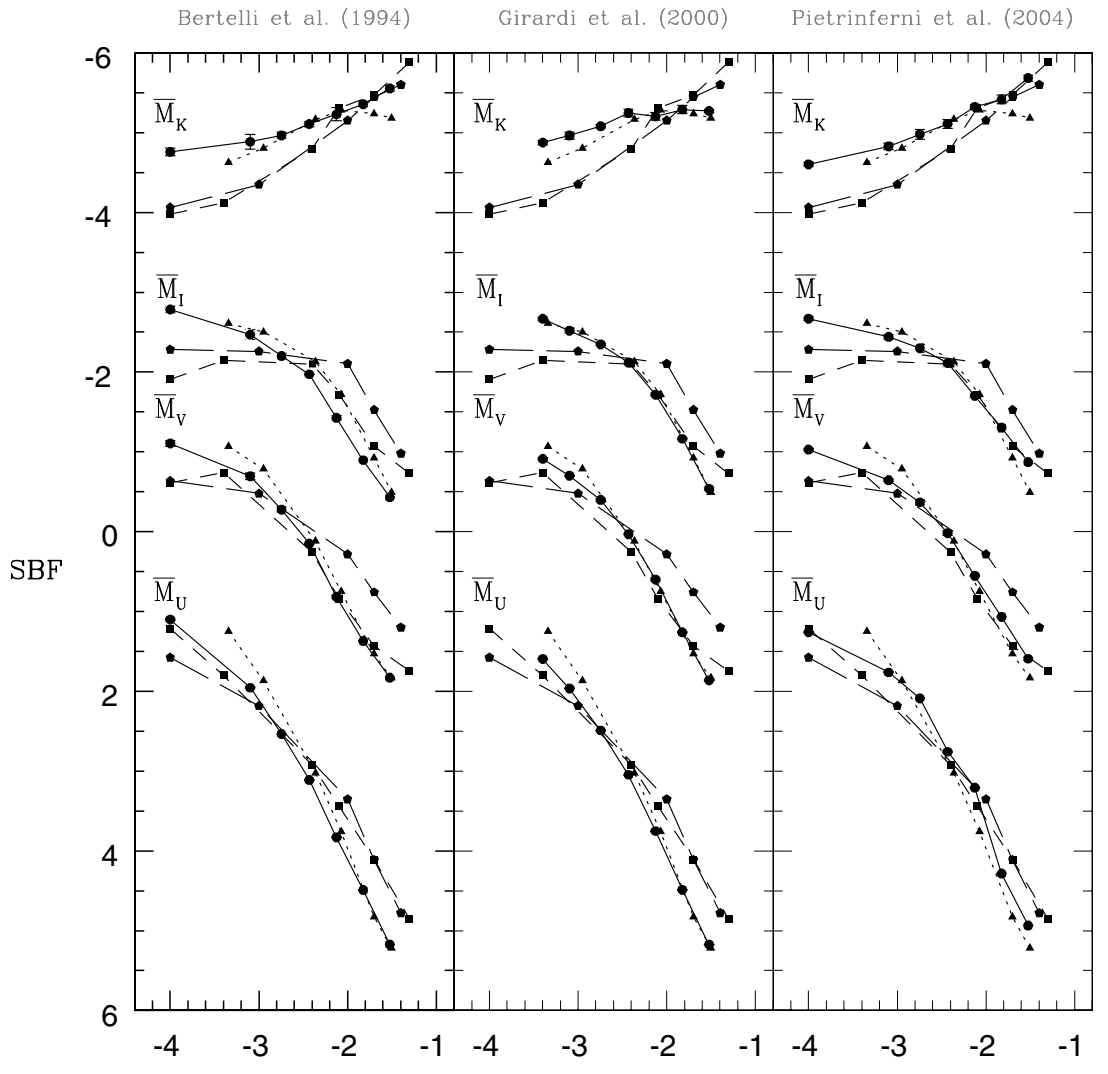

$\log (\mathrm{Z})$

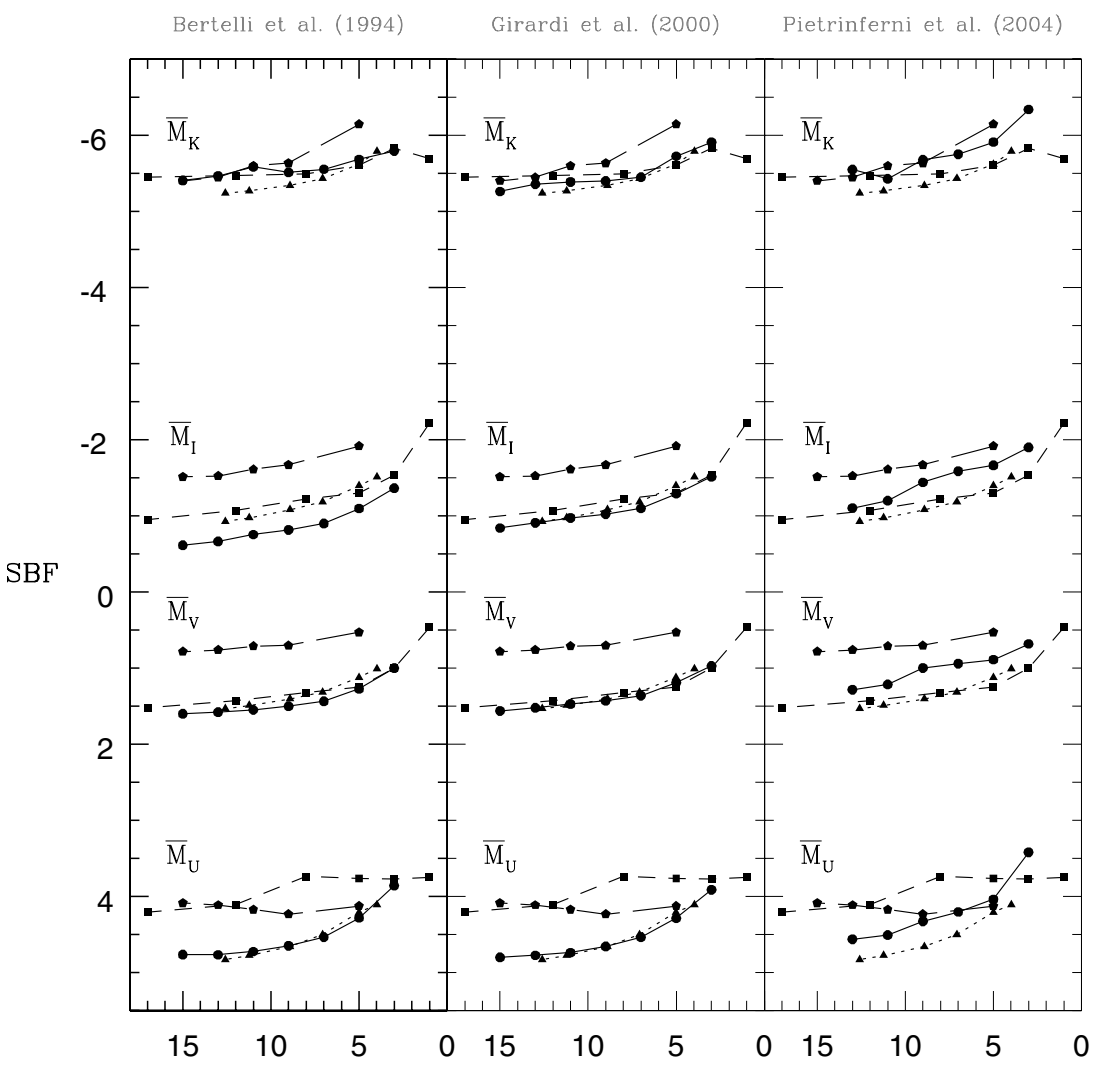

Age (Gyr)
Fig. 10. Comparison of different SBF model predictions for similar ages. Our $13 \mathrm{Gyr}$ SBF results, based on B94, G00 and P04, respectively, are plotted as circles with solid line, BVA01 $12.59 \mathrm{Gyr}$ as triangles with dotted line, LCG00 12 Gyr as squares with short dash, and C03 $13 \mathrm{Gyr}$ as pentagons with long dash. $\bar{M}_{U}, \bar{M}_{V}, \bar{M}_{I}$ and $\bar{M}_{K}$ have been considered in this plot. It can be clearly seen that the general trends are equivalent for all the synthetic SBF. For $U, V$ and $I$ filters, our results reside in the average of the previous works, and for the near-IR case, our SBF results are comparable to those by BVA01, slightly brighter than the rest.

Fig. 12. Comparison of different SBF model predictions for solar metallicity. Our SBF results, based on B94, G00 and P04, respectively, are plotted as circles with solid line, BVA01 as triangles with dotted line, LCG00 as squares with short dash, and C03 as pentagons with long dash. $\bar{M}_{U}, \bar{M}_{V}, \bar{M}_{I}$ and $\bar{M}_{K}$ have been considered in this plot. The general trend is the same for all SBF calibrations. 


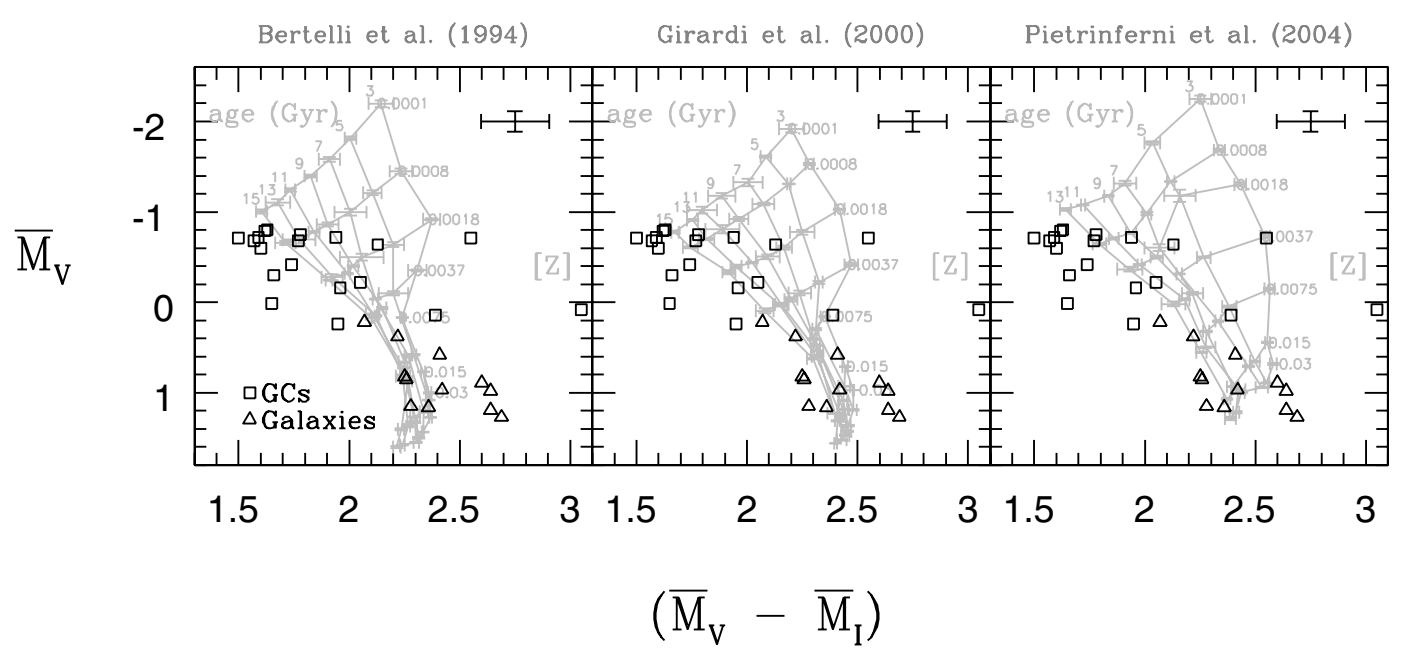

Fig. 14. SBF model predictions obtained using respectively the stellar evolution libraries by B94, G00 and P04. In order to compare our theoretical SBF results with observational data, $\bar{M}_{V}$ and $\bar{M}_{I}$ SBF magnitudes have been chosen for this plot. Open squares represent the globular clusters listed in Table 14, and open triangles the galaxies listed in Table 13. The average error bar in galaxy SBF observations is shown for reference. The precision of SBF data if high enough to provide an estimation of age and metallicity in the low metallicity region but not for high metallicities, where only metallicity estimations can be done. It can be seen that P04 based models provide a SBF calibration that best fits the observational data in the optical wavelengths.

Blakeslee et al. 2001), I (Tonry et al. 2001), $K$ (Jensen et al. 1996; Luppino \& Tonry 1993; Jensen et al. 1998) and $F 814 W$ (Ajhar et al. 1997; Pahre 1999; Neilsen \& Tsvetanov 2000) filters, respectively. Column 6 shows the $(V-I)_{0}$ color (Tonry et al. 2001), Col. 7 the distance modulus (Ferrarese et al. 2000), Col. 8 shows the metallicity and Col. 9 the age. Age and metallicity determinations come from Terlevich \& Forbes (2002).

Before presenting the GC data, it must be noted that the number of stars in a GC $\left(10^{5}-10^{6}\right)$ is much lower than the number of stars in a galaxy. This translates in intrinsic uncertainties in SBF determinations due to statistical reasons. Stochastic effects in the brightest stars are not negligible in the SBF signal. Although the SBF measurements in GC lack of precision, we will consider them only as a reference. The considered GC are shown in Table 14. Column 1 shows the GC's name, Cols. 2 and 3 the observed SBF magnitudes in the $V$ and $I$ filters, respectively. Column 4 shows the $(V-I)_{0}$ color, Col. 5 the distance modulus, and Col. 6 the metallicity. SBF and color data comes from Ajhar \& Tonry (1994), while the distance modulus and metallicity come from Harris (1996). It can be seen in this table that the error bars of the SBF measurements in GC are larger than those in galaxies, shown in Table 13, underscoring the intrinsic uncertainties of measuring SBF in GCs.

The problem with the SBF determination in GC can be solved considering the co-adding of resolved GCs. González et al. (2004) and González-Lópezlira et al. (2005) presented the first attempt to empirically calibrate SBF in the near-IR. They used the co-adding of resolved globular clusters with similar age and metallicity, called "Superclusters", to compare empirical SBF with models. Here we list their superclusters older than 3 Gyr in Table 15. Column 1 shows the name of the supercluster. Columns 2 and 3 the $K$-band SBF and the $(V-I)_{0}$ color, respectively, from González-Lópezlira et al. (2005). Columns 4 and 5 show the estimated age and metallicity, from González et al. (2004). These age and metallicity determinations, as well as the SBF data, can be considered precise enough to impose restrictions to the SBF theoretical calibrations in the near-IR.

\subsubsection{Comparison with observational data}

With the aim of comparing the different SBF theoretical calibrations with real observations, data on Tables 13 to 15 will be used to test all the theoretical SBF calibrations in the both the optical and near-IR wavelengths. Even though SBF measurements in GCs are intrinsically limited due to the stochastic behavior of the low number of brightest stars, we remark that GC data in Table 14 will be used here for reference only. No strong constrain will be imposed to the SBF calibrations from comparison with GC data. Only the well determined SBF in galaxies (optical and near-IR data) and superclusters (near-IR data) will be used to test the reliability of the different SBF model predictions.

Figure 14 shows the FCMDs obtained using respectively the stellar evolution libraries by B94, G00 and P04. In order to compare our theoretical SBF results with observational data, $\bar{M}_{V}$ and $\bar{M}_{I}$ have been chosen for this plot. Open squares represent the globular clusters listed in Table 14, and open triangles the galaxies listed in Table 13. The average error bar in the galaxy SBF magnitudes is shown for reference. As it can be seen in the figure, the precision of SBF data if high enough to provide an estimation of age and metallicity in the low metallicity region, but not for high metallicities, where only information about the metallicity can be obtained. It can be also seen that the GC SBF magnitudes lie in the low metallicity, old age region as would be expected, although the dispersion of the points is very high. Remember that GCs have been considered here just for reference. On the other hand, galaxy data lie into the high metallicity region of the diagram. Nothing can be concluded about galaxy ages because age resolution in these FCMDs is very low for high metallicities. As a remark, it can 
be seen that P04 based models provide the SBF theoretical calibration that best fits the high metallicity observational data in the optical wavelengths. This result will be reiterated latter on this paper.

In order to perform a more detailed comparison with observational data, metallicity trends for different ages will be carefully analyzed in the next set of figures for all the theoretical SBF calibrations.

Figure 15 shows a comparison of $I$-band SBF observational data with our theoretical SBF model predictions, as a function of metallicity. Our SBF results, based on B94, G00 and P04, respectively, are plotted for different ages. Open squares represent the globular clusters listed in Table 14, and open triangles the galaxies listed in Table 13. The data and the theoretical calibrations show the same general trend with metallicity. Analyzing the plot with more detail, it can be seen that the GCs fit in the low metallicity, very old age region in all cases, as expected. It must be noted that $15 \mathrm{Gyr}$ stellar populations are missing in P04 based models. An extrapolation to ages older than 13 Gyr would seem to fit all GC data. On the other hand, if we pay attention to the high metallicity region, although none of the SBF model results fit galaxy data with precision, it can be seen that P04 based models offer the best results for the optical high metallicity galaxy data.

Figure 16 shows a comparison of $I$-band SBF observational data with the previous theoretical SBF calibrations. SBF model predictions, based on BVA01, LCG00 and C03, respectively, are plotted for different ages. Open squares represent the globular clusters listed in Table 14, and open triangles the galaxies listed in Table 13. From this figure, no conclusion can be deduced about which model better fits the GC data but, on the other hand, it can be seen that the SBF calibration that best works with high metallicity data is $\mathrm{C} 03$ based models. BVA01 and LCG00 SBF calibrations are not able to reproduce galaxy data.

In the near-IR the situation is very different. Figure 17 shows a comparison of $K$-band SBF observational data with our theoretical SBF calibrations. SBF results, based on B94, G00 and P04, respectively, are plotted for different ages. Open squares represent the superclusters listed in Table 15, and open triangles the galaxies listed in Table 13. Comparing with galaxy data, the three sets of models reproduce fairly well the high metallicity SBF. If supercluster data is considered, the situation is different. Now P04 based models provide the best fit to data, offering an age estimation for the superclusters that fully agrees with the age estimations shown in Table 15. This is a big argument favoring the $\mathrm{P} 04$ based models, since this is the only SBF calibration to date that properly reproduces near-IR supercluster data, as we will see next.

Figure 18 shows a comparison of $K$-band SBF observational data with previous theoretical SBF calibrations. SBF results, based on BVA01, LCG00 and C03, respectively, are plotted for different ages. Open squares represent the Superclusters listed in Table 15, and open triangles the galaxies listed in Table 13. It can be seen here that LCG00 and C03 SBF calibrations fail to reproduce supercluster observations. These calibrations only reproduce observational data at high metallicities. On the other hand, BVA01 SBF calibration shows a similar trend as ours, but age determinations for the superclusters is less precise than those from $\mathrm{P} 04$ based models.

Finally, Fig. 19 shows the same as Fig. 15, but for the $F 814 \mathrm{~W}$ filter. Open triangles the galaxies listed in Table 13. Again, P04 based models are the only able to reproduce high metallicity galaxy data.

As a conclusion, Figs. 15 to 19 prove that the P04 theoretical SBF calibration, presented in this paper, offers the best agreement with observational data. Previous attempts to calibrate SBF in stellar populations fail in the near-IR for the low metallicity superclusters.

\subsubsection{Distance scale calibration}

A major effort has been achieved in SBF empirical calibration to measure accurate distances to nearby galaxies. Tonry et al. (2000, 2001) provide the best $I$-band SBF distance calibration based on observations of 300 early-type galaxies:

$\bar{M}_{I}=-1.74 \pm 0.008+(4.5 \pm 0.25)\left[(V-I)_{0}-1.15\right]$.

This empirical calibration can be compared with the SBF model predictions presented in this paper. Figure 20 shows $\bar{M}_{I}$ model results versus $(V-I)$ for the three stellar evolution libraries used in this work. Thick line represents Tonry et al. (2000) I-band SBF empirical calibration for old stellar populations. It is compatible with the P04 based SBF models for old populations for the full metallicity range. Agreement is also found with the Padua (B94 and G00) based SBF models for old populations, but restricted to the intermediate to low metallicity range. Galaxy and globular cluster data have been also plotted in this figure. Open squares represent the globular clusters listed in Table 14, and open triangles the galaxies listed in Table 13. The average error bar in galaxy SBF observations is shown for reference. Again, it can be seen that P04 based models provide a SBF calibration that best fits the observational data in the optical wavelengths. It is worth noting that, as it was mentioned before, globular clusters have intrinsic uncertainties in SBF determinations due to statistical reasons. Stochastic effects in the globular cluster's brightest stars are not negligible in the SBF signal. Although the SBF measurements lack of precision, globular cluster data have been considered only as a reference. For this reason, individual globular clusters are not fitted in Fig. 20. The only conclusion that can be reached from globular cluster data is that they lie in the low metallicity region of Fig. 20.

In the near-IR, Liu et al. (2002) presented an SBF calibration for distance measurements in the $K_{\mathrm{S}}$-band. They based the SBF calibration on observations of 19 early-type galaxies in the Fornax Cluster.

$\bar{M}_{K_{\mathrm{S}}}=-5.84 \pm 0.04+(3.6 \pm 0.8)\left[(V-I)_{0}-1.15\right]$.

Due to the fact that the bluest galaxies used for the empirical $K_{\mathrm{S}}$-band SBF calibration have high metallicity and are, in average, younger than $5 \mathrm{Gyr}$ (Liu et al. 2002), the calibration is only valid for $1.05<(V-I)_{0}<1.25$ and galaxies younger than 5 Gyr. Figure 21 shows $\bar{M}_{K}$ model predictions versus $(V-I)$ for the three stellar evolution libraries used in this work. Thick 


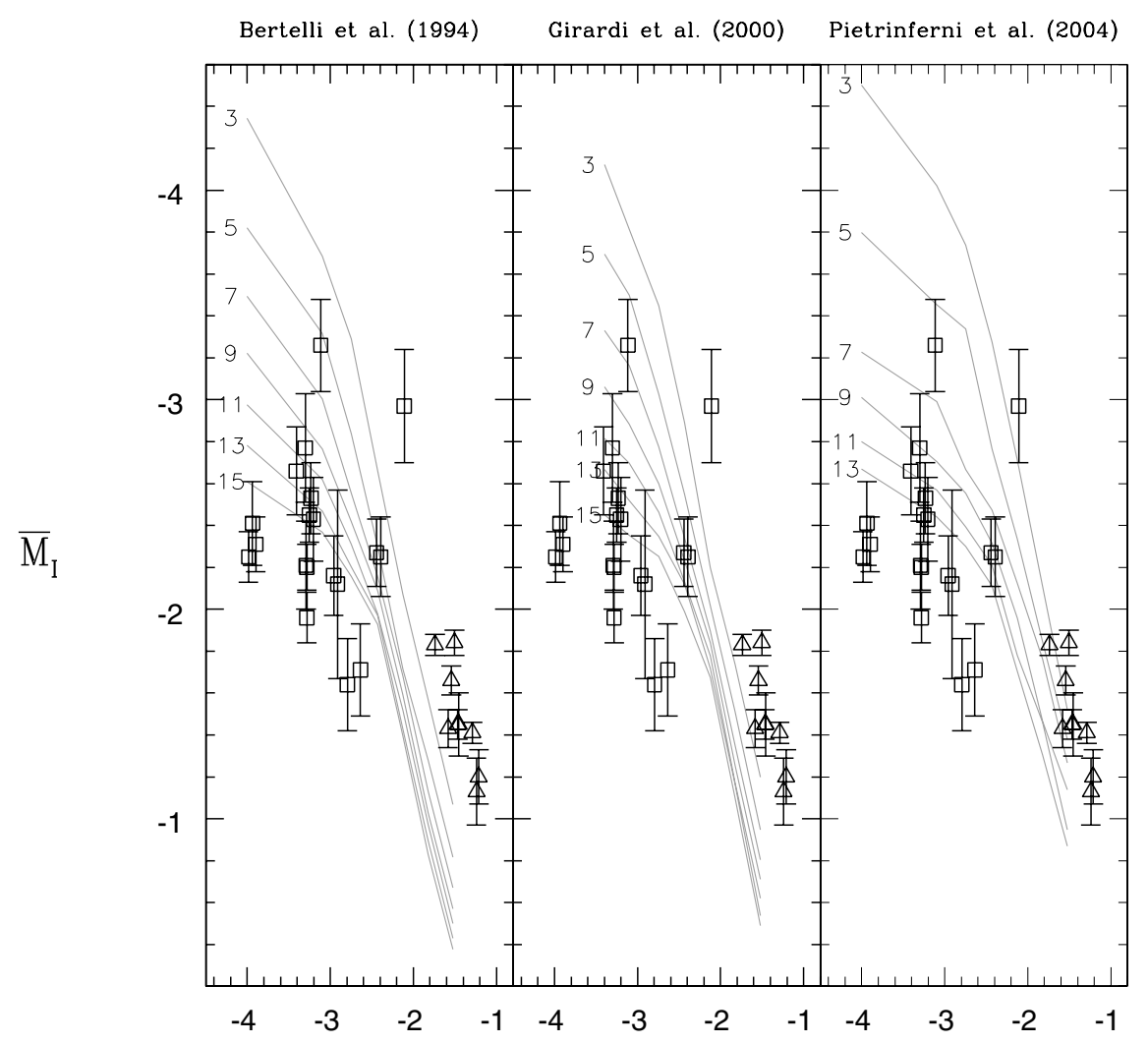

$\log (Z)$

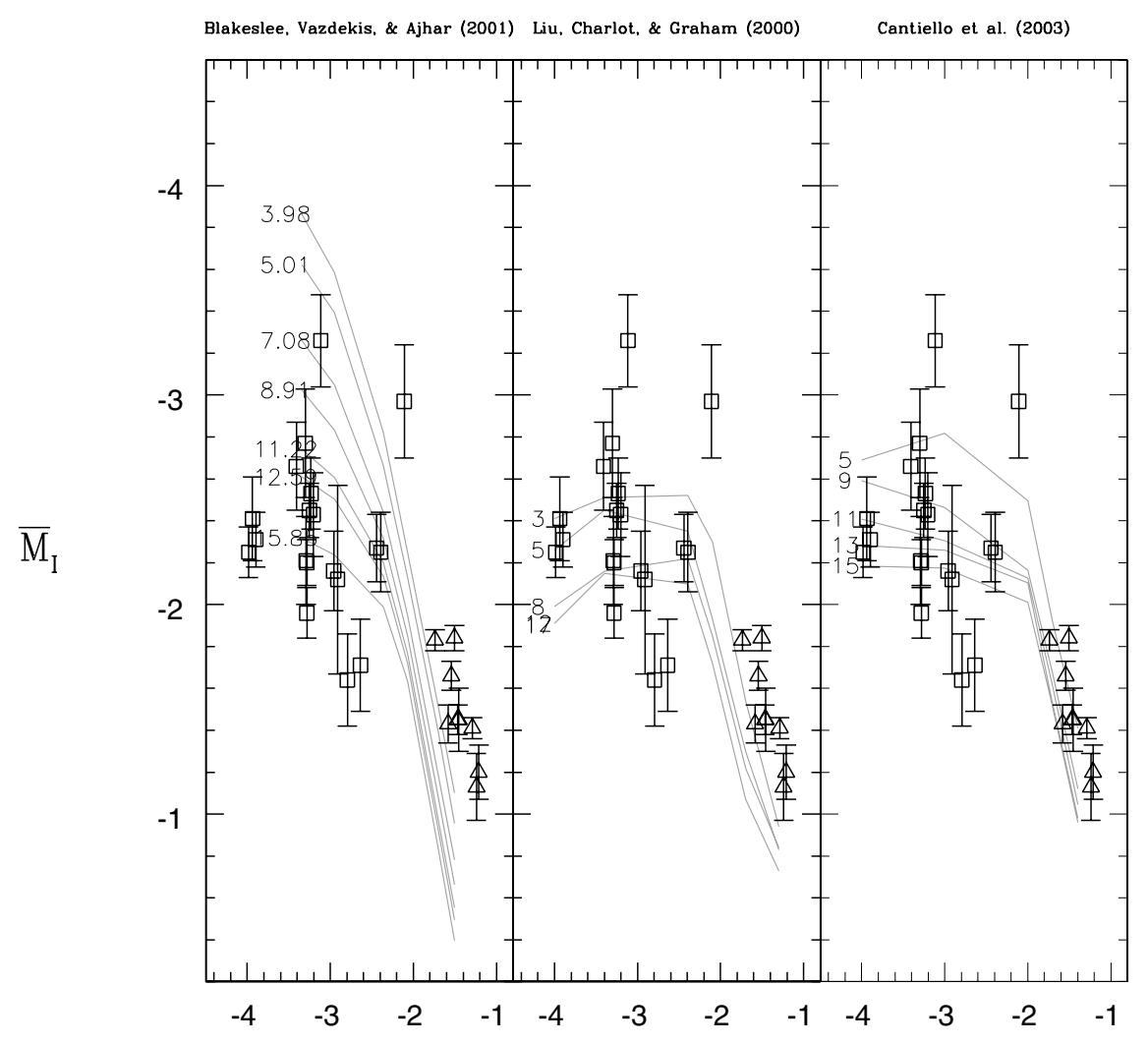

$\log (\mathrm{Z})$
Fig. 15. Comparison of $I$-band observational data with our theoretical SBF calibrations. Our SBF results, based on B94, G00 and P04, respectively, are plotted for different ages. Open squares represent the globular clusters listed in Table 14, and open triangles the galaxies listed in Table 13. The data and the theoretical calibrations show the same general trend with metallicity. Analyzing the plot with more detail, it can be seen that the globular clusters fit in the low metallicity, very old age region in all cases, as expected. An extrapolation to older than $13 \mathrm{Gyr}$ ages in the P04 based models would seem to fit all globular cluster data. On the other hand, although none of the SBF model results fit galaxy data with precision, $\mathrm{P} 04$ based models are best for reproducing high metallicity galaxy data.

Fig. 16. Comparison of I-band observational data with previous theoretical SBF calibrations. SBF results, based on BVA01, LCG00 and C03, respectively, are plotted for different ages. Open squares represent the globular clusters listed in Table 14, and open triangles the galaxies listed in Table 13. The SBF calibration that best fits data is C03 based models. The other two calibrations have problems for reproducing the high metallicity galaxy data. 


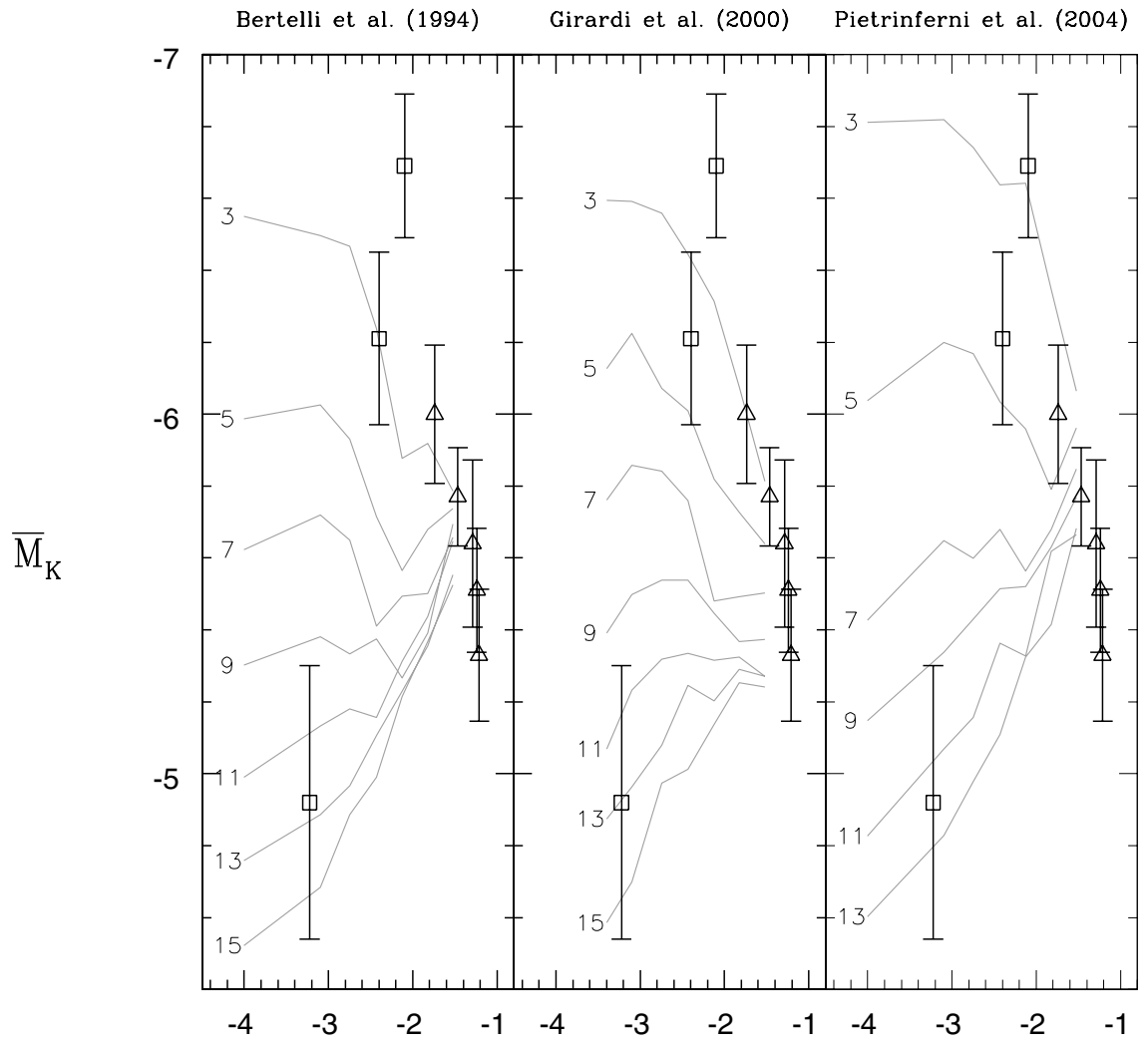

$\log (\mathrm{Z})$

Blakeslee, Vazdekis, \& Ajhar (2001) Liu, Charlot, \& Graham (2000)

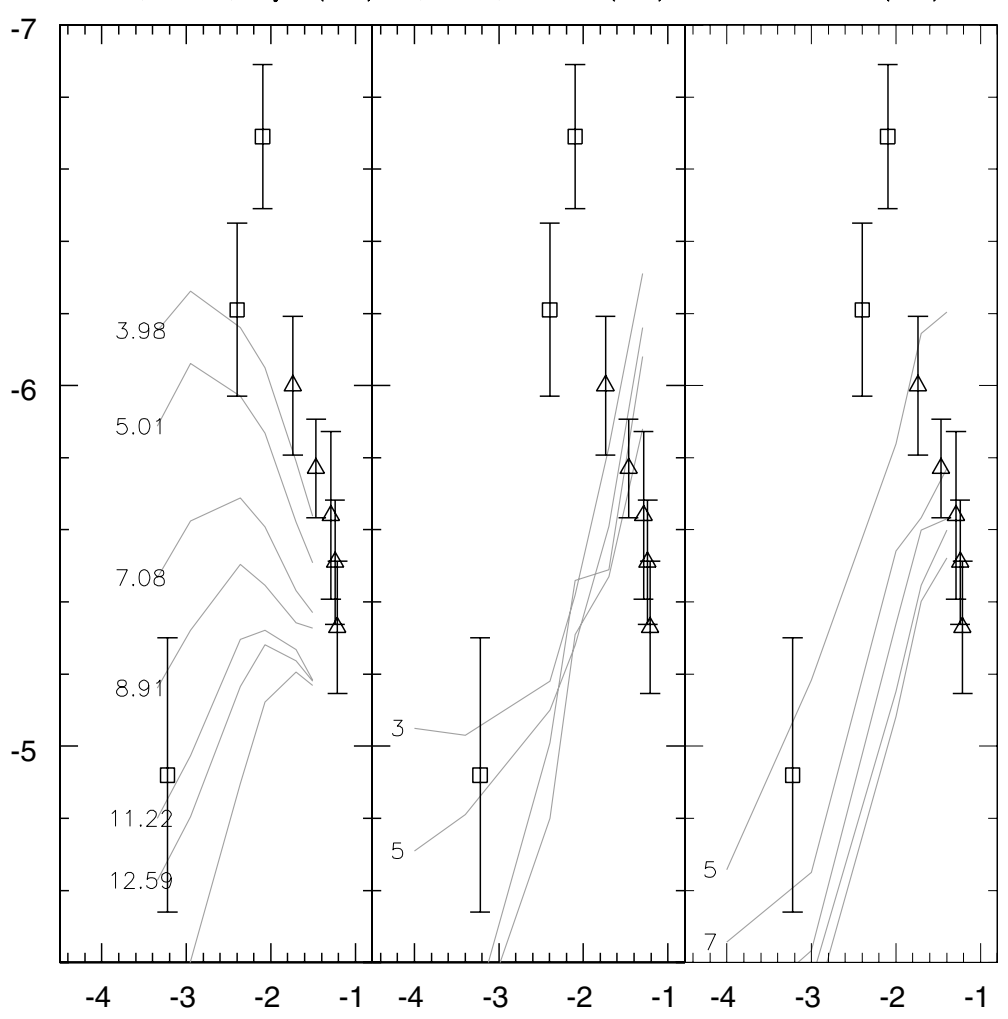

$\log (\mathrm{Z})$
Fig. 17. Comparison of near-IR ( $K$-band) observational SBF data with our theoretical SBF calibrations. Our SBF results, based on B94, G00 and $\mathrm{P} 04$, respectively, are plotted for different ages. Open squares represent the Superclusters listed in Table 15, and open triangles the galaxies listed in Table 13. Comparing with galaxy data, the three sets of models reproduce fairly well the high metallicity SBF. If we consider the Superclusters, the situation is different. Now P04 based models provide the best fit to data, offering an age estimation for the Superclusters that agree with the ages shown in Table 15 .

Fig. 18. Comparison of near-IR ( $K$-band) observational data with previous theoretical SBF calibrations. SBF results, based on BVA01, LCG00 and $\mathrm{C} 03$, respectively, are plotted for different ages. Open squares represent the Superclusters listed in Table 15, and open triangles the galaxies listed in Table 13. It can be seen here that LCG00 and C03 SBF calibrations fail to reproduce supercluster observations. These calibrations only reproduce observational data at high metallicities. On the other hand, BVA01 SBF calibration shows a similar trend as ours, but the age determinations for the superclusters is less precise than that from $\mathrm{P} 04$ based models. 


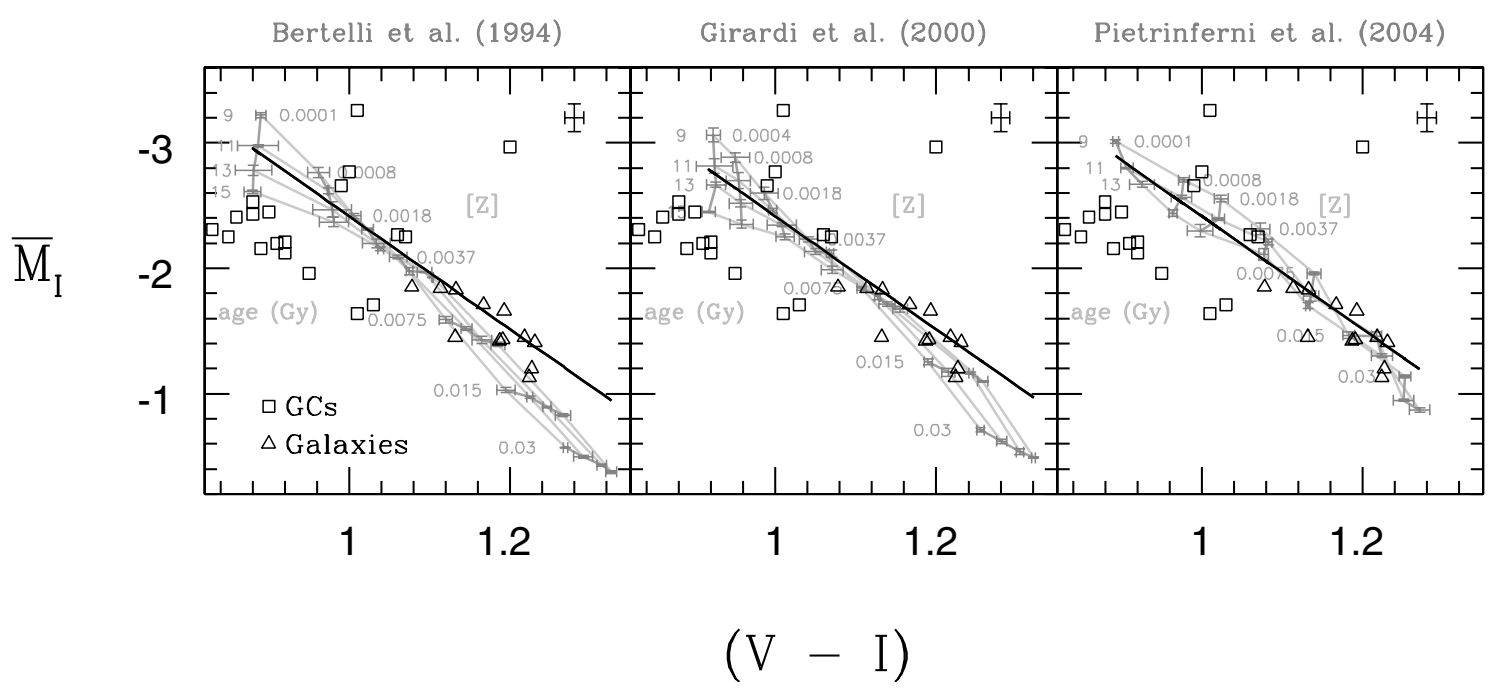

Fig. 20. SBF model predictions $\bar{M}_{I}$ versus $(V-I)$ for the three stellar evolution libraries used in this work. Thick line represents Tonry et al. (2000) $I$-band SBF empirical calibration for old stellar populations. It is compatible with the Teramo (P04) based SBF models for old populations and for the full metallicity range. Agreement is also found with the Padua (B94, G00) based SBF models for old populations, but restricted to the intermediate to low metallicity range. Galaxy and GC data have been also plotted in this figure. Open squares represent the globular clusters listed in Table 14, and open triangles the galaxies listed in Table 13. The average error bar in galaxy SBF observations is shown for reference. Again, it can be seen that P04 based models provide a SBF calibration that best fits the observational data in the optical wavelengths.

line represents Liu et al. (2002) $K_{\mathrm{S}}$-band SBF empirical calibration for stellar populations. It can be seen that our model predictions show complete agreement with Liu et al. (2002) empirical $K_{\mathrm{S}}$-band SBF calibration.

Supercluster observational data has been also plotted in this figure. Open squares represent the Superclusters listed in Table 15. Comparing this plot with the data shown in Table 15, it can be seen that our SBF calibrations are compatible with Supercluster observational data.

\subsection{4. $\bar{M}_{B}$ versus metallicity}

As an additional result, and as it can be noted in Fig. 3, age depends only weakly of $\bar{M}_{B}$ while dependence on metallicity is stronger. This is clearly seen in Fig. 22, where $\bar{M}_{B}$ versus $Z$ is represented for the three sets of SBF models B94, G00 and P04. The dependence of $\bar{M}_{B}$ on metallicity is clear in all the metallicity range, while the dependency with age is almost negligible. If only stellar populations older than $\sim 5$ Gy are considered, $\bar{M}_{B}$ can be used as a metallicity tracer, with negligible dependence on age, even for metal rich systems. We propose the use of $\bar{M}_{B}$ as a metallicity tracer for intermediate to old stellar populations.

\section{Conclusions}

We present a new set of theoretical calibrations of SBF in stellar populations both in the optical and near-IR broadband filters, as well as for the HCT WFPC2 and ACS filters. Single age, single metallicity synthetic stellar populations have been considered to perform the theoretical FCMDs. SBF analyses of unresolved stellar populations have been proved to be a very powerful technique to study and characterize the stellar content of early-type galaxies. Our model results show that SBF studies are particularly powerful for low metallicity stellar populations. In the low metallicity region, the age-metallicity degeneracy is broken, allowing an unambiguous characterization of the stellar population. The proposed approach, which has been shown to provide great possibilities to study the stellar content of low metallicity stellar populations, is more limited for studying more metal-rich galaxies. For metal-rich stellar populations, SBF has been proved to be very efficient for metallicity determinations, but the age being unresolved.

FCMDs have been computed from three different stellar evolution libraries: B94, G00 and P04. All provide comparable results, making the present theoretical SBF calibration fully consistent. Furthermore, the age-metallicity degeneracy is broken in all cases for low metallicity $(Z \leq 0.0037)$ stellar populations.

Comparing with other previous SBF theoretical calibrations, BVA01 model predictions for the FCMDs are consistent with ours, the main difference being that our models predict slightly redder SBF colors. On the other hand, LCG00 and C03 model predictions show a rather different FCMDs.

To date, the theoretical SBF calibration showing best agreement with observational data is the Teramo (P04) based models, presented in this paper. Previous works fail for reproducing the low metallicity near-IR data.

Regarding the distance scale work, both Tonry et al. (2000, 2001) empirical $I$-band and Liu et al. (2002) empirical $K$-band calibrations show full agreement with our models based on the P04 stellar evolution library. 


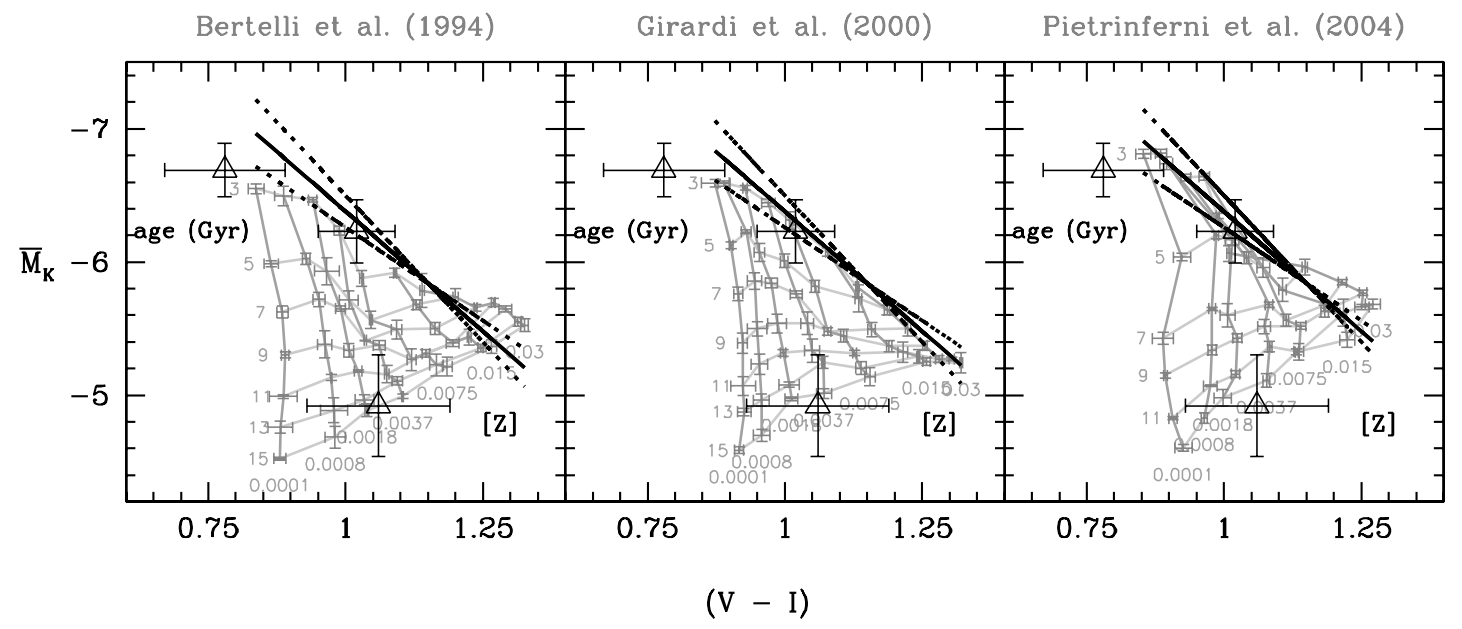

Fig. 21. SBF model predictions $\bar{M}_{K}$ versus $(V-I)$ for the three stellar evolution libraries used in this work. Thick line represents Liu et al. (2002) SBF $K$-band empirical calibration for stellar populations. This empirical $K$-SBF calibration is equivalent to our predictions for young stellar populations, but not for older ages. This is due to the fact that the early-type galaxies used for the empirical $K$-SBF calibration have high metallicity and are, in average, younger than 5 Gyr. Supercluster data has been also plotted in this figure. Open squares represent the Superclusters listed in Table 15. Comparing this plot with the data shown in Table 15, it can be seen that our SBF calibrations are compatible with Supercluster observational data.

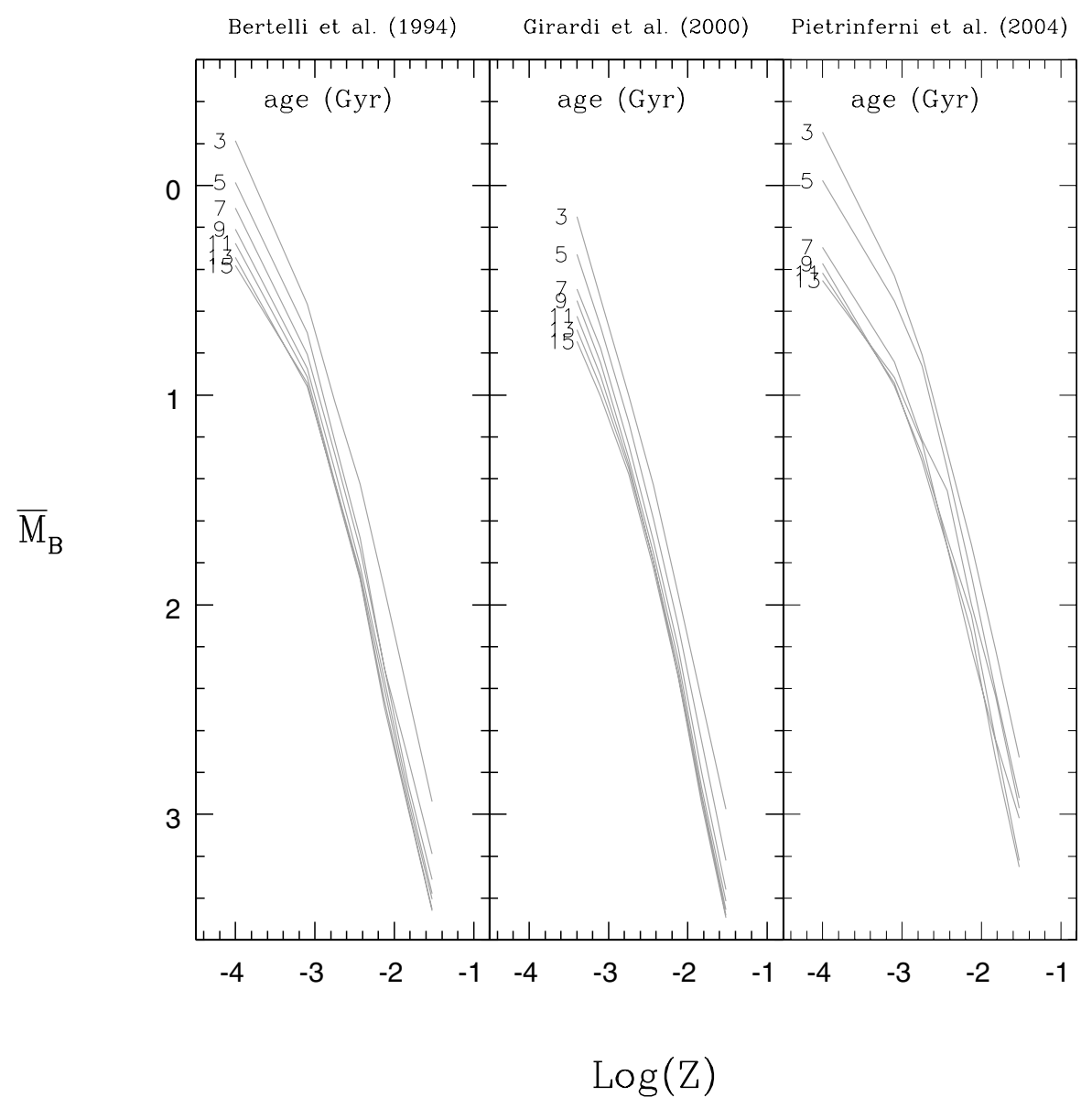

Fig. 22. $\bar{M}_{B}$ versus metallicity for the three different sets of models. A clear trend is found: $\bar{M}_{B}$ increases with metallicity. If old stellar populations (older than $\sim 5-7 \mathrm{~Gy}$ ) are considered, $\bar{M}_{B}$ can be used as a metallicity tracer.

Finally, a clear trend is found between the $B$-band SBF, $\bar{M}_{B}$, and metallicity for intermediate to old populations, so $\bar{M}_{B}$ is proposed here as a metallicity tracer.
Acknowledgements. We would like to thank the referee A. Vazdekis for his very helpful suggestions which helped to improve the final version of the paper. This work has made use of the IAC-star 
Synthetic CMD computation code. IAC-star is supported and maintained by the computer division of the Instituto de Astrofísica de Canarias. A. Marin-Franch is supported by the Gran Telescopio Canarias Postdoctoral Fellow through the University of Florida. This work has been financially supported by the the Instituto de Astrofísica de Canarias (grant P3-94) and the Education and Research Ministry of Spain (grant PNAYA-2001-1661).

\section{References}

Ajhar, E. A., \& Tonry, J. L. 1994, ApJ, 429, 557

Ajhar, E. A., Lauer, T. R., Tonry, J. L., et al. 1997, AJ, 114, 626

Alonso, A., Arribas, S., \& Martinez-Roger, C. 1996, A\&A, 313, 873

Alonso, A., Arribas, S., \& Martinez-Roger, C. 1996, A\&AS, 140, 261

Aparicio, A., \& Gallart, C. 2004, AJ, 128, 1465

Bertelli, G., Bressan, A., Chiosi, C., Fagotto, F., \& Nasi, E. 1994, A\&AS, 106, 275

Blakeslee, J. P., Vazdekis, A., \& Ajhar, E. A. 2001, MNRAS, 320, 193

Bressan, A., Fagotto, F., Bertelli, G., \& Chiosi, C. 1993, A\&AS, 100, 647

Bruzual, G. A., \& Charlot, S. 1993, ApJ, 405, 538

Bruzual, G. A., \& Charlot, S. 2000, MNRAS, 344, 1000

Buzzoni, A. 1993, A\&A, 275, 433

Cantiello, M., Raimondo, G., Brocato, E., \& Capaccioli, M. 2003, ApJ, 125, 2783

Cassisi, S., Castellani, V., degl'Innocenti, S., \& Weiss, A. 1998, A\&AS, 129, 267

Castelli, F., \& Kurucz, R. L. 2002, in Modelling of Stellar Atmospheres, ed. N. E. Piskunov et al., IAC Symp., 210, 20

Ferrarese, L., et al. 2000, ApJS, 128, 431

Gallart, C., Zoccali, M., \& Aparicio, A. 2005, ARA\&A, 43, 387
Girardi, L., Bressan, A., Chiosi, C., Bertelli, G., \& Nasi, E. 1996, A\&AS, 117, 113

Girardi, L., Bressan, A., Bertelli, G., \& Chiosi, C. 2000, A\&AS, 141, 371

González, R. A., Liu, M. C., \& Bruzual, G. A. 2004, ApJ, 611, 270

González-Lópezlira, R. A., Albarrán, M. Y., Mouhcine, M., et al. 2005, MNRAS, in press [arXiv: astro-ph/0509004]

Harris, W. E. 1996, AJ, 112, 1487

Hidalgo, S. L., Marín-Franch, A., \& Aparicio, A. 2003, AJ, 125, 1247

Jensen, J. B., Luppino, G. A., \& Tonry, J. L. 1996, ApJ, 468, 519

Jensen, J. B., Tonry, J. L., \& Luppino, G. A. 1998, ApJ, 510, 71

Jensen, J. B., Tonry, J. L., Thompson, R. I., et al. 2001, ApJ, 550, 503

Kroupa, P., Tout, C. A., \& Gilmore, G. 1993, MNRAS, 262, 545

Lejeune, T., Cuisinier, F., \& Buser, R. 1997, A\&AS, 125, 229

Liu, M. C., Charlot, S., \& Graham, J. R. 2000, ApJ, 543, 644

Liu, M. C., Graham, J. R., \& Charlot, S. 2002, ApJ, 564, 216

Luppino, G. A., \& Tonry, J. L. 1993, ApJ, 410, 81

Neilsen, E. H., \& Tsvetanov, Z. I. 2000, ApJ, 536, 255

Origlia, L., \& Leitherer, C. 2000, AJ, 119, 2018

Pahre, M. 1999, ApJ, 515, 79

Pietrinferni, A., Cassisi, S., Salaris, M., \& Castelli, F. 2004, ApJ, 612, 168

Sodemann, M., \& Thomsen, B. 1996, AJ, 111, 208

Terlevich, A. I., \& Forbes, D. A. 2002, MNRAS, 330, 547

Tonry, J. L., \& Schneider, D. P. 1988, AJ, 96, 807

Tonry, J. L., Ajhar, E. A., \& Luppino, G. A. 1990, AJ, 100, 1416

Tonry, J. L., Blakeslee, J. P., Ajhar, E. A., \& Dressler, A. 2000, ApJ, 530,625

Tonry, J. L., Dressler, A., Blakeslee, J. P., et al. 2001, ApJ, 546, 681

Vazdekis, A., Casuso, E., Peletier, R. F., \& Beckman, J. E. 1996, ApJS, 106, 307

Worthey, G. 1993, ApJ, 409, 530 (Erratum in ApJ, 418, 947) 
A. Marín-Franch and A. Aparicio: Surface-brightness fluctuations in stellar populations, Online Material p 1

\section{Online Material}


Table 1. SSP integrated colors obtained with the B94 stellar evolution library.

\begin{tabular}{|c|c|c|c|c|c|c|c|c|}
\hline$\overline{\text { Gy }}$ & $\bar{Z}$ & $\overline{(U-B)}$ & $\overline{(B-V)}$ & $\overline{(V-R)}$ & $\overline{(R-I)}$ & $(I-J)$ & $\overline{(J-H)}$ & $\overline{(H-K)}$ \\
\hline \multirow[t]{8}{*}{15} & 0.0001 & $-0.003 \pm 0.005$ & $0.607 \pm 0.006$ & $0.416 \pm 0.007$ & $0.464 \pm 0.008$ & $0.570 \pm 0.009$ & $0.503 \pm 0.011$ & $0.073 \pm 0.012$ \\
\hline & 0.0008 & $0.099 \pm 0.005$ & $0.753 \pm 0.009$ & $0.475 \pm 0.012$ & $0.506 \pm 0.014$ & $0.647 \pm 0.017$ & $0.586 \pm 0.020$ & $0.080 \pm 0.022$ \\
\hline & 0.0018 & $0.186 \pm 0.001$ & $0.826 \pm 0.001$ & $0.507 \pm 0.002$ & $0.532 \pm 0.003$ & $0.704 \pm 0.004$ & $0.645 \pm 0.005$ & $0.091 \pm 0.006$ \\
\hline & 0.0037 & $0.304 \pm 0.001$ & $0.896 \pm 0.000$ & $0.539 \pm 0.001$ & $0.565 \pm 0.000$ & $0.765 \pm 0.003$ & $0.687 \pm 0.006$ & $0.105 \pm 0.007$ \\
\hline & 0.0075 & $0.453 \pm 0.002$ & $0.964 \pm 0.003$ & $0.572 \pm 0.005$ & $0.613 \pm 0.007$ & $0.891 \pm 0.019$ & $0.734 \pm 0.028$ & $0.128 \pm 0.032$ \\
\hline & 0.0150 & $0.626 \pm 0.003$ & $1.031 \pm 0.004$ & $0.605 \pm 0.005$ & $0.661 \pm 0.008$ & $1.008 \pm 0.014$ & $0.761 \pm 0.020$ & $0.149 \pm 0.024$ \\
\hline & 0.0200 & $0.693 \pm 0.002$ & $1.055 \pm 0.002$ & $0.618 \pm 0.002$ & $0.680 \pm 0.002$ & $1.062 \pm 0.013$ & $0.774 \pm 0.020$ & $0.157 \pm 0.024$ \\
\hline & 0.0300 & $0.808 \pm 0.003$ & $1.087 \pm 0.004$ & $0.637 \pm 0.005$ & $0.689 \pm 0.005$ & $1.189 \pm 0.009$ & $0.782 \pm 0.013$ & $0.174 \pm 0.015$ \\
\hline \multirow[t]{8}{*}{13} & 0.0001 & $-0.010 \pm 0.010$ & $0.613 \pm 0.013$ & $0.417 \pm 0.015$ & $0.464 \pm 0.017$ & $0.571 \pm 0.020$ & $0.504 \pm 0.022$ & $0.072 \pm 0.023$ \\
\hline & 0.0008 & $0.090 \pm 0.007$ & $0.751 \pm 0.012$ & $0.474 \pm 0.015$ & $0.505 \pm 0.019$ & $0.651 \pm 0.025$ & $0.586 \pm 0.032$ & $0.083 \pm 0.036$ \\
\hline & 0.0018 & $0.179 \pm 0.003$ & $0.821 \pm 0.006$ & $0.504 \pm 0.010$ & $0.530 \pm 0.014$ & $0.704 \pm 0.020$ & $0.644 \pm 0.028$ & $0.090 \pm 0.031$ \\
\hline & 0.0037 & $0.285 \pm 0.003$ & $0.882 \pm 0.004$ & $0.533 \pm 0.006$ & $0.561 \pm 0.007$ & $0.770 \pm 0.009$ & $0.687 \pm 0.009$ & $0.105 \pm 0.009$ \\
\hline & 0.0075 & $0.429 \pm 0.003$ & $0.949 \pm 0.004$ & $0.563 \pm 0.006$ & $0.602 \pm 0.011$ & $0.880 \pm 0.013$ & $0.726 \pm 0.015$ & $0.128 \pm 0.017$ \\
\hline & 0.0150 & $0.592 \pm 0.002$ & $1.012 \pm 0.003$ & $0.595 \pm 0.003$ & $0.651 \pm 0.004$ & $1.000 \pm 0.010$ & $0.759 \pm 0.016$ & $0.144 \pm 0.018$ \\
\hline & 0.0200 & $0.657 \pm 0.004$ & $1.038 \pm 0.006$ & $0.609 \pm 0.008$ & $0.673 \pm 0.013$ & $1.057 \pm 0.030$ & $0.773 \pm 0.044$ & $0.157 \pm 0.049$ \\
\hline & 0.0300 & $0.776 \pm 0.001$ & $1.074 \pm 0.002$ & $0.630 \pm 0.003$ & $0.684 \pm 0.005$ & $1.172 \pm 0.008$ & $0.780 \pm 0.011$ & $0.173 \pm 0.013$ \\
\hline \multirow[t]{8}{*}{11} & 0.0001 & $-0.019 \pm 0.008$ & $0.619 \pm 0.013$ & $0.421 \pm 0.017$ & $0.466 \pm 0.019$ & $0.575 \pm 0.021$ & $0.507 \pm 0.023$ & $0.072 \pm 0.024$ \\
\hline & 0.0008 & $0.085 \pm 0.001$ & $0.745 \pm 0.001$ & $0.471 \pm 0.000$ & $0.503 \pm 0.001$ & $0.651 \pm 0.001$ & $0.592 \pm 0.001$ & $0.079 \pm 0.001$ \\
\hline & 0.0018 & $0.166 \pm 0.002$ & $0.807 \pm 0.004$ & $0.498 \pm 0.005$ & $0.525 \pm 0.005$ & $0.710 \pm 0.013$ & $0.637 \pm 0.014$ & $0.093 \pm 0.008$ \\
\hline & 0.0037 & $0.266 \pm 0.004$ & $0.866 \pm 0.004$ & $0.523 \pm 0.003$ & $0.552 \pm 0.003$ & $0.764 \pm 0.007$ & $0.681 \pm 0.014$ & $0.104 \pm 0.019$ \\
\hline & 0.0075 & $0.395 \pm 0.001$ & $0.930 \pm 0.003$ & $0.554 \pm 0.004$ & $0.592 \pm 0.006$ & $0.860 \pm 0.011$ & $0.722 \pm 0.017$ & $0.124 \pm 0.019$ \\
\hline & 0.0150 & $0.548 \pm 0.001$ & $0.990 \pm 0.002$ & $0.585 \pm 0.002$ & $0.640 \pm 0.003$ & $0.992 \pm 0.014$ & $0.757 \pm 0.021$ & $0.147 \pm 0.024$ \\
\hline & 0.0200 & $0.614 \pm 0.002$ & $1.015 \pm 0.004$ & $0.598 \pm 0.005$ & $0.661 \pm 0.005$ & $1.064 \pm 0.009$ & $0.775 \pm 0.013$ & $0.158 \pm 0.015$ \\
\hline & 0.0300 & $0.742 \pm 0.004$ & $1.061 \pm 0.006$ & $0.620 \pm 0.008$ & $0.671 \pm 0.009$ & $1.167 \pm 0.011$ & $0.781 \pm 0.012$ & $0.174 \pm 0.013$ \\
\hline \multirow[t]{8}{*}{9} & 0.0001 & $-0.021 \pm 0.005$ & $0.617 \pm 0.004$ & $0.422 \pm 0.004$ & $0.468 \pm 0.005$ & $0.581 \pm 0.006$ & $0.514 \pm 0.008$ & $0.071 \pm 0.009$ \\
\hline & 0.0008 & $0.076 \pm 0.005$ & $0.729 \pm 0.007$ & $0.464 \pm 0.009$ & $0.498 \pm 0.010$ & $0.654 \pm 0.016$ & $0.591 \pm 0.020$ & $0.084 \pm 0.022$ \\
\hline & 0.0018 & $0.152 \pm 0.002$ & $0.788 \pm 0.004$ & $0.488 \pm 0.006$ & $0.518 \pm 0.007$ & $0.697 \pm 0.008$ & $0.644 \pm 0.009$ & $0.090 \pm$ \\
\hline & 0.0037 & $0.241 \pm 0.003$ & $0.845 \pm 0.005$ & $0.515 \pm 0.007$ & $0.546 \pm 0.008$ & $0.772 \pm 0.009$ & $0.680 \pm 0.011$ & $0.105 \pm 0.011$ \\
\hline & 0.0075 & $0.353 \pm 0.003$ & $0.902 \pm 0.004$ & $0.541 \pm 0.006$ & $0.579 \pm 0.006$ & $0.842 \pm 0.015$ & $0.713 \pm 0.023$ & $0.119 \pm 0.027$ \\
\hline & 0.0150 & $0.499 \pm 0.003$ & $0.963 \pm 0.004$ & $0.570 \pm 0.006$ & $0.625 \pm 0.010$ & $0.959 \pm 0.015$ & $0.745 \pm 0.019$ & $0.142 \pm 0.021$ \\
\hline & 0.0200 & $0.566 \pm 0.002$ & $0.990 \pm 0.002$ & $0.584 \pm 0.002$ & $0.643 \pm 0.003$ & $1.027 \pm 0.009$ & $0.762 \pm 0.015$ & $0.153 \pm$ \\
\hline & 0.0300 & $0.690 \pm 0.001$ & $1.035 \pm 0.001$ & $0.608 \pm 0.002$ & $0.661 \pm 0.002$ & $1.150 \pm 0.009$ & $0.777 \pm 0.013$ & $0.172 \pm 0.014$ \\
\hline \multirow[t]{8}{*}{7} & 0.0001 & $-0.010 \pm 0.002$ & $0.604 \pm 0.003$ & $0.418 \pm 0.006$ & $0.467 \pm 0.009$ & $0.587 \pm 0.013$ & $0.521 \pm 0.016$ & $0.072 \pm 0.018$ \\
\hline & 0.0008 & $0.073 \pm 0.002$ & $0.711 \pm 0.003$ & $0.457 \pm 0.006$ & $0.494 \pm 0.009$ & $0.654 \pm 0.013$ & $0.598 \pm 0.020$ & $0.082 \pm 0.023$ \\
\hline & 0.0018 & $0.141 \pm 0.002$ & $0.768 \pm 0.002$ & $0.480 \pm 0.004$ & $0.511 \pm 0.008$ & $0.712 \pm 0.010$ & $0.631 \pm 0.013$ & $0.107 \pm$ \\
\hline & 0.0037 & $0.219 \pm 0.001$ & $0.821 \pm 0.001$ & $0.503 \pm 0.001$ & $0.533 \pm 0.002$ & $0.746 \pm 0.003$ & $0.676 \pm 0.003$ & $0.100 \pm 0.004$ \\
\hline & 0.0075 & $0.310 \pm 0.003$ & $0.869 \pm 0.005$ & $0.526 \pm 0.006$ & $0.567 \pm 0.008$ & $0.835 \pm 0.009$ & $0.712 \pm 0.011$ & $0.120 \pm 0.014$ \\
\hline & 0.0150 & $0.442 \pm 0.002$ & $0.929 \pm 0.003$ & $0.554 \pm 0.005$ & $0.609 \pm 0.006$ & $0.939 \pm 0.010$ & $0.739 \pm 0.014$ & $0.140 \pm 0.016$ \\
\hline & 0.0200 & $04 \pm 0.004$ & $0.955 \pm 0.005$ & $0.567 \pm 0.006$ & $0.627 \pm 0.008$ & $0.994 \pm 0.013$ & $0.752 \pm 0.020$ & $0.149 \pm 0.021$ \\
\hline & 0.0300 & $0.632 \pm 0.001$ & $1.007 \pm 0.002$ & $0.593 \pm 0.002$ & $0.644 \pm 0.002$ & $1.117 \pm 0.007$ & $0.770 \pm 0.011$ & $0.165 \pm 0.013$ \\
\hline \multirow[t]{8}{*}{5} & 0.0001 & $0.012 \pm 0.004$ & $0.574 \pm 0.006$ & $0.405 \pm 0.008$ & $0.459 \pm 0.010$ & $0.590 \pm 0.011$ & $0.526 \pm 0.012$ & $0.073 \pm 0.013$ \\
\hline & 0.0008 & $0.071 \pm 0.002$ & $0.675 \pm 0.003$ & $0.443 \pm 0.004$ & $0.485 \pm 0.006$ & $0.656 \pm 0.011$ & $0.597 \pm 0.014$ & $0.083 \pm 0.015$ \\
\hline & 0.0018 & $0.121 \pm 0.006$ & $0.727 \pm 0.010$ & $0.464 \pm 0.014$ & $0.502 \pm 0.017$ & $0.695 \pm 0.017$ & $0.641 \pm 0.017$ & $0.091 \pm 0.018$ \\
\hline & 0.0037 & $0.182 \pm 0.003$ & $0.776 \pm 0.005$ & $0.484 \pm 0.009$ & $0.522 \pm 0.015$ & $0.743 \pm 0.025$ & $0.672 \pm 0.036$ & $0.101 \pm 0.042$ \\
\hline & 0.0075 & $0.254 \pm 0.001$ & $0.818 \pm 0.001$ & $0.502 \pm 0.001$ & $0.544 \pm 0.002$ & $0.798 \pm 0.004$ & $0.694 \pm 0.007$ & $0.115 \pm 0.009$ \\
\hline & 0.0150 & $0.389 \pm 0.002$ & $0.894 \pm 0.003$ & $0.538 \pm 0.004$ & $0.591 \pm 0.005$ & $0.925 \pm 0.009$ & $0.734 \pm 0.014$ & $0.139 \pm 0.015$ \\
\hline & 0.0200 & $0.459 \pm 0.003$ & $0.929 \pm 0.005$ & $0.554 \pm 0.006$ & $0.613 \pm 0.008$ & $0.986 \pm 0.020$ & $0.750 \pm 0.030$ & $0.149 \pm 0.034$ \\
\hline & 0.0300 & $0.560 \pm 0.003$ & $0.971 \pm 0.004$ & $0.574 \pm 0.004$ & $0.626 \pm 0.003$ & $1.088 \pm 0.013$ & $0.761 \pm 0.021$ & $0.163 \pm 0.025$ \\
\hline \multirow[t]{8}{*}{3} & 0.0001 & $0.036 \pm 0.003$ & $0.520 \pm 0.004$ & $0.385 \pm 0.008$ & $0.452 \pm 0.012$ & $0.623 \pm 0.014$ & $0.515 \pm 0.021$ & $0.075 \pm 0.027$ \\
\hline & 0.0008 & $0.086 \pm 0.002$ & $0.619 \pm 0.005$ & $0.418 \pm 0.009$ & $0.469 \pm 0.013$ & $0.664 \pm 0.026$ & $0.600 \pm 0.034$ & $0.085 \pm 0.035$ \\
\hline & 0.0018 & $0.124 \pm 0.001$ & $0.683 \pm 0.002$ & $0.446 \pm 0.004$ & $0.494 \pm 0.006$ & $0.709 \pm 0.007$ & $0.650 \pm 0.011$ & $0.104 \pm 0.012$ \\
\hline & 0.0037 & $0.186 \pm 0.000$ & $0.747 \pm 0.001$ & $0.472 \pm 0.004$ & $0.517 \pm 0.009$ & $0.761 \pm 0.018$ & $0.681 \pm 0.026$ & $0.106 \pm 0.030$ \\
\hline & 0.0075 & $0.249 \pm 0.002$ & $0.799 \pm 0.002$ & $0.494 \pm 0.002$ & $0.535 \pm 0.002$ & $0.804 \pm 0.005$ & $0.699 \pm 0.009$ & $0.115 \pm 0.011$ \\
\hline & 0.0150 & $0.343 \pm 0.001$ & $0.856 \pm 0.001$ & $0.519 \pm 0.001$ & $0.571 \pm 0.003$ & $0.899 \pm 0.005$ & $0.727 \pm 0.007$ & $0.135 \pm 0.008$ \\
\hline & 0.0200 & $0.387 \pm 0.001$ & $0.879 \pm 0.002$ & $0.530 \pm 0.003$ & $0.585 \pm 0.004$ & $0.938 \pm 0.010$ & $0.734 \pm 0.014$ & $0.142 \pm 0.016$ \\
\hline & 0.0300 & $0.456 \pm 0.001$ & $0.908 \pm 0.002$ & $0.544 \pm 0.003$ & $0.595 \pm 0.003$ & $1.008 \pm 0.013$ & $0.735 \pm 0.020$ & $0.155 \pm 0.023$ \\
\hline
\end{tabular}




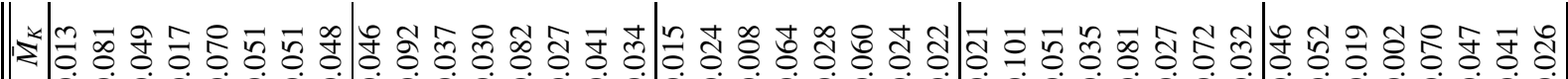

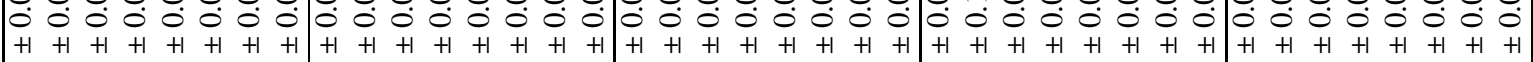

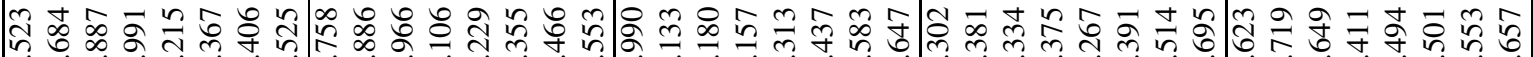

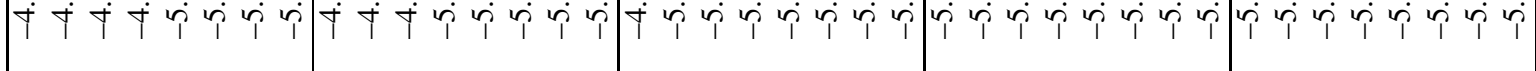

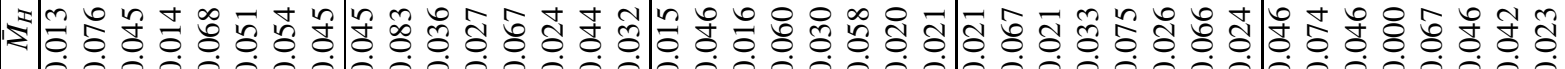

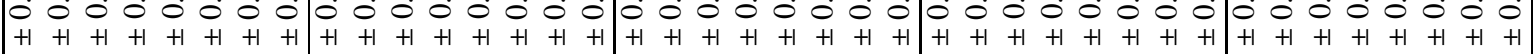

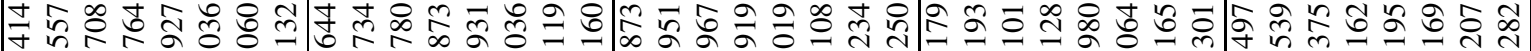
サ †

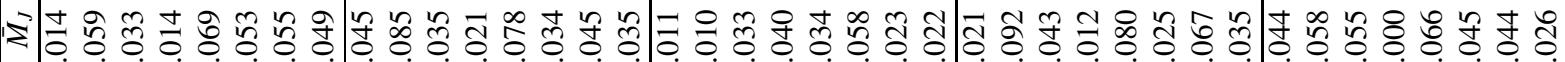

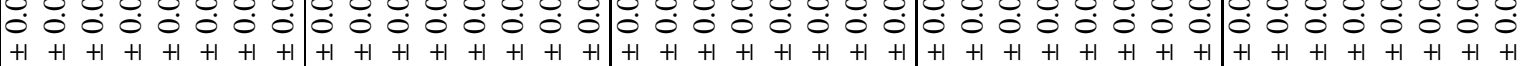

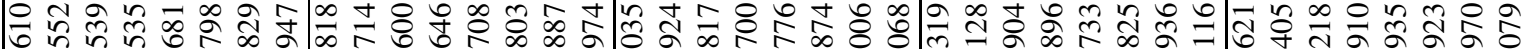

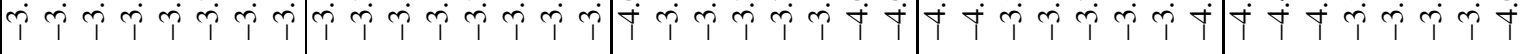

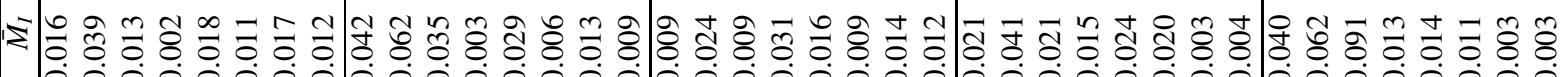

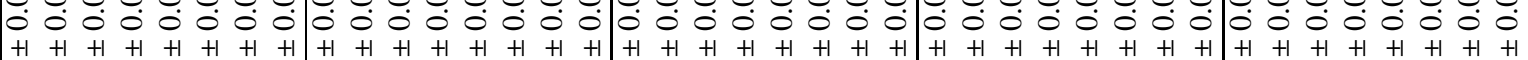

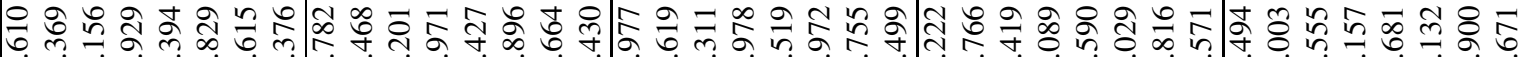
व

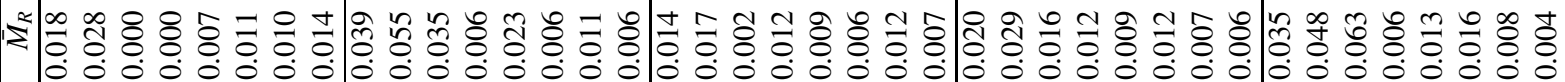
每

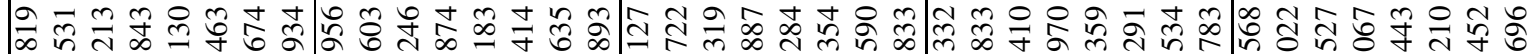
舟行

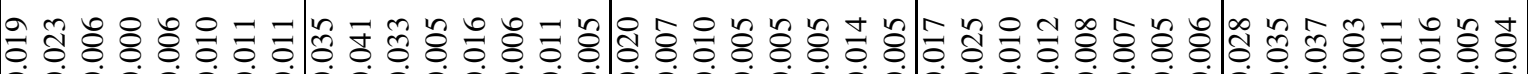
A

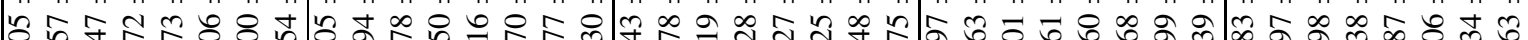
8.

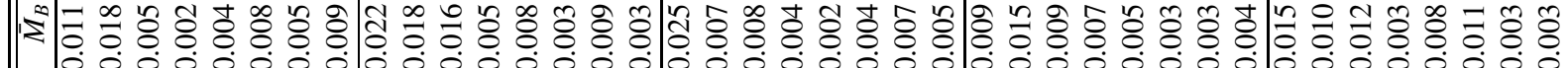
荡茾

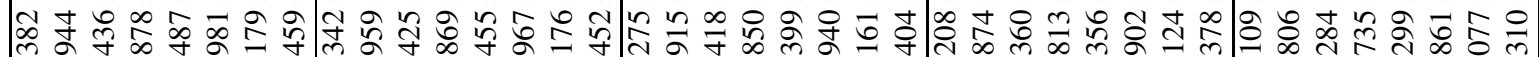

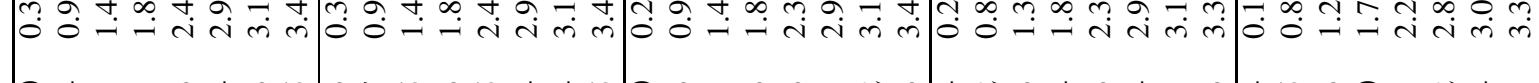

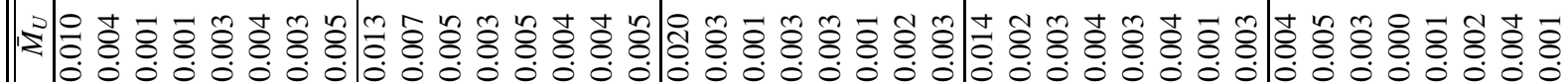

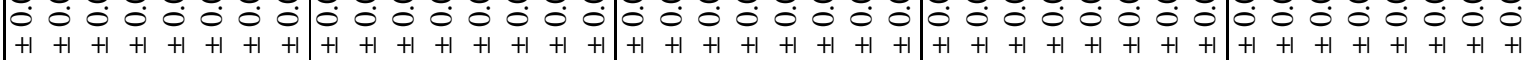

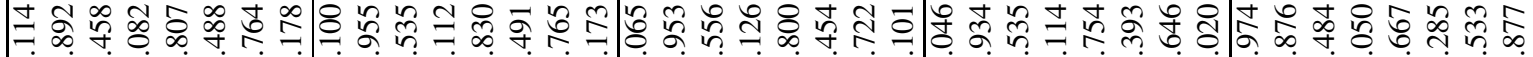
-

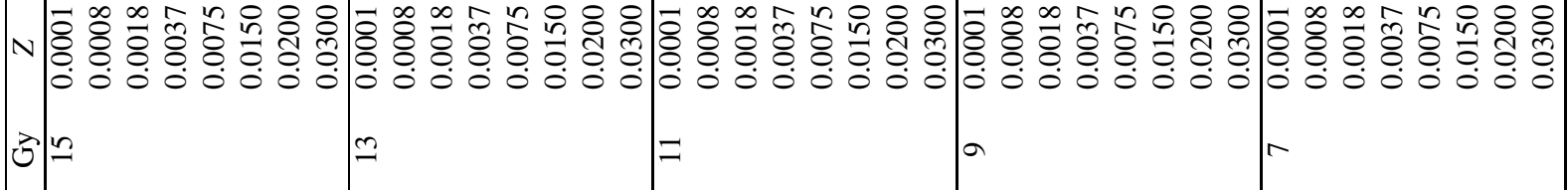




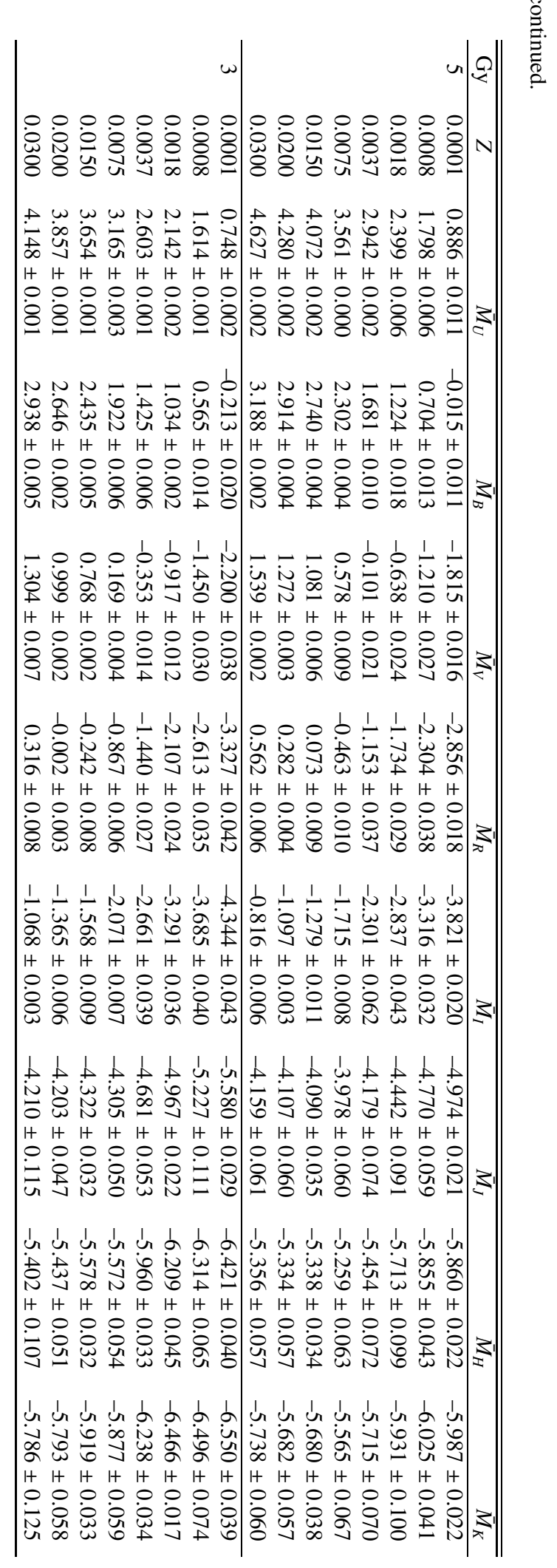


A. Marín-Franch and A. Aparicio: Surface-brightness fluctuations in stellar populations, Online Material p 5

Table 3. SSP integrated colors obtained with the G00 stellar evolution library.

\begin{tabular}{|c|c|c|c|c|c|c|c|c|}
\hline Gy & $\bar{Z}$ & $\overline{(U-B)}$ & $\overline{(B-V)}$ & $\overline{(V-R)}$ & $\overline{(R-I)}$ & $\overline{(I-J)}$ & $\overline{(J-H)}$ & $\overline{(H-K)}$ \\
\hline \multirow[t]{8}{*}{15} & 0004 & $038 \pm 0.005$ & $683 \pm 0.006$ & $441 \pm 0.006$ & $76 \pm 0.006$ & $.599 \pm 0.007$ & $.534 \pm 0.009$ & $0.073 \pm 0.010$ \\
\hline & 008 & $84 \pm 0.006$ & $739 \pm 0.008$ & $165+c$ & $193+0$ & $633 \pm 0.011$ & 013 & + \\
\hline & 0018 & $175 \pm 0.003$ & $12 \pm 0.005$ & $95 \pm 0.007$ & $17 \pm 0.009$ & 12 & $631 \pm 0.016$ & $0.087 \pm$ \\
\hline & 0037 & $.299 \pm 0.005$ & $884 \pm 0.006$ & $.526 \pm 0.008$ & $45 \pm 0.011$ & $0.745 \pm 0.017$ & $675 \pm 0.024$ & $.100 \pm 0.028$ \\
\hline & 0075 & $.444 \pm 0.004$ & $957 \pm 0.006$ & $.563 \pm 0.007$ & $52 \pm 0.006$ & $0.835 \pm 0.010$ & $0.720 \pm 0.015$ & $0.119 \pm 0.019$ \\
\hline & 150 & $626 \pm 0.003$ & $5 \pm 0$ & $607 \pm 0$ & $1 \pm 0.005$ & $0.944 \pm 0.006$ & 009 & 11 \\
\hline & 200 & $702 \pm 0.001$ & $2 \pm 0$ & 001 & $3 \pm 0.004$ & \pm 0.014 & 022 & $147 \pm$ \\
\hline & 0300 & $.836 \pm 0.001$ & $1.097 \pm 0.001$ & $0.640 \pm 0.001$ & $681 \pm 0.002$ & $1.083 \pm 0.013$ & $0.763 \pm 0.020$ & $0.155 \pm 0.023$ \\
\hline \multirow[t]{8}{*}{13} & .0004 & $032 \pm 0.004$ & $0.693 \pm 0.006$ & $0.446 \pm 0.008$ & $480 \pm 0.009$ & $0.606 \pm 0.010$ & $0.541 \pm 0.012$ & $0.073 \pm 0.012$ \\
\hline & 008 & 04 & \pm & 10 & \pm( & \pm 0 & 11 & 11 \\
\hline & 018 & \pm 0.004 & \pm 0 & 12 & $6 \pm 0$ & \pm 0 & 18 & 19 \\
\hline & 0037 & $.281 \pm 0.002$ & $874 \pm 0$ & $24 \pm 0.002$ & $46 \pm 0.002$ & $0.756 \pm 0.002$ & $683 \pm 0.005$ & $0.102 \pm$ \\
\hline & 075 & 4 & 0.9 & 06 & & 0.82 & 14 & 15 \\
\hline & 150 & \pm & \pm 0 & 02 & 22 & \pm 0 & 009 & 11 \\
\hline & & 04 & 5 & 06 & 97 & \pm 0 & 13 & 14 \\
\hline & 300 & $.796 \pm 0.002$ & 1.08 & $2 \pm 0.002$ & $72 \pm 0$ & $1.066 \pm 0.014$ & $0.757 \pm 0.021$ & $0.153 \pm$ \\
\hline \multirow[t]{8}{*}{11} & 0004 & $029 \pm 0.007$ & $0.687 \pm 0.011$ & $0.444 \pm$ & .017 & $0.609 \pm 0$ & $546 \pm 0.026$ & 028 \\
\hline & & & 7 & 09 & 12 & 0. & & 27 \\
\hline & & & & & & 06 & & \\
\hline & 037 & 003 & 6 & 09 & 10 & \pm 0 & & 017 \\
\hline & 075 & 3 & & 2 & & & & \\
\hline & 010 & 2 & 0.9 & 3 & 8 & 0 & & 45 \\
\hline & 200 & \pm & 4 & & 1 & 3 & & 16 \\
\hline & 300 & \pm & 04 & 05 & \pm & 1. & & 13 \\
\hline \multirow[t]{8}{*}{$\overline{9}$} & 0.0004 & $028 \pm 0.002$ & $0.679 \pm 0.003$ & $3 \pm 0.005$ & $0.480 \pm 0.007$ & \pm 0.010 & $0.554 \pm 0.014$ & $0.073 \pm$ \\
\hline & 008 & 4 & \pm( & & & 0.6 & & 22 \\
\hline & & & & & & 19 & & \\
\hline & & & & & & & & \\
\hline & & 1 & & 3 & 5 & 0.8 & & \\
\hline & & 1 & \pm & & & 0 & & 23 \\
\hline & & & 2 & 1 & 1 & \pm & 09 & 10 \\
\hline & 0.0300 & $0.694 \pm$ & 1.0 & 02 & $0 \pm$ & 1.04 & 018 & 019 \\
\hline \multirow[t]{8}{*}{7} & 0.0004 & $.026 \pm$ & $0.660 \pm$ & $7 \pm 0.006$ & $8 \pm$ & 0.62 & $63 \pm 0.011$ & $\overline{14}$ \\
\hline & & 5 & \pm & & & & & 09 \\
\hline & 018 & 2 & 4 & 6 & 9 & 13 & & 19 \\
\hline & & 1 & 3 & 6 & 9 & 15 & 21 & 25 \\
\hline & & \pm & \pm( & & & & & \\
\hline & & 2 & 3 & & 6 & 14 & 21 & 23 \\
\hline & & & 2 & & 3 & 3 & & \\
\hline & 300 & 2 & 1. & 03 & 94 & 1.0 & 23 & 027 \\
\hline \multirow[t]{8}{*}{5} & 004 & $0 \pm 0.002$ & $0.633 \pm 0.002$ & $8 \pm 0.002$ & $4 \pm 0$ & 0.6 & $74 \pm 0.006$ & $0.075 \pm$ \\
\hline & & & & $4 \pm 0.005$ & & & & \\
\hline & & & - & 5 & 8 & 0. & 21 & 25 \\
\hline & & 2 & 0 & 4 & 5 & 05 & 04 & 03 \\
\hline & & \pm 0 & \pm 0 & & 5 & & 05 & $17 \pm$ \\
\hline & & $7 \pm 0.002$ & $3 \pm 0$ & $4 \pm$ & $0 \pm$ & \pm 0 & 30 & 036 \\
\hline & & & 10 & & 5 & 0.9 & 19 & 23 \\
\hline & 0.0300 & 0.5 & 0.9 & 03 & 04 & $7 \pm 0$ & 16 & $0.150 \pm$ \\
\hline \multirow[t]{8}{*}{3} & 0.0004 & $0.067 \pm 0.002$ & $0.580 \pm 0.007$ & $0.408 \pm 0.014$ & $55 \pm 0.021$ & $0.626 \pm 0.018$ & $0.592 \pm 0.028$ & $0.077 \pm$ \\
\hline & 008 & $9 \pm$ & \pm & 07 & 88 & 0.6 & 12 & 0.0 \\
\hline & & & 0. & & & & & \\
\hline & & $0.160 \pm 0.004$ & $0.723 \pm 0$ & \pm 0.007 & \pm 0 & $0.753 \pm 0$ & $0.686 \pm 0.021$ & $0.109 \pm$ \\
\hline & 0.0075 & $0.223 \pm 0.001$ & $0.775 \pm 0$ & $33 \pm 0.003$ & $5 \pm 0$ & $0.825 \pm 0.007$ & $0.706 \pm 0.008$ & $0.121 \pm 0.008$ \\
\hline & & $0.323 \pm 0.002$ & $0.844 \pm 0.003$ & $14 \pm 0.003$ & $64 \pm 0.003$ & $0.871 \pm 0.008$ & $0.718 \pm 0.012$ & $0.132 \pm 0.014$ \\
\hline & & & & 03 & & $0.889 \pm 0$. & $0.721 \pm 0.012$ & $0.136 \pm 0.013$ \\
\hline & 0.0300 & $0.459 \pm 0.001$ & $0.907 \pm 0.002$ & $0.542 \pm 0.002$ & $0.590 \pm 0.002$ & $0.947 \pm 0.010$ & $0.720 \pm 0.017$ & $0.145 \pm 0.020$ \\
\hline
\end{tabular}




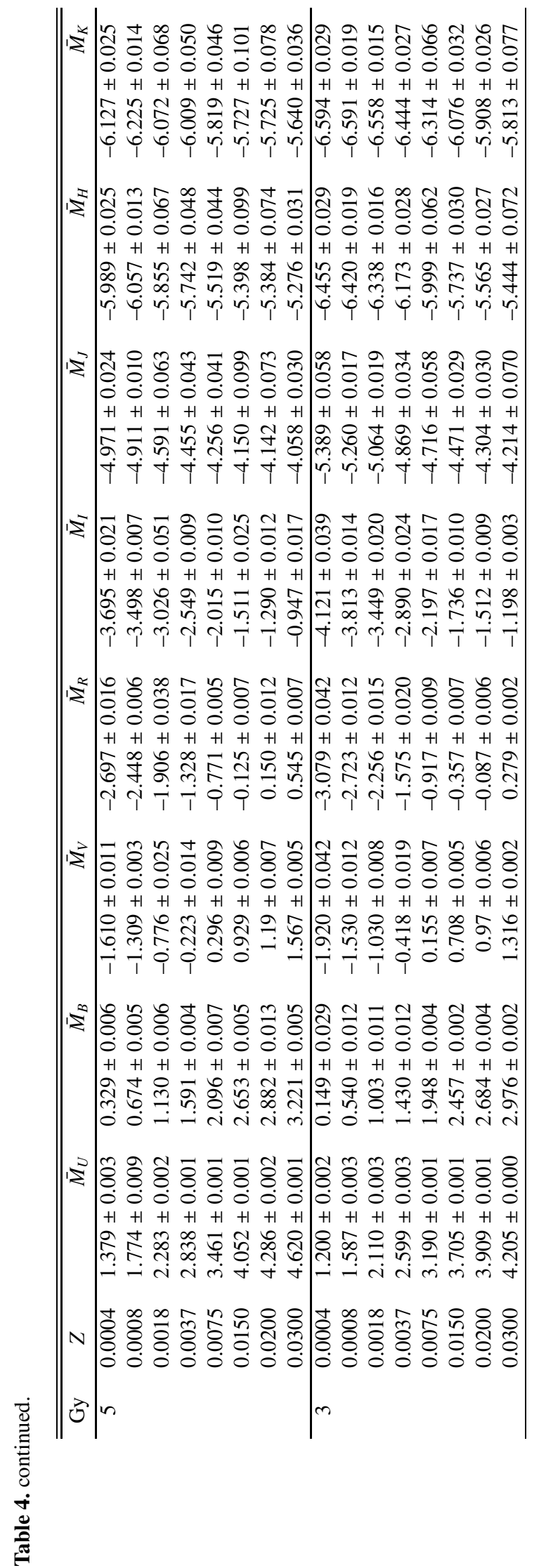


Table 5. SSP integrated colors obtained with the P04 stellar evolution library.

\begin{tabular}{|c|c|c|c|c|c|c|c|c|}
\hline$\overline{\text { Gy }}$ & $\overline{\bar{Z}}$ & $\overline{(U-B)}$ & $(B-V)$ & $\overline{(V-R)}$ & $\overline{(R-I)}$ & $\overline{(I-J)}$ & $\overline{(J-H)}$ & $\overline{(H-K)}$ \\
\hline \multirow[t]{8}{*}{13} & 0.0001 & $-0.015 \pm 0.006$ & $0.644 \pm 0.008$ & $0.440 \pm 0.010$ & $0.486 \pm 0.012$ & $0.606 \pm 0.014$ & $0.535 \pm 0.016$ & $0.077 \pm 0.017$ \\
\hline & 0.0008 & $0.113 \pm 0.007$ & $0.700 \pm 0.005$ & $0.462 \pm 0.003$ & $0.502 \pm 0.004$ & $0.667 \pm 0.006$ & $0.611 \pm 0.008$ & $0.083 \pm 0.008$ \\
\hline & 0.0018 & $0.177 \pm 0.005$ & $0.748 \pm 0.008$ & $0.481 \pm 0.010$ & $0.517 \pm 0.012$ & $0.706 \pm 0.018$ & $0.655 \pm 0.027$ & $0.091 \pm 0.031$ \\
\hline & 0.0037 & $0.263 \pm 0.003$ & $0.861 \pm 0.003$ & $0.523 \pm 0.003$ & $0.554 \pm 0.005$ & $0.759 \pm 0.011$ & $0.688 \pm 0.017$ & $0.104 \pm 0.021$ \\
\hline & 0.0075 & $0.406 \pm 0.001$ & $0.925 \pm 0.002$ & $0.547 \pm 0.002$ & $0.585 \pm 0.002$ & $0.846 \pm 0.010$ & $0.715 \pm 0.015$ & $0.121 \pm 0.018$ \\
\hline & 0.0150 & $0.568 \pm 0.004$ & $1.001 \pm 0.007$ & $0.587 \pm 0.009$ & $0.637 \pm 0.010$ & $0.956 \pm 0.014$ & $0.747 \pm 0.018$ & $0.141 \pm 0.020$ \\
\hline & 0.0200 & $0.632 \pm 0.002$ & $1.022 \pm 0.005$ & $0.595 \pm 0.008$ & $0.648 \pm 0.013$ & $1.006 \pm 0.020$ & $0.758 \pm 0.025$ & $0.149 \pm 0.028$ \\
\hline & 0.0300 & $0.730 \pm 0.003$ & $1.050 \pm 0.005$ & $0.611 \pm 0.007$ & $0.660 \pm 0.011$ & $1.117 \pm 0.014$ & $0.771 \pm 0.017$ & $0.164 \pm 0.019$ \\
\hline \multirow[t]{8}{*}{11} & 0.0001 & $-0.000 \pm 0.004$ & $0.620 \pm 0.004$ & $0.429 \pm 0.005$ & $0.478 \pm 0.006$ & $0.602 \pm 0.008$ & $0.534 \pm 0.010$ & $0.076 \pm 0.011$ \\
\hline & 0.0008 & $0.096 \pm 0.002$ & $0.729 \pm 0.005$ & $0.470 \pm 0.008$ & $0.505 \pm 0.010$ & $0.669 \pm 0.012$ & $0.614 \pm 0.013$ & $0.083 \pm 0.014$ \\
\hline & 0.0018 & $0.159 \pm 0.001$ & $0.797 \pm 0.003$ & $0.496 \pm 0.005$ & $0.525 \pm 0.006$ & $0.711 \pm 0.007$ & $0.657 \pm 0.008$ & $0.092 \pm 0.009$ \\
\hline & 0.0037 & $0.261 \pm 0.001$ & $0.864 \pm 0.001$ & $0.525 \pm 0.001$ & $0.558 \pm 0.004$ & $0.777 \pm 0.008$ & $0.699 \pm 0.013$ & $0.107 \pm 0.015$ \\
\hline & 0.0075 & $0.383 \pm 0.002$ & $0.921 \pm 0.001$ & $0.549 \pm 0.003$ & $0.587 \pm 0.009$ & $0.842 \pm 0.022$ & $0.720 \pm 0.033$ & $0.120 \pm 0.040$ \\
\hline & 0.0150 & $0.545 \pm 0.003$ & $0.990 \pm 0.004$ & $0.582 \pm 0.005$ & $0.635 \pm 0.004$ & $0.952 \pm 0.019$ & $0.744 \pm 0.039$ & $0.137 \pm 0.056$ \\
\hline & 0.0200 & $0.596 \pm 0.002$ & $1.005 \pm 0.004$ & $0.587 \pm 0.004$ & $0.641 \pm 0.004$ & $0.975 \pm 0.009$ & $0.750 \pm 0.014$ & $0.145 \pm 0.015$ \\
\hline & 0.0300 & $0.686 \pm 0.003$ & $1.030 \pm 0.005$ & $0.601 \pm 0.008$ & $0.650 \pm 0.010$ & $1.091 \pm 0.012$ & $0.764 \pm 0.015$ & $0.160 \pm 0.019$ \\
\hline \multirow[t]{8}{*}{9} & 0.0001 & $0.013 \pm 0.002$ & $0.605 \pm 0.002$ & $0.420 \pm 0.002$ & $0.473 \pm 0.003$ & $0.601 \pm 0.004$ & $0.535 \pm 0.005$ & $0.076 \pm 0.005$ \\
\hline & 0.0008 & $0.081 \pm 0.001$ & $0.733 \pm 0.002$ & $0.471 \pm 0.004$ & $0.507 \pm 0.006$ & $0.674 \pm 0.008$ & $0.621 \pm 0.010$ & $0.084 \pm 0.011$ \\
\hline & 0.0018 & $0.154 \pm 0.002$ & $0.795 \pm 0.004$ & $0.497 \pm 0.006$ & $0.527 \pm 0.006$ & $0.720 \pm 0.007$ & $0.668 \pm 0.010$ & $0.094 \pm 0.012$ \\
\hline & 0.0037 & $0.243 \pm 0.003$ & $0.851 \pm 0.005$ & $0.519 \pm 0.007$ & $0.554 \pm 0.009$ & $0.782 \pm 0.019$ & $0.698 \pm 0.025$ & $0.105 \pm 0.026$ \\
\hline & 0.0075 & $0.383 \pm 0.002$ & $0.923 \pm 0.003$ & $0.550 \pm 0.005$ & $0.590 \pm 0.007$ & $0.866 \pm 0.009$ & $0.734 \pm 0.011$ & $0.124 \pm 0.012$ \\
\hline & 0.0150 & $0.491 \pm 0.001$ & $0.960 \pm 0.002$ & $0.567 \pm 0.001$ & $0.616 \pm 0.007$ & $0.937 \pm 0.029$ & $0.742 \pm 0.046$ & $0.140 \pm 0.053$ \\
\hline & 0.0200 & $0.585 \pm 0.002$ & $1.006 \pm 0.004$ & $0.591 \pm 0.005$ & $0.651 \pm 0.007$ & $1.026 \pm 0.014$ & $0.772 \pm 0.021$ & $0.152 \pm 0.023$ \\
\hline & 0.0300 & $0.681 \pm 0.001$ & $1.033 \pm 0.003$ & $0.601 \pm 0.004$ & $0.652 \pm 0.006$ & $1.102 \pm 0.013$ & $0.770 \pm 0.018$ & $0.163 \pm 0.020$ \\
\hline \multirow[t]{8}{*}{7} & 0.0001 & $0.015 \pm 0.004$ & $0.603 \pm 0.008$ & $0.418 \pm 0.012$ & $0.471 \pm 0.015$ & $0.603 \pm 0.020$ & $0.538 \pm 0.025$ & $0.076 \pm 0.028$ \\
\hline & 0.0008 & $0.087 \pm 0.000$ & $0.730 \pm 0.002$ & $0.470 \pm 0.004$ & $0.508 \pm 0.005$ & $0.682 \pm 0.007$ & $0.630 \pm 0.010$ & $0.085 \pm 0.011$ \\
\hline & 0.0018 & $0.143 \pm 0.002$ & $0.774 \pm 0.003$ & $0.486 \pm 0.007$ & $0.520 \pm 0.012$ & $0.723 \pm 0.022$ & $0.658 \pm 0.033$ & $0.098 \pm 0.039$ \\
\hline & 0.0037 & $0.272 \pm 0.003$ & $0.864 \pm 0.004$ & $0.525 \pm 0.004$ & $0.557 \pm 0.005$ & $0.805 \pm 0.007$ & $0.709 \pm 0.008$ & $0.113 \pm 0.009$ \\
\hline & 0.0075 & $0.330 \pm 0.002$ & $0.887 \pm 0.002$ & $0.536 \pm 0.003$ & $0.577 \pm 0.004$ & $0.838 \pm 0.006$ & $0.726 \pm 0.008$ & $0.121 \pm 0.010$ \\
\hline & 0.0150 & $0.471 \pm 0.002$ & $0.951 \pm 0.002$ & $0.565 \pm 0.002$ & $0.621 \pm 0.003$ & $0.951 \pm 0.004$ & $0.754 \pm 0.005$ & $0.141 \pm 0.005$ \\
\hline & 0.0200 & $0.516 \pm 0.001$ & $0.966 \pm 0.002$ & $0.572 \pm 0.003$ & $0.635 \pm 0.003$ & $0.999 \pm 0.009$ & $0.763 \pm 0.015$ & $0.150 \pm 0.018$ \\
\hline & 0.0300 & $0.595 \pm 0.002$ & $0.989 \pm 0.002$ & $0.580 \pm 0.003$ & $0.635 \pm 0.007$ & $1.059 \pm 0.014$ & $0.759 \pm 0.019$ & $0.157 \pm 0.021$ \\
\hline \multirow[t]{8}{*}{5} & 0.0001 & $0.055 \pm 0.004$ & $0.623 \pm 0.008$ & $0.434 \pm 0.010$ & $0.489 \pm 0.012$ & $0.638 \pm 0.015$ & $0.566 \pm 0.017$ & $0.079 \pm 0.019$ \\
\hline & 0.0008 & $0.122 \pm 0.003$ & $0.727 \pm 0.003$ & $0.472 \pm 0.003$ & $0.514 \pm 0.002$ & $0.709 \pm 0.002$ & $0.650 \pm 0.000$ & $0.090 \pm 0.000$ \\
\hline & 0.0018 & $0.169 \pm 0.003$ & $0.775 \pm 0.004$ & $0.490 \pm 0.007$ & $0.525 \pm 0.010$ & $0.737 \pm 0.013$ & $0.685 \pm 0.015$ & $0.096 \pm 0.017$ \\
\hline & 0.0037 & $0.218 \pm 0.001$ & $0.806 \pm 0.002$ & $0.502 \pm 0.002$ & $0.539 \pm 0.004$ & $0.778 \pm 0.007$ & $0.707 \pm 0.011$ & $0.108 \pm 0.014$ \\
\hline & 0.0075 & $0.275 \pm 0.001$ & $0.835 \pm 0.003$ & $0.513 \pm 0.006$ & $0.558 \pm 0.010$ & $0.829 \pm 0.017$ & $0.720 \pm 0.024$ & $0.121 \pm 0.027$ \\
\hline & 0.0150 & $0.359 \pm 0.001$ & $0.873 \pm 0.002$ & $0.527 \pm 0.003$ & $0.580 \pm 0.006$ & $0.888 \pm 0.008$ & $0.718 \pm 0.015$ & $0.135 \pm 0.018$ \\
\hline & 0.0200 & $0.403 \pm 0.000$ & $0.891 \pm 0.000$ & $0.534 \pm 0.000$ & $0.590 \pm 0.001$ & $0.924 \pm 0.006$ & $0.733 \pm 0.009$ & $0.142 \pm 0.011$ \\
\hline & 0.0300 & $0.482 \pm 0.002$ & $0.921 \pm 0.003$ & $0.547 \pm 0.003$ & $0.600 \pm 0.003$ & $0.994 \pm 0.010$ & $0.734 \pm 0.017$ & $0.151 \pm 0.021$ \\
\hline \multirow[t]{8}{*}{3} & 0.0001 & $0.054 \pm 0.004$ & $0.506 \pm 0.005$ & $0.389 \pm 0.007$ & $0.464 \pm 0.012$ & $0.632 \pm 0.018$ & $0.570 \pm 0.024$ & $0.081 \pm 0.026$ \\
\hline & 0.0008 & $0.113 \pm 0.002$ & $0.580 \pm 0.004$ & $0.411 \pm 0.006$ & $0.474 \pm 0.008$ & $0.687 \pm 0.008$ & $0.646 \pm 0.009$ & $0.090 \pm 0.010$ \\
\hline & 0.0018 & $0.131 \pm 0.003$ & $0.614 \pm 0.004$ & $0.418 \pm 0.006$ & $0.477 \pm 0.010$ & $0.707 \pm 0.018$ & $0.665 \pm 0.024$ & $0.098 \pm 0.025$ \\
\hline & 0.0037 & $0.141 \pm 0.001$ & $0.649 \pm 0.003$ & $0.435 \pm 0.005$ & $0.495 \pm 0.007$ & $0.738 \pm 0.010$ & $0.691 \pm 0.013$ & $0.109 \pm 0.014$ \\
\hline & 0.0075 & $0.168 \pm 0.001$ & $0.691 \pm 0.003$ & $0.453 \pm 0.005$ & $0.511 \pm 0.008$ & $0.832 \pm 0.015$ & $0.668 \pm 0.021$ & $0.138 \pm 0.023$ \\
\hline & 0.0150 & $0.219 \pm 0.001$ & $0.736 \pm 0.002$ & $0.465 \pm 0.002$ & $0.524 \pm 0.002$ & $0.825 \pm 0.006$ & $0.689 \pm 0.007$ & $0.131 \pm 0.006$ \\
\hline & 0.0200 & $0.251 \pm 0.002$ & $0.759 \pm 0.003$ & $0.473 \pm 0.003$ & $0.534 \pm 0.005$ & $0.851 \pm 0.009$ & $0.700 \pm 0.013$ & $0.136 \pm 0.014$ \\
\hline & 0.0300 & $0.310 \pm 0.001$ & $0.787 \pm 0.002$ & $0.479 \pm 0.002$ & $0.530 \pm 0.003$ & $0.854 \pm 0.014$ & $0.677 \pm 0.023$ & $0.137 \pm 0.027$ \\
\hline
\end{tabular}




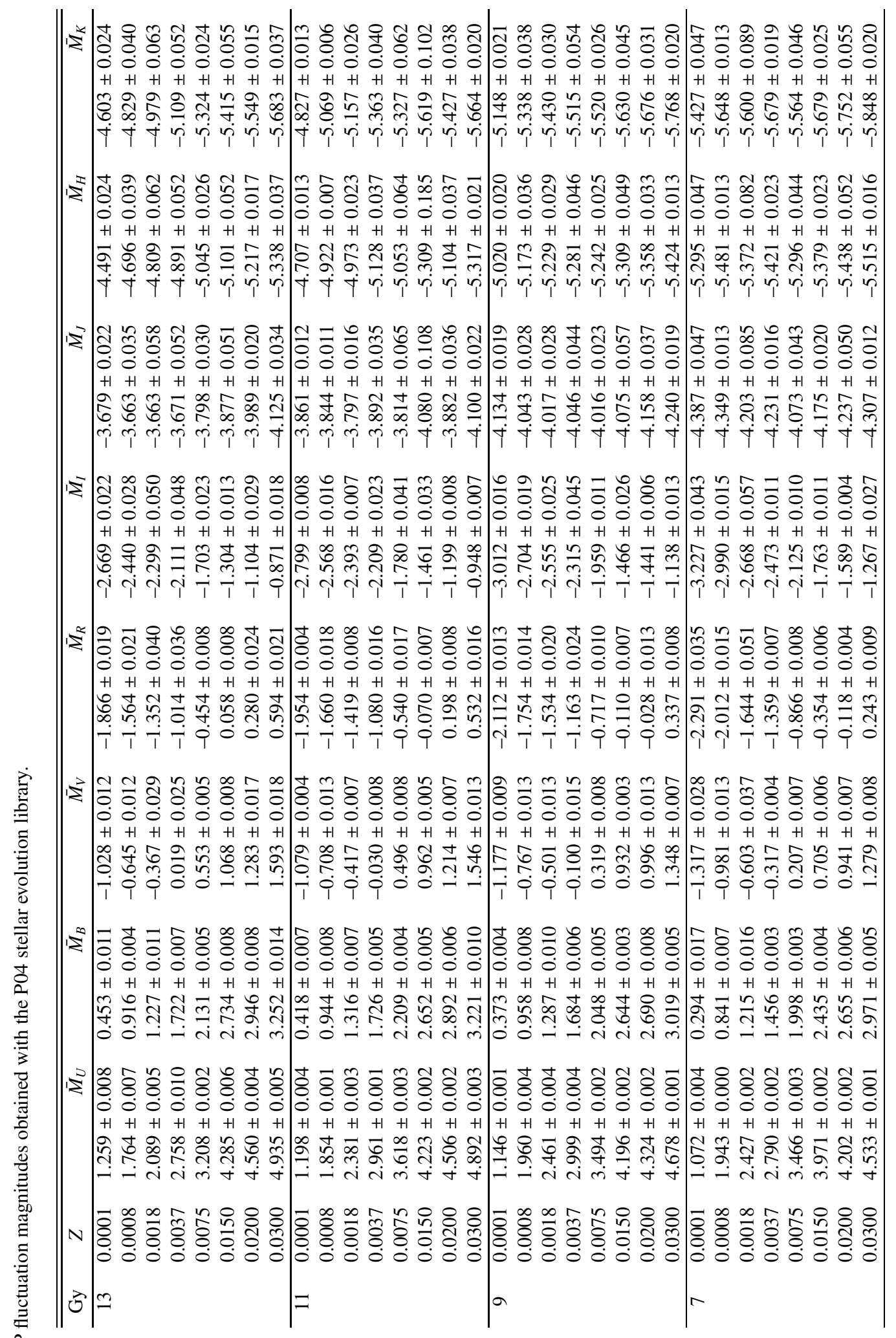




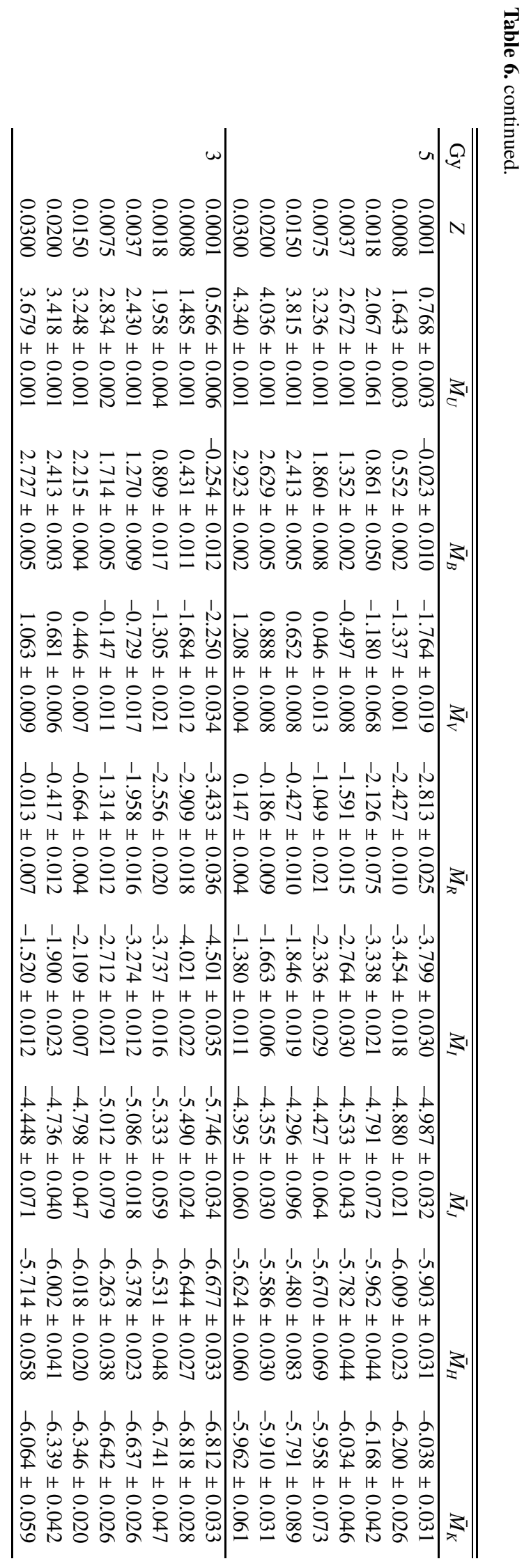


A. Marín-Franch and A. Aparicio: Surface-brightness fluctuations in stellar populations, Online Material p 11

Table 7. HST WFPC2 and ACS filters: SSP integrated colors obtained with the B94 stellar evolution library.

\begin{tabular}{|c|c|c|c|c|c|c|}
\hline Gy & $\overline{\bar{Z}}$ & $\overline{(F 218 W-F 336 W)}$ & $\bar{~}(F 336 W-F 439 W)$ & 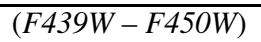 & $\overline{(F 450 W-F 555 W)}$ & $(F 555 W-F 814 W)$ \\
\hline \multirow[t]{7}{*}{15} & 0.0001 & $1.989 \pm 0.004$ & $-0.053 \pm 0.004$ & $0.204 \pm 0.005$ & $0.515 \pm 0.004$ & $0.915 \pm 0.004$ \\
\hline & 0.0008 & $2.263 \pm 0.002$ & $-0.010 \pm 0.003$ & $0.225 \pm 0.004$ & $0.561 \pm 0.005$ & $0.968 \pm 0.006$ \\
\hline & 0.0018 & $2.699 \pm 0.001$ & $0.080 \pm 0.002$ & $0.252 \pm 0.002$ & $0.621 \pm 0.003$ & $1.029 \pm 0.007$ \\
\hline & 0.0037 & $3.218 \pm 0.003$ & $0.199 \pm 0.003$ & $0.277 \pm 0.003$ & $0.678 \pm 0.003$ & $1.092 \pm 0.002$ \\
\hline & 0.0075 & $4.030 \pm 0.002$ & $0.360 \pm 0.003$ & $0.301 \pm 0.004$ & $0.738 \pm 0.005$ & $1.163 \pm 0.008$ \\
\hline & 0.0150 & $5.059 \pm 0.002$ & $0.561 \pm 0.002$ & $0.327 \pm 0.002$ & $0.800 \pm 0.002$ & $1.206 \pm 0.005$ \\
\hline & 0.0200 & $5.457 \pm 0.002$ & $0.644 \pm 0.003$ & $0.335 \pm 0.004$ & $0.822 \pm 0.004$ & $1.225 \pm 0.004$ \\
\hline \multirow[t]{7}{*}{13} & 0.0001 & $2.015 \pm 0.003$ & $-0.073 \pm 0.003$ & $0.206 \pm 0.005$ & $0.518 \pm 0.006$ & $0.921 \pm 0.006$ \\
\hline & 0.0008 & $2.245 \pm 0.002$ & $-0.023 \pm 0.004$ & $0.226 \pm 0.005$ & $0.562 \pm 0.005$ & $0.969 \pm 0.004$ \\
\hline & 0.0018 & $2.599 \pm 0.001$ & $0.066 \pm 0.003$ & $0.250 \pm 0.005$ & $0.614 \pm 0.007$ & $1.018 \pm 0.010$ \\
\hline & 0.0037 & $3.069 \pm 0.002$ & $0.171 \pm 0.004$ & $0.271 \pm 0.005$ & $0.666 \pm 0.005$ & $1.079 \pm 0.006$ \\
\hline & 0.0075 & $3.841 \pm 0.001$ & $0.330 \pm 0.003$ & $0.297 \pm 0.004$ & $0.726 \pm 0.005$ & $1.144 \pm 0.007$ \\
\hline & 0.0150 & $4.825 \pm 0.001$ & $0.519 \pm 0.001$ & $0.321 \pm 0.002$ & $0.785 \pm 0.003$ & $1.194 \pm 0.009$ \\
\hline & 0.0200 & $5.239 \pm 0.001$ & $0.602 \pm 0.001$ & $0.329 \pm 0.001$ & $0.809 \pm 0.002$ & $1.208 \pm 0.005$ \\
\hline \multirow[t]{7}{*}{11} & 0.0001 & $1.950 \pm 0.002$ & $-0.082 \pm 0.003$ & $0.203 \pm 0.004$ & $0.513 \pm 0.005$ & $0.919 \pm 0.009$ \\
\hline & 0.0008 & $2.146 \pm 0.002$ & $-0.033 \pm 0.005$ & $0.224 \pm 0.008$ & $0.558 \pm 0.009$ & $0.966 \pm 0.010$ \\
\hline & 0.0018 & $2.463 \pm 0.001$ & $0.045 \pm 0.003$ & $0.246 \pm 0.003$ & $0.608 \pm 0.004$ & $1.017 \pm 0.007$ \\
\hline & 0.0037 & $2.908 \pm 0.002$ & $0.147 \pm 0.003$ & $0.266 \pm 0.004$ & $0.653 \pm 0.006$ & $1.063 \pm 0.010$ \\
\hline & 0.0075 & $3.615 \pm 0.001$ & $0.287 \pm 0.002$ & $0.289 \pm 0.004$ & $0.709 \pm 0.006$ & $1.125 \pm 0.012$ \\
\hline & 0.0150 & $4.549 \pm 0.001$ & $0.463 \pm 0.000$ & $0.312 \pm 0.001$ & $0.767 \pm 0.003$ & $1.171 \pm 0.007$ \\
\hline & 0.0200 & $4.969 \pm 0.001$ & $0.542 \pm 0.001$ & $0.320 \pm 0.001$ & $0.791 \pm 0.001$ & $1.193 \pm 0.002$ \\
\hline \multirow[t]{7}{*}{9} & 0.0001 & $1.839 \pm 0.002$ & $-0.084 \pm 0.004$ & $0.199 \pm 0.006$ & $0.507 \pm 0.006$ & $0.915 \pm 0.008$ \\
\hline & 0.0008 & $2.016 \pm 0.003$ & $-0.042 \pm 0.003$ & $0.219 \pm 0.004$ & $0.547 \pm 0.005$ & $0.956 \pm 0.008$ \\
\hline & 0.0018 & $2.300 \pm 0.001$ & $0.028 \pm 0.003$ & $0.240 \pm 0.005$ & $0.595 \pm 0.008$ & $1.001 \pm 0.012$ \\
\hline & 0.0037 & $2.721 \pm 0.001$ & $0.118 \pm 0.001$ & $0.259 \pm 0.001$ & $0.639 \pm 0.002$ & $1.048 \pm 0.002$ \\
\hline & 0.0075 & $3.360 \pm 0.001$ & $0.240 \pm 0.002$ & $0.279 \pm 0.002$ & $0.687 \pm 0.002$ & $1.098 \pm 0.007$ \\
\hline & 0.0150 & $4.255 \pm 0.001$ & $0.407 \pm 0.001$ & $0.302 \pm 0.001$ & $0.746 \pm 0.001$ & $1.149 \pm 0.004$ \\
\hline & 0.0200 & $4.678 \pm 0.002$ & $0.485 \pm 0.002$ & $0.311 \pm 0.002$ & $0.770 \pm 0.002$ & $1.167 \pm 0.006$ \\
\hline \multirow[t]{7}{*}{7} & 0.0001 & $1.679 \pm 0.003$ & $-0.083 \pm 0.005$ & $0.194 \pm 0.006$ & $0.498 \pm 0.006$ & $0.913 \pm 0.006$ \\
\hline & 0.0008 & $1.839 \pm 0.000$ & $-0.045 \pm 0.000$ & $0.212 \pm 0.000$ & $0.535 \pm 0.000$ & $0.951 \pm 0.000$ \\
\hline & 0.0018 & $2.105 \pm 0.001$ & $0.012 \pm 0.002$ & $0.231 \pm 0.002$ & $0.578 \pm 0.003$ & $0.985 \pm 0.007$ \\
\hline & 0.0037 & $2.511 \pm 0.002$ & $0.088 \pm 0.002$ & $0.250 \pm 0.003$ & $0.622 \pm 0.003$ & $1.032 \pm 0.004$ \\
\hline & 0.0075 & $3.076 \pm 0.002$ & $0.191 \pm 0.002$ & $0.268 \pm 0.002$ & $0.667 \pm 0.004$ & $1.088 \pm 0.006$ \\
\hline & 0.0150 & $3.925 \pm 0.001$ & $0.345 \pm 0.002$ & $0.290 \pm 0.002$ & $0.722 \pm 0.002$ & $1.125 \pm 0.002$ \\
\hline & 0.0200 & $4.333 \pm 0.002$ & $0.411 \pm 0.002$ & $0.298 \pm 0.002$ & $0.742 \pm 0.002$ & $1.139 \pm 0.005$ \\
\hline \multirow[t]{7}{*}{5} & 0.0001 & $1.445 \pm 0.001$ & $-0.060 \pm 0.002$ & $0.182 \pm 0.003$ & $0.477 \pm 0.004$ & $0.895 \pm 0.005$ \\
\hline & 0.0008 & $1.590 \pm 0.001$ & $-0.045 \pm 0.003$ & $0.196 \pm 0.005$ & $0.505 \pm 0.010$ & $0.917 \pm 0.017$ \\
\hline & 0.0018 & $1.830 \pm 0.003$ & $-0.008 \pm 0.006$ & $0.215 \pm 0.008$ & $0.546 \pm 0.011$ & $0.950 \pm 0.014$ \\
\hline & 0.0037 & $2.216 \pm 0.001$ & $0.049 \pm 0.001$ & $0.233 \pm 0.002$ & $0.590 \pm 0.003$ & $1.005 \pm 0.007$ \\
\hline & 0.0075 & $2.735 \pm 0.002$ & $0.125 \pm 0.002$ & $0.247 \pm 0.001$ & $0.625 \pm 0.001$ & $1.033 \pm 0.002$ \\
\hline & 0.0150 & $3.552 \pm 0.001$ & $0.282 \pm 0.001$ & $0.276 \pm 0.001$ & $7.531 \pm 0.006$ & $1.102 \pm 0.008$ \\
\hline & 0.0200 & $3.961 \pm 0.001$ & $0.362 \pm 0.002$ & $0.289 \pm 0.003$ & $0.722 \pm 0.004$ & $1.117 \pm 0.005$ \\
\hline \multirow[t]{7}{*}{3} & 0.0001 & $1.107 \pm 0.002$ & $-0.014 \pm 0.002$ & $0.162 \pm 0.002$ & $0.444 \pm 0.006$ & $0.868 \pm 0.016$ \\
\hline & 0.0008 & $1.108 \pm 0.000$ & $-0.009 \pm 0.000$ & $0.166 \pm 0.000$ & $0.457 \pm 0.000$ & $0.895 \pm 0.000$ \\
\hline & 0.0018 & $1.507 \pm 0.001$ & $0.011 \pm 0.003$ & $0.197 \pm 0.005$ & $0.515 \pm 0.006$ & $0.932 \pm 0.010$ \\
\hline & 0.0037 & $1.874 \pm 0.002$ & $0.065 \pm 0.002$ & $0.222 \pm 0.002$ & $0.571 \pm 0.003$ & $0.987 \pm 0.003$ \\
\hline & 0.0075 & $2.353 \pm 0.002$ & $0.122 \pm 0.001$ & $0.240 \pm 0.001$ & $0.612 \pm 0.001$ & $1.039 \pm 0.009$ \\
\hline & 0.0150 & $3.042 \pm 0.002$ & $0.232 \pm 0.003$ & $0.261 \pm 0.003$ & $0.662 \pm 0.004$ & $1.061 \pm 0.005$ \\
\hline & 0.0200 & $3.383 \pm 0.002$ & $0.285 \pm 0.001$ & $0.270 \pm 0.001$ & $0.684 \pm 0.002$ & $1.075 \pm 0.004$ \\
\hline
\end{tabular}


A. Marín-Franch and A. Aparicio: Surface-brightness fluctuations in stellar populations, Online Material p 12

Table 8. HST WFPC2 and ACS filters: SSP fluctuation magnitudes obtained with the B94 stellar evolution library.

\begin{tabular}{|c|c|c|c|c|c|c|c|}
\hline Gy & $Z$ & $\bar{M}_{F 218 W}$ & $\bar{M}_{F 336 W}$ & $\bar{M}_{F 439 W}$ & $\bar{M}_{F 450 W}$ & $\bar{M}_{F 555 W}$ & $\bar{M}_{F 814 W}$ \\
\hline \multirow[t]{7}{*}{15} & 0.0001 & $3.952 \pm 0.003$ & $1.777 \pm 0.003$ & $0.772 \pm 0.012$ & $0.284 \pm 0.020$ & $-0.827 \pm 0.038$ & $-2.432 \pm 0.061$ \\
\hline & 0.0008 & $4.771 \pm 0.005$ & $2.174 \pm 0.003$ & $1.206 \pm 0.007$ & $0.671 \pm 0.009$ & $-0.537 \pm 0.015$ & $-2.282 \pm 0.024$ \\
\hline & 0.0018 & $6.277 \pm 0.003$ & $2.697 \pm 0.002$ & $1.694 \pm 0.006$ & $1.125 \pm 0.010$ & $-0.171 \pm 0.019$ & $-2.084 \pm 0.034$ \\
\hline & 0.0037 & $7.518 \pm 0.001$ & $3.321 \pm 0.005$ & $2.157 \pm 0.002$ & $1.575 \pm 0.002$ & $0.227 \pm 0.009$ & $-1.861 \pm 0.024$ \\
\hline & 0.0075 & $8.766 \pm 0.000$ & $4.044 \pm 0.003$ & $2.729 \pm 0.004$ & $2.159 \pm 0.004$ & $0.813 \pm 0.011$ & $-1.420 \pm 0.036$ \\
\hline & 0.0150 & $10.210 \pm 0.001$ & $4.804 \pm 0.005$ & $3.203 \pm 0.001$ & $2.628 \pm 0.002$ & $1.307 \pm 0.007$ & $-0.878 \pm 0.021$ \\
\hline & 0.0200 & $10.738 \pm 0.001$ & $5.127 \pm 0.006$ & $3.404 \pm 0.007$ & $2.827 \pm 0.006$ & $1.507 \pm 0.003$ & $-0.651 \pm 0.014$ \\
\hline \multirow[t]{7}{*}{13} & 0.0001 & $4.753 \pm 0.007$ & $1.795 \pm 0.004$ & $0.756 \pm 0.007$ & $0.238 \pm 0.014$ & $-0.930 \pm 0.027$ & $-2.616 \pm 0.044$ \\
\hline & 0.0008 & $5.504 \pm 0.003$ & $2.243 \pm 0.005$ & $1.203 \pm 0.007$ & $0.645 \pm 0.010$ & $-0.593 \pm 0.020$ & $-2.378 \pm 0.042$ \\
\hline & 0.0018 & $6.442 \pm 0.001$ & $2.791 \pm 0.004$ & $1.710 \pm 0.014$ & $1.136 \pm 0.018$ & $-0.163 \pm 0.024$ & $-2.110 \pm 0.037$ \\
\hline & 0.0037 & $7.283 \pm 0.000$ & $3.358 \pm 0.005$ & $2.160 \pm 0.008$ & $1.571 \pm 0.008$ & $0.212 \pm 0.010$ & $-1.878 \pm 0.008$ \\
\hline & 0.0075 & $8.441 \pm 0.000$ & $4.071 \pm 0.002$ & $2.713 \pm 0.007$ & $2.135 \pm 0.008$ & $0.778 \pm 0.012$ & $-1.443 \pm 0.018$ \\
\hline & 0.0150 & $9.819 \pm 0.000$ & $4.796 \pm 0.000$ & $3.195 \pm 0.003$ & $2.616 \pm 0.003$ & $1.277 \pm 0.008$ & $-0.922 \pm 0.022$ \\
\hline & 0.0200 & $10.379 \pm 0.001$ & $5.111 \pm 0.003$ & $3.391 \pm 0.005$ & $2.809 \pm 0.006$ & $1.473 \pm 0.012$ & $-0.697 \pm 0.017$ \\
\hline \multirow[t]{7}{*}{11} & 0.0001 & $4.975 \pm 0.003$ & $1.781 \pm 0.004$ & $0.722 \pm 0.012$ & $0.182 \pm 0.018$ & $-1.041 \pm 0.032$ & $-2.814 \pm 0.051$ \\
\hline & 0.0008 & $5.562 \pm 0.001$ & $2.256 \pm 0.005$ & $1.171 \pm 0.009$ & $0.594 \pm 0.006$ & $-0.687 \pm 0.020$ & $-2.552 \pm 0.040$ \\
\hline & 0.0018 & $6.244 \pm 0.001$ & $2.818 \pm 0.005$ & $1.685 \pm 0.005$ & $1.088 \pm 0.004$ & $2 \pm 0$ & $-2.245 \pm 0.035$ \\
\hline & 0.0037 & $6.978 \pm 0.001$ & $3.362 \pm 0.002$ & $2.138 \pm 0.010$ & $1.545 \pm 0.013$ & $0.186 \pm 0.019$ & $-1.908 \pm 0.021$ \\
\hline & 0.0075 & $8.027 \pm 0.000$ & $4.031 \pm 0.001$ & $2.668 \pm 0.006$ & $2.082 \pm 0.009$ & $0.717 \pm 0.017$ & $-1.485 \pm 0.026$ \\
\hline & 0.0150 & $9.329 \pm 0.001$ & $4.746 \pm 0.000$ & $3.179 \pm 0.006$ & $2.595 \pm 0.008$ & $1.243 \pm 0.014$ & $-0.972 \pm 0.021$ \\
\hline & 0.0200 & $9.893 \pm 0.000$ & $5.040 \pm 0.001$ & $3.382 \pm 0.002$ & $2.796 \pm 0.003$ & $1.440 \pm 0.004$ & $-0.771 \pm 0.014$ \\
\hline \multirow[t]{7}{*}{9} & 0.0001 & $4.897 \pm 0.002$ & $1.750 \pm 0.008$ & $0.664 \pm 0.018$ & $0.098 \pm 0.027$ & $-1.186 \pm 0.051$ & $-3.027 \pm 0.075$ \\
\hline & 0.0008 & $5.356 \pm 0.001$ & $2.233 \pm 0.006$ & $1.137 \pm 0.006$ & $0.545 \pm 0.014$ & $-0.765 \pm 0.020$ & $-2.679 \pm 0.028$ \\
\hline & 0.0018 & $5.916 \pm 0.001$ & $2.802 \pm 0.003$ & $1.643 \pm 0.014$ & $1.041 \pm 0.017$ & $-0.302 \pm 0.020$ & $-2.296 \pm 0.030$ \\
\hline & 0.0037 & $6.608 \pm 0.001$ & $3.345 \pm 0.004$ & $2.094 \pm 0.003$ & $1.490 \pm$ & $2 \pm 0.003$ & $-2.000 \pm 0.010$ \\
\hline & 0.0075 & $7.560 \pm 0.001$ & $3.970 \pm 0.001$ & $2.629 \pm 0.004$ & $2.040 \pm 0.006$ & $8 \pm 0.014$ & $-1.524 \pm 0.034$ \\
\hline & 0.0150 & $8.780 \pm 0.001$ & $4.644 \pm 0.001$ & $3.143 \pm 0.001$ & $2.555 \pm 0.002$ & $1.186 \pm 0.005$ & $-1.034 \pm 0.014$ \\
\hline & 0.0200 & $9.350 \pm 0.000$ & $4.921 \pm 0.004$ & $3.341 \pm 0.005$ & $2.753 \pm 0.007$ & $1.389 \pm 0.015$ & $-0.811 \pm 0.028$ \\
\hline \multirow[t]{7}{*}{7} & 0.0001 & $4.619 \pm 0.001$ & $1.713 \pm 0.006$ & $0.617 \pm 0.006$ & $0.014 \pm 0.011$ & $-1.354 \pm 0.018$ & $-3.341 \pm 0.036$ \\
\hline & 0.0008 & $4.982 \pm 0.001$ & $2.170 \pm 0.007$ & $1.046 \pm 0.007$ & $0.422 \pm 0.012$ & $-0.969 \pm 0.013$ & $-3.024 \pm 0.008$ \\
\hline & 0.0018 & $5.484 \pm 0.001$ & $2.747 \pm 0.003$ & $1.580 \pm 0.006$ & $0.969 \pm 0.008$ & $-0.406 \pm 0.020$ & $-2.471 \pm 0.056$ \\
\hline & 0.0037 & $6.159 \pm 0.001$ & $3.269 \pm 0.001$ & $2.029 \pm 0.004$ & $1.414 \pm 0.005$ & $0.014 \pm 0.007$ & $-2.109 \pm 0.017$ \\
\hline & 0.0075 & $7.020 \pm 0.001$ & $3.855 \pm 0.002$ & $2.565 \pm 0.007$ & $1.966 \pm$ & $6 \pm 0.009$ & $-1.688 \pm 0.019$ \\
\hline & 0.0150 & $8.201 \pm 0.001$ & $4.488 \pm 0.002$ & $3.083 \pm 0.004$ & $2.490 \pm 0.003$ & $1.104 \pm 0.004$ & $-1.123 \pm 0.017$ \\
\hline & 0.0200 & $8.766 \pm 0.001$ & $4.753 \pm 0.002$ & $3.304 \pm 0.005$ & $2.717 \pm 0.006$ & $1.337 \pm 0.009$ & $-0.881 \pm 0.009$ \\
\hline \multirow[t]{7}{*}{5} & 0.0001 & $4.112 \pm 0.001$ & $1.605 \pm 0.002$ & $0.499 \pm 0.009$ & $-0.147 \pm 0.016$ & $-1.612 \pm 0.031$ & $-3.698 \pm 0.038$ \\
\hline & 0.0008 & $4.429 \pm 0.001$ & $2.092 \pm 0.004$ & $1.016 \pm 0.022$ & $0.381 \pm 0.027$ & $-1.068 \pm 0.034$ & $-3.197 \pm 0.025$ \\
\hline & 0.0018 & $4.890 \pm 0.001$ & $2.641 \pm 0.005$ & $1.523 \pm 0.015$ & $0.904 \pm 0.020$ & $-0.495 \pm 0.024$ & $-2.608 \pm 0.053$ \\
\hline & 0.0037 & $5.539 \pm 0.001$ & $3.131 \pm 0.001$ & $1.964 \pm 0.003$ & $1.347 \pm 0.003$ & $-0.070 \pm 0.012$ & $-2.311 \pm 0.033$ \\
\hline & 0.0075 & $6.351 \pm 0.000$ & $3.700 \pm 0.001$ & $2.573 \pm 0.005$ & $1.983 \pm 0.004$ & $0.580 \pm 0.006$ & $-1.687 \pm 0.027$ \\
\hline & 0.0150 & $7.493 \pm 0.001$ & $4.229 \pm 0.000$ & $2.970 \pm 0.003$ & $2.384 \pm 0.003$ & $0.895 \pm 0.012$ & $-1.245 \pm 0.035$ \\
\hline & 0.0200 & $8.052 \pm 0.001$ & $4.451 \pm 0.001$ & $3.130 \pm 0.004$ & $2.549 \pm 0.004$ & $1.170 \pm 0.004$ & $-1.056 \pm 0.014$ \\
\hline \multirow[t]{7}{*}{3} & 0.0001 & $3.305 \pm 0.001$ & $1.399 \pm 0.002$ & $0.298 \pm 0.019$ & $-0.399 \pm 0.032$ & $-1.959 \pm 0.054$ & $-4.120 \pm 0.070$ \\
\hline & 0.0008 & $3.304 \pm 0.000$ & $1.400 \pm 0.000$ & $0.231 \pm 0.000$ & $-0.308 \pm 0.000$ & $-2.131 \pm 0.000$ & $-4.330 \pm 0.000$ \\
\hline & 0.0018 & $4.097 \pm 0.001$ & $2.357 \pm 0.002$ & $1.336 \pm 0.009$ & $0.697 \pm 0.010$ & $-0.821 \pm 0.013$ & $-3.152 \pm 0.023$ \\
\hline & 0.0037 & $4.665 \pm 0.001$ & $2.775 \pm 0.001$ & $1.692 \pm 0.003$ & $1.078 \pm 0.005$ & $-0.368 \pm 0.007$ & $-2.662 \pm 0.013$ \\
\hline & 0.0075 & $5.457 \pm 0.000$ & $3.289 \pm 0.000$ & $2.191 \pm 0.003$ & $1.593 \pm 0.004$ & $0.178 \pm 0.001$ & $-2.107 \pm 0.039$ \\
\hline & 0.0150 & $6.426 \pm 0.001$ & $3.760 \pm 0.001$ & $2.669 \pm 0.004$ & $2.093 \pm 0.006$ & $0.709 \pm 0.007$ & $-1.505 \pm 0.009$ \\
\hline & 0.0200 & $6.923 \pm 0.001$ & $3.963 \pm 0.000$ & $2.857 \pm 0.003$ & $2.288 \pm 0.004$ & $0.915 \pm 0.005$ & $-1.273 \pm 0.013$ \\
\hline
\end{tabular}


A. Marín-Franch and A. Aparicio: Surface-brightness fluctuations in stellar populations, Online Material p 13

Table 9. HST WFPC2 and ACS filters: SSP integrated colors obtained with the G00 stellar evolution library.

\begin{tabular}{|c|c|c|c|c|c|c|}
\hline Gy & $\overline{\bar{Z}}$ & $\overline{(F 218 W-F 336 W)}$ & $\bar{~}(F 336 W-F 439 W)$ & 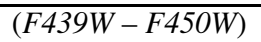 & 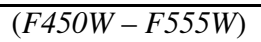 & $(F 555 W-F 814 W)$ \\
\hline \multirow[t]{7}{*}{15} & 0.0001 & $2.030 \pm 0.002$ & $-0.061 \pm 0.002$ & $0.204 \pm 0.003$ & $0.514 \pm 0.005$ & $0.913 \pm 0.007$ \\
\hline & 0.0008 & $2.295 \pm 0.002$ & $-0.024 \pm 0.003$ & $0.222 \pm 0.003$ & $0.554 \pm 0.003$ & $0.952 \pm 0.004$ \\
\hline & 0.0018 & $2.715 \pm 0.003$ & $0.067 \pm 0.005$ & $0.249 \pm 0.006$ & $0.609 \pm 0.008$ & $1.005 \pm 0.010$ \\
\hline & 0.0037 & $3.271 \pm 0.002$ & $0.194 \pm 0.003$ & $0.275 \pm 0.004$ & $0.668 \pm 0.004$ & $1.068 \pm 0.007$ \\
\hline & 0.0075 & $3.970 \pm 0.001$ & $0.346 \pm 0.002$ & $0.300 \pm 0.002$ & $0.731 \pm 0.003$ & $1.137 \pm 0.004$ \\
\hline & 0.0150 & $5.040 \pm 0.003$ & $0.560 \pm 0.003$ & $0.326 \pm 0.003$ & $0.799 \pm 0.003$ & $1.199 \pm 0.004$ \\
\hline & 0.0200 & $5.537 \pm 0.003$ & $0.655 \pm 0.004$ & $0.336 \pm 0.004$ & $0.824 \pm 0.004$ & $1.216 \pm 0.004$ \\
\hline \multirow[t]{7}{*}{13} & 0.0001 & $2.053 \pm 0.003$ & $-0.080 \pm 0.006$ & $0.206 \pm 0.008$ & $0.519 \pm 0.009$ & $0.923 \pm 0.010$ \\
\hline & 0.0008 & $2.244 \pm 0.001$ & $-0.033 \pm 0.002$ & $0.222 \pm 0.003$ & $0.552 \pm 0.003$ & $0.953 \pm 0.005$ \\
\hline & 0.0018 & $2.602 \pm 0.001$ & $0.051 \pm 0.002$ & $0.246 \pm 0.003$ & $0.603 \pm 0.004$ & $1.000 \pm 0.005$ \\
\hline & 0.0037 & $3.119 \pm 0.001$ & $0.168 \pm 0.004$ & $0.269 \pm 0.007$ & $0.658 \pm 0.010$ & $1.059 \pm 0.016$ \\
\hline & 0.0075 & $3.776 \pm 0.003$ & $0.308 \pm 0.003$ & $0.293 \pm 0.003$ & $0.715 \pm 0.003$ & $1.123 \pm 0.003$ \\
\hline & 0.0150 & $4.822 \pm 0.002$ & $0.516 \pm 0.003$ & $0.320 \pm 0.004$ & $0.785 \pm 0.005$ & $1.182 \pm 0.009$ \\
\hline & 0.0200 & $5.298 \pm 0.002$ & $0.604 \pm 0.001$ & $0.329 \pm 0.002$ & $0.809 \pm 0.002$ & $1.202 \pm 0.003$ \\
\hline \multirow[t]{7}{*}{11} & 0.0001 & $1.964 \pm 0.003$ & $-0.087 \pm 0.005$ & $0.206 \pm 0.007$ & $0.518 \pm 0.009$ & $0.926 \pm 0.010$ \\
\hline & 0.0008 & $2.135 \pm 0.001$ & $-0.043 \pm 0.003$ & $0.220 \pm 0.005$ & $0.549 \pm 0.006$ & $0.954 \pm 0.010$ \\
\hline & 0.0018 & $2.461 \pm 0.002$ & $0.032 \pm 0.004$ & $0.241 \pm 0.006$ & $0.595 \pm 0.007$ & $0.998 \pm 0.009$ \\
\hline & 0.0037 & $2.934 \pm 0.001$ & $0.137 \pm 0.002$ & $0.263 \pm 0.003$ & $0.646 \pm 0.002$ & $1.050 \pm 0.005$ \\
\hline & 0.0075 & $3.579 \pm 0.004$ & $0.279 \pm 0.004$ & $0.288 \pm 0.004$ & $0.705 \pm 0.004$ & $1.112 \pm 0.005$ \\
\hline & 0.0150 & $4.572 \pm 0.001$ & $0.465 \pm 0.001$ & $0.311 \pm 0.001$ & $0.767 \pm 0.001$ & $1.163 \pm 0.004$ \\
\hline & 0.0200 & $5.011 \pm 0.001$ & $0.544 \pm 0.001$ & $0.320 \pm 0.000$ & $0.791 \pm 0.001$ & $1.183 \pm 0.003$ \\
\hline \multirow[t]{7}{*}{9} & 0.0001 & $1.853 \pm 0.002$ & $-0.088 \pm 0.004$ & $0.202 \pm 0.006$ & $0.511 \pm 0.009$ & $0.923 \pm 0.013$ \\
\hline & 0.0008 & $2.010 \pm 0.003$ & $-0.054 \pm 0.006$ & $0.214 \pm 0.009$ & $0.538 \pm 0.011$ & $0.946 \pm 0.016$ \\
\hline & 0.0018 & $2.305 \pm 0.002$ & $0.011 \pm 0.004$ & $0.233 \pm 0.006$ & $0.580 \pm 0.007$ & $0.982 \pm 0.010$ \\
\hline & 0.0037 & $2.745 \pm 0.002$ & $0.109 \pm 0.003$ & $0.255 \pm 0.004$ & $0.630 \pm 0.006$ & $1.032 \pm 0.009$ \\
\hline & 0.0075 & $3.364 \pm 0.001$ & $0.236 \pm 0.001$ & $0.278 \pm 0.001$ & $0.686 \pm 0.004$ & $1.094 \pm 0.011$ \\
\hline & 0.0150 & $4.294 \pm 0.001$ & $0.407 \pm 0.003$ & $0.301 \pm 0.004$ & $0.746 \pm 0.005$ & $1.141 \pm 0.008$ \\
\hline & 0.0200 & $4.692 \pm 0.001$ & $0.481 \pm 0.001$ & $0.310 \pm 0.002$ & $0.769 \pm 0.003$ & $1.159 \pm 0.005$ \\
\hline \multirow[t]{7}{*}{7} & 0.0001 & $1.688 \pm 0.002$ & $-0.088 \pm 0.001$ & $0.194 \pm 0.002$ & $0.498 \pm 0.003$ & $0.915 \pm 0.006$ \\
\hline & 0.0008 & $1.836 \pm 0.003$ & $-0.059 \pm 0.003$ & $0.207 \pm 0.003$ & $0.527 \pm 0.002$ & $0.941 \pm 0.007$ \\
\hline & 0.0018 & $2.115 \pm 0.001$ & $-0.007 \pm 0.001$ & $0.224 \pm 0.001$ & $0.563 \pm 0.002$ & $0.968 \pm 0.003$ \\
\hline & 0.0037 & $2.525 \pm 0.001$ & $0.078 \pm 0.002$ & $0.245 \pm 0.004$ & $0.609 \pm 0.006$ & $1.013 \pm 0.015$ \\
\hline & 0.0075 & $3.117 \pm 0.002$ & $0.190 \pm 0.001$ & $0.267 \pm 0.002$ & $0.663 \pm 0.002$ & $1.066 \pm 0.003$ \\
\hline & 0.0150 & $3.965 \pm 0.001$ & $0.344 \pm 0.001$ & $0.289 \pm 0.002$ & $0.720 \pm 0.002$ & $1.114 \pm 0.002$ \\
\hline & 0.0200 & $4.346 \pm 0.002$ & $0.410 \pm 0.002$ & $0.296 \pm 0.003$ & $0.741 \pm 0.004$ & $1.126 \pm 0.006$ \\
\hline \multirow[t]{7}{*}{5} & 0.0001 & $1.439 \pm 0.002$ & $-0.066 \pm 0.003$ & $0.182 \pm 0.005$ & $0.478 \pm 0.006$ & $0.899 \pm 0.007$ \\
\hline & 0.0008 & $1.590 \pm 0.002$ & $-0.053 \pm 0.002$ & $0.195 \pm 0.002$ & $0.505 \pm 0.001$ & $0.922 \pm 0.000$ \\
\hline & 0.0018 & $1.846 \pm 0.002$ & $-0.016 \pm 0.002$ & $0.212 \pm 0.002$ & $0.543 \pm 0.002$ & $0.955 \pm 0.003$ \\
\hline & 0.0037 & $2.245 \pm 0.001$ & $0.049 \pm 0.002$ & $0.232 \pm 0.002$ & $0.585 \pm 0.004$ & $0.991 \pm 0.009$ \\
\hline & 0.0075 & $2.779 \pm 0.001$ & $0.145 \pm 0.001$ & $0.254 \pm 0.001$ & $0.638 \pm 0.001$ & $1.045 \pm 0.004$ \\
\hline & 0.0150 & $3.626 \pm 0.001$ & $0.301 \pm 0.002$ & $0.280 \pm 0.002$ & $0.701 \pm 0.003$ & $1.096 \pm 0.004$ \\
\hline & 0.0200 & $4.015 \pm 0.001$ & $0.365 \pm 0.002$ & $0.289 \pm 0.003$ & $0.723 \pm 0.004$ & $1.112 \pm 0.005$ \\
\hline \multirow[t]{7}{*}{3} & 0.0001 & $1.091 \pm 0.002$ & $-0.019 \pm 0.003$ & $0.160 \pm 0.003$ & $0.440 \pm 0.005$ & $0.867 \pm 0.010$ \\
\hline & 0.0008 & $1.234 \pm 0.002$ & $-0.014 \pm 0.002$ & $0.172 \pm 0.002$ & $0.465 \pm 0.003$ & $0.890 \pm 0.007$ \\
\hline & 0.0018 & $1.492 \pm 0.002$ & $-0.005 \pm 0.002$ & $0.189 \pm 0.002$ & $0.499 \pm 0.003$ & $0.915 \pm 0.011$ \\
\hline & 0.0037 & $1.897 \pm 0.002$ & $0.037 \pm 0.003$ & $0.214 \pm 0.004$ & $0.552 \pm 0.004$ & $0.966 \pm 0.006$ \\
\hline & 0.0075 & $2.367 \pm 0.002$ & $0.095 \pm 0.001$ & $0.233 \pm 0.001$ & $0.597 \pm 0.003$ & $1.010 \pm 0.009$ \\
\hline & 0.0150 & $3.097 \pm 0.002$ & $0.208 \pm 0.002$ & $0.256 \pm 0.001$ & $0.651 \pm 0.002$ & $1.043 \pm 0.005$ \\
\hline & 0.0200 & $3.460 \pm 0.002$ & $0.262 \pm 0.001$ & $0.264 \pm 0.001$ & $0.673 \pm 0.002$ & $1.057 \pm 0.003$ \\
\hline
\end{tabular}


A. Marín-Franch and A. Aparicio: Surface-brightness fluctuations in stellar populations, Online Material p 14

Table 10. HST WFPC2 and ACS filters: SSP fluctuation magnitudes obtained with the G00 stellar evolution library.

\begin{tabular}{|c|c|c|c|c|c|c|c|}
\hline Gy & $Z$ & $\bar{M}_{F 218 W}$ & $\bar{M}_{F 336 W}$ & $\bar{M}_{F 439 W}$ & $\bar{M}_{F 450 W}$ & $\bar{M}_{F 555 W}$ & $\bar{M}_{F 814 W}$ \\
\hline \multirow[t]{7}{*}{15} & 0.0001 & $4.028 \pm 0.003$ & $1.912 \pm 0.002$ & $0.932 \pm 0.014$ & $0.434 \pm 0.019$ & $-0.711 \pm 0.024$ & $-2.391 \pm 0.033$ \\
\hline & 0.0008 & $5.183 \pm 0.008$ & $2.247 \pm 0.003$ & $1.244 \pm 0.006$ & $0.694 \pm 0.011$ & $-0.540 \pm 0.020$ & $-2.323 \pm 0.024$ \\
\hline & 0.0018 & $6.422 \pm 0.001$ & $2.718 \pm 0.003$ & $1.654 \pm 0.008$ & $1.070 \pm 0.012$ & $-0.248 \pm 0.026$ & $-2.184 \pm 0.039$ \\
\hline & 0.0037 & $7.512 \pm 0.000$ & $3.255 \pm 0.005$ & $2.107 \pm 0.005$ & $1.510 \pm 0.010$ & $0.136 \pm 0.023$ & $-1.935 \pm 0.045$ \\
\hline & 0.0075 & $8.608 \pm 0.001$ & $3.956 \pm 0.003$ & $2.608 \pm 0.002$ & $2.004 \pm 0.002$ & $0.597 \pm 0.012$ & $-1.616 \pm 0.035$ \\
\hline & 0.0150 & $10.151 \pm 0.000$ & $4.805 \pm 0.008$ & $3.171 \pm 0.005$ & $2.570 \pm 0.006$ & $1.176 \pm 0.006$ & $-1.060 \pm 0.010$ \\
\hline & 0.0200 & $10.863 \pm 0.001$ & $5.165 \pm 0.002$ & $3.395 \pm 0.004$ & $2.799 \pm 0.006$ & $1.418 \pm 0.009$ & $-0.813 \pm 0.010$ \\
\hline \multirow[t]{7}{*}{13} & 0.0001 & $4.916 \pm 0.001$ & $1.925 \pm 0.012$ & $0.909 \pm 0.012$ & $0.380 \pm 0.012$ & $-0.829 \pm 0.020$ & $-2.602 \pm 0.038$ \\
\hline & 0.0008 & $5.551 \pm 0.001$ & $2.249 \pm 0.003$ & $1.201 \pm 0.007$ & $0.638 \pm 0.008$ & $-0.626 \pm 0.007$ & $-2.470 \pm 0.018$ \\
\hline & 0.0018 & $6.408 \pm 0.002$ & $2.744 \pm 0.002$ & $1.635 \pm 0.006$ & $1.039 \pm 0.004$ & $-0.297 \pm 0.006$ & $-2.258 \pm 0.021$ \\
\hline & 0.0037 & $7.294 \pm 0.000$ & $3.282 \pm 0.001$ & $2.099 \pm 0.018$ & $1.494 \pm 0.022$ & $0.107 \pm 0.027$ & $-1.991 \pm 0.025$ \\
\hline & 0.0075 & $8.299 \pm 0.000$ & $3.983 \pm 0.005$ & $2.613 \pm 0.009$ & $2.001 \pm 0.010$ & $0.576 \pm 0.004$ & $-1.633 \pm 0.024$ \\
\hline & 0.0150 & $9.782 \pm 0.001$ & $4.791 \pm 0.007$ & $3.163 \pm 0.008$ & $2.556 \pm 0.008$ & $1.148 \pm 0.008$ & $-1.101 \pm 0.032$ \\
\hline & 0.0200 & $10.458 \pm 0.001$ & $5.120 \pm 0.003$ & $3.378 \pm 0.003$ & $2.777 \pm 0.004$ & $1.379 \pm 0.003$ & $-0.864 \pm 0.008$ \\
\hline \multirow[t]{7}{*}{11} & 0.0001 & $5.034 \pm 0.002$ & $1.913 \pm 0.001$ & $0.840 \pm 0.008$ & $0.281 \pm 0.010$ & $-0.987 \pm 0.017$ & $-2.835 \pm 0.027$ \\
\hline & 0.0008 & $5.516 \pm 0.001$ & $2.238 \pm 0.003$ & $1.151 \pm 0.006$ & $0.564 \pm 0.012$ & $-0.751 \pm 0.030$ & $-2.659 \pm 0.035$ \\
\hline & 0.0018 & $6.218 \pm 0.001$ & $2.751 \pm 0.004$ & $1.604 \pm 0.010$ & $0.994 \pm 0.009$ & $-0.369 \pm 0.017$ & $-2.374 \pm 0.062$ \\
\hline & 0.0037 & $6.989 \pm 0.000$ & $3.313 \pm 0.004$ & $2.088 \pm 0.004$ & $1.472 \pm 0.004$ & $0.064 \pm 0.016$ & $-2.077 \pm 0.033$ \\
\hline & 0.0075 & $7.944 \pm 0.000$ & $3.971 \pm 0.006$ & $2.577 \pm 0.006$ & $1.957 \pm 0.007$ & $0.520 \pm 0.007$ & $-1.722 \pm 0.027$ \\
\hline & 0.0150 & $9.352 \pm 0.000$ & $4.737 \pm 0.002$ & $3.142 \pm 0.004$ & $2.532 \pm 0.004$ & $1.112 \pm 0.011$ & $-1.138 \pm 0.039$ \\
\hline & 0.0200 & $9.970 \pm 0.001$ & $5.051 \pm 0.003$ & $3.350 \pm 0.004$ & $2.741 \pm 0.007$ & $1.323 \pm 0.016$ & $-0.931 \pm 0.023$ \\
\hline \multirow[t]{7}{*}{9} & 0.0001 & $4.975 \pm 0.002$ & $1.869 \pm 0.005$ & $0.784 \pm 0.021$ & $0.205 \pm 0.023$ & $-1.114 \pm 0.034$ & $-3.033 \pm 0.051$ \\
\hline & 0.0008 & $5.345 \pm 0.001$ & $2.201 \pm 0.006$ & $1.113 \pm 0.023$ & $0.515 \pm 0.026$ & $-0.834 \pm 0.027$ & $-2.820 \pm 0.031$ \\
\hline & 0.0018 & $5.923 \pm 0.000$ & $2.727 \pm 0.005$ & $1.584 \pm 0.011$ & $0.970 \pm 0.014$ & $-0.415 \pm 0.014$ & $-2.500 \pm 0.015$ \\
\hline & 0.0037 & $6.613 \pm 0.001$ & $3.274 \pm 0.002$ & $2.052 \pm 0.006$ & $1.433 \pm 0.007$ & $0.017 \pm 0.012$ & $-2.170 \pm 0.031$ \\
\hline & 0.0075 & $7.553 \pm 0.001$ & $3.931 \pm 0.000$ & $2.555 \pm 0.007$ & $1.930 \pm 0.012$ & $0.474 \pm 0.028$ & $-1.777 \pm 0.052$ \\
\hline & 0.0150 & $8.870 \pm 0.001$ & $4.643 \pm 0.002$ & $3.108 \pm 0.005$ & $2.494 \pm 0.006$ & $1.064 \pm 0.009$ & $-1.198 \pm 0.021$ \\
\hline & 0.0200 & $9.416 \pm 0.001$ & $4.923 \pm 0.002$ & $3.320 \pm 0.004$ & $2.713 \pm 0.006$ & $1.295 \pm 0.011$ & $-0.971 \pm 0.023$ \\
\hline \multirow[t]{7}{*}{7} & 0.0001 & $4.677 \pm 0.001$ & $1.814 \pm 0.005$ & $0.736 \pm 0.010$ & $0.128 \pm 0.011$ & $-1.264 \pm 0.007$ & $-3.286 \pm 0.009$ \\
\hline & 0.0008 & $4.999 \pm 0.001$ & $2.144 \pm 0.004$ & $1.046 \pm 0.003$ & $0.421 \pm 0.011$ & $-0.999 \pm 0.028$ & $-3.095 \pm 0.055$ \\
\hline & 0.0018 & $5.507 \pm 0.001$ & $2.655 \pm 0.002$ & $1.535 \pm 0.006$ & $0.917 \pm 0.005$ & $-0.496 \pm 0.011$ & $-2.631 \pm 0.030$ \\
\hline & 0.0037 & $6.151 \pm 0.001$ & $3.188 \pm 0.001$ & $1.993 \pm 0.007$ & $1.374 \pm 0.012$ & $-0.042 \pm 0.033$ & $3 \pm 0.066$ \\
\hline & 0.0075 & $7.068 \pm 0.001$ & $3.824 \pm 0.003$ & $2.502 \pm 0.002$ & $1.878 \pm 0.004$ & $0.430 \pm 0.015$ & $-1.815 \pm 0.031$ \\
\hline & 0.0150 & $8.258 \pm 0.000$ & $4.471 \pm 0.002$ & $3.037 \pm 0.007$ & $2.423 \pm 0.007$ & $0.980 \pm 0.008$ & $-1.285 \pm 0.011$ \\
\hline & 0.0200 & $8.798 \pm 0.000$ & $4.751 \pm 0.001$ & $3.284 \pm 0.006$ & $2.679 \pm 0.007$ & $1.253 \pm 0.010$ & $-1.011 \pm 0.022$ \\
\hline \multirow[t]{7}{*}{5} & 0.0001 & $4.158 \pm 0.001$ & $1.705 \pm 0.003$ & $0.612 \pm 0.004$ & $-0.042 \pm 0.014$ & $-1.524 \pm 0.030$ & $-3.633 \pm 0.040$ \\
\hline & 0.0008 & $4.462 \pm 0.001$ & $2.045 \pm 0.001$ & $0.962 \pm 0.006$ & $0.309 \pm 0.003$ & $-1.186 \pm 0.014$ & $-3.372 \pm 0.039$ \\
\hline & 0.0018 & $4.911 \pm 0.000$ & $2.527 \pm 0.001$ & $1.426 \pm 0.006$ & $0.788 \pm 0.004$ & $-0.692 \pm 0.013$ & $-2.929 \pm 0.027$ \\
\hline & 0.0037 & $5.536 \pm 0.001$ & $3.029 \pm 0.002$ & $1.881 \pm 0.005$ & $1.254 \pm 0.009$ & $-0.197 \pm 0.018$ & $-2.451 \pm 0.024$ \\
\hline & 0.0075 & $6.344 \pm 0.000$ & $3.620 \pm 0.001$ & $2.388 \pm 0.003$ & $1.761 \pm 0.006$ & $0.294 \pm 0.014$ & $-1.988 \pm 0.018$ \\
\hline & 0.0150 & $7.580 \pm 0.000$ & $4.213 \pm 0.001$ & $2.894 \pm 0.005$ & $2.283 \pm 0.007$ & $0.840 \pm 0.006$ & $-1.425 \pm 0.014$ \\
\hline & 0.0200 & $8.145 \pm 0.000$ & $4.457 \pm 0.001$ & $3.108 \pm 0.008$ & $2.505 \pm 0.009$ & $1.064 \pm 0.011$ & $-1.192 \pm 0.016$ \\
\hline \multirow[t]{7}{*}{3} & 0.0001 & $3.328 \pm 0.001$ & $1.494 \pm 0.004$ & $0.448 \pm 0.011$ & $-0.248 \pm 0.017$ & $-1.829 \pm 0.029$ & $-4.043 \pm 0.041$ \\
\hline & 0.0008 & $3.613 \pm 0.001$ & $1.839 \pm 0.002$ & $0.827 \pm 0.005$ & $0.141 \pm 0.010$ & $-1.454 \pm 0.025$ & $-3.754 \pm 0.029$ \\
\hline & 0.0018 & $4.078 \pm 0.001$ & $2.314 \pm 0.002$ & $1.307 \pm 0.006$ & $0.656 \pm 0.016$ & $-0.889 \pm 0.038$ & $-3.239 \pm 0.064$ \\
\hline & 0.0037 & $4.725 \pm 0.001$ & $2.760 \pm 0.001$ & $1.709 \pm 0.002$ & $1.082 \pm 0.002$ & $-0.420 \pm 0.008$ & $-2.822 \pm 0.019$ \\
\hline & 0.0075 & $5.516 \pm 0.001$ & $3.305 \pm 0.001$ & $2.220 \pm 0.008$ & $1.598 \pm 0.012$ & $0.103 \pm 0.023$ & $-2.289 \pm 0.037$ \\
\hline & 0.0150 & $6.611 \pm 0.000$ & $3.800 \pm 0.000$ & $2.707 \pm 0.005$ & $2.107 \pm 0.007$ & $0.655 \pm 0.009$ & $-1.669 \pm 0.014$ \\
\hline & 0.0200 & $7.112 \pm 0.042$ & $4.003 \pm 0.001$ & $2.894 \pm 0.001$ & $2.303 \pm 0.002$ & $0.864 \pm 0.003$ & $-1.400 \pm 0.012$ \\
\hline
\end{tabular}


A. Marín-Franch and A. Aparicio: Surface-brightness fluctuations in stellar populations, Online Material p 15

Table 11. HST WFPC2 and ACS filters: SSP integrated colors obtained with the P04 stellar evolution library.

\begin{tabular}{|c|c|c|c|c|c|c|}
\hline Gy & $\bar{Z}$ & $(F 218 W-F 336 W)$ & $(F 336 W-F 439 W)$ & $(F 439 W-F 450 W)$ & $(F 450 W-F 555 W)$ & $(F 555 W-F 814 W)$ \\
\hline \multirow[t]{7}{*}{13} & 0.0004 & $1.301 \pm 0.006$ & $-0.050 \pm 0.006$ & $0.198 \pm 0.008$ & $0.510 \pm 0.010$ & $0.940 \pm 0.012$ \\
\hline & 0.0008 & $1.566 \pm 0.004$ & $0.033 \pm 0.003$ & $0.205 \pm 0.005$ & $0.527 \pm 0.008$ & $0.952 \pm 0.012$ \\
\hline & 0.0018 & $1.913 \pm 0.004$ & $0.097 \pm 0.003$ & $0.220 \pm 0.003$ & $0.567 \pm 0.002$ & $0.987 \pm 0.002$ \\
\hline & 0.0037 & $3.017 \pm 0.002$ & $0.155 \pm 0.003$ & $0.264 \pm 0.003$ & $0.653 \pm 0.004$ & $1.071 \pm 0.008$ \\
\hline & 0.0075 & $3.824 \pm 0.002$ & $0.311 \pm 0.002$ & $0.289 \pm 0.001$ & $0.705 \pm 0.002$ & $1.114 \pm 0.004$ \\
\hline & 0.0150 & $4.679 \pm 0.002$ & $0.484 \pm 0.002$ & $0.317 \pm 0.003$ & $0.773 \pm 0.005$ & $1.172 \pm 0.010$ \\
\hline & 0.0200 & $5.108 \pm 0.001$ & $0.559 \pm 0.002$ & $0.326 \pm 0.002$ & $0.793 \pm 0.004$ & $1.184 \pm 0.007$ \\
\hline \multirow[t]{7}{*}{11} & 0.0004 & $1.712 \pm 0.002$ & $-0.027 \pm 0.003$ & $0.197 \pm 0.005$ & $0.505 \pm 0.008$ & $0.925 \pm 0.013$ \\
\hline & 0.0008 & $1.985 \pm 0.002$ & $-0.008 \pm 0.003$ & $0.217 \pm 0.004$ & $0.548 \pm 0.006$ & $0.967 \pm 0.009$ \\
\hline & 0.0018 & $2.400 \pm 0.002$ & $0.042 \pm 0.002$ & $0.242 \pm 0.002$ & $0.602 \pm 0.003$ & $1.015 \pm 0.005$ \\
\hline & 0.0037 & $2.907 \pm 0.002$ & $0.142 \pm 0.003$ & $0.265 \pm 0.004$ & $0.652 \pm 0.006$ & $1.068 \pm 0.009$ \\
\hline & 0.0075 & $3.537 \pm 0.002$ & $0.276 \pm 0.001$ & $0.287 \pm 0.001$ & $0.704 \pm 0.002$ & $1.117 \pm 0.006$ \\
\hline & 0.0150 & $4.441 \pm 0.003$ & $0.452 \pm 0.005$ & $0.313 \pm 0.006$ & $0.765 \pm 0.007$ & $1.167 \pm 0.009$ \\
\hline & 0.0200 & $4.828 \pm 0.001$ & $0.512 \pm 0.004$ & $0.319 \pm 0.006$ & $0.779 \pm 0.008$ & $1.166 \pm 0.011$ \\
\hline \multirow[t]{7}{*}{9} & 0.0004 & $1.737 \pm 0.002$ & $-0.062 \pm 0.003$ & $0.201 \pm 0.004$ & $0.514 \pm 0.008$ & $0.938 \pm 0.015$ \\
\hline & 0.0008 & $1.976 \pm 0.003$ & $-0.037 \pm 0.004$ & $0.219 \pm 0.004$ & $0.552 \pm 0.004$ & $0.972 \pm 0.005$ \\
\hline & 0.0018 & $2.284 \pm 0.002$ & $0.030 \pm 0.004$ & $0.241 \pm 0.005$ & $0.597 \pm 0.006$ & $1.015 \pm 0.007$ \\
\hline & 0.0037 & $2.719 \pm 0.003$ & $0.120 \pm 0.005$ & $0.260 \pm 0.006$ & $0.643 \pm 0.006$ & $1.061 \pm 0.011$ \\
\hline & 0.0075 & $3.343 \pm 0.001$ & $0.268 \pm 0.001$ & $0.288 \pm 0.002$ & $0.705 \pm 0.002$ & $1.122 \pm 0.001$ \\
\hline & 0.0150 & $4.144 \pm 0.002$ & $0.392 \pm 0.004$ & $0.302 \pm 0.005$ & $0.745 \pm 0.006$ & $1.149 \pm 0.006$ \\
\hline & 0.0200 & $4.566 \pm 0.001$ & $0.501 \pm 0.001$ & $0.321 \pm 0.002$ & $0.785 \pm 0.003$ & $1.188 \pm 0.004$ \\
\hline \multirow[t]{7}{*}{7} & 0.0004 & $1.659 \pm 0.001$ & $-0.063 \pm 0.002$ & $0.202 \pm 0.002$ & $0.517 \pm 0.002$ & $0.944 \pm 0.005$ \\
\hline & 0.0008 & $1.823 \pm 0.001$ & $-0.034 \pm 0.002$ & $0.217 \pm 0.003$ & $0.549 \pm 0.006$ & $0.973 \pm 0.013$ \\
\hline & 0.0018 & $2.089 \pm 0.001$ & $0.015 \pm 0.004$ & $0.233 \pm 0.007$ & $0.586 \pm 0.010$ & $1.002 \pm 0.015$ \\
\hline & 0.0037 & $2.588 \pm 0.002$ & $0.150 \pm 0.003$ & $0.266 \pm 0.003$ & $0.656 \pm 0.004$ & $1.079 \pm 0.006$ \\
\hline & 0.0075 & $3.047 \pm 0.001$ & $0.208 \pm 0.002$ & $0.273 \pm 0.002$ & $0.678 \pm 0.002$ & $1.096 \pm 0.006$ \\
\hline & 0.0150 & $3.857 \pm 0.001$ & $0.369 \pm 0.002$ & $0.300 \pm 0.001$ & $0.738 \pm 0.002$ & $1.142 \pm 0.008$ \\
\hline & 0.0200 & $4.208 \pm 0.000$ & $0.421 \pm 0.001$ & $0.305 \pm 0.001$ & $0.751 \pm 0.002$ & $1.146 \pm 0.002$ \\
\hline \multirow[t]{7}{*}{5} & 0.0004 & $1.477 \pm 0.002$ & $0.006 \pm 0.003$ & $0.203 \pm 0.003$ & $0.526 \pm 0.005$ & $0.971 \pm 0.009$ \\
\hline & 0.0008 & $1.612 \pm 0.002$ & $0.025 \pm 0.001$ & $0.215 \pm 0.002$ & $0.550 \pm 0.003$ & $0.988 \pm 0.009$ \\
\hline & 0.0018 & $1.860 \pm 0.002$ & $0.052 \pm 0.003$ & $0.231 \pm 0.004$ & $0.584 \pm 0.007$ & $1.008 \pm 0.010$ \\
\hline & 0.0037 & $2.180 \pm 0.001$ & $0.092 \pm 0.003$ & $0.243 \pm 0.004$ & $0.614 \pm 0.006$ & $1.041 \pm 0.009$ \\
\hline & 0.0075 & $2.647 \pm 0.001$ & $0.149 \pm 0.001$ & $0.254 \pm 0.002$ & $0.641 \pm 0.003$ & $1.065 \pm 0.004$ \\
\hline & 0.0150 & $3.393 \pm 0.001$ & $0.246 \pm 0.001$ & $0.269 \pm 0.001$ & $0.676 \pm 0.001$ & $1.070 \pm 0.001$ \\
\hline & 0.0200 & $3.736 \pm 0.002$ & $0.296 \pm 0.002$ & $0.276 \pm 0.003$ & $0.692 \pm 0.003$ & $1.079 \pm 0.003$ \\
\hline \multirow[t]{7}{*}{3} & 0.0004 & $0.800 \pm 0.002$ & $0.019 \pm 0.002$ & $0.149 \pm 0.003$ & $0.428 \pm 0.005$ & $0.880 \pm 0.011$ \\
\hline & 0.0008 & $0.907 \pm 0.000$ & $0.035 \pm 0.001$ & $0.156 \pm 0.002$ & $0.439 \pm 0.003$ & $0.875 \pm 0.003$ \\
\hline & 0.0018 & $1.110 \pm 0.002$ & $0.039 \pm 0.004$ & $0.168 \pm 0.006$ & $0.466 \pm 0.006$ & $0.889 \pm 0.011$ \\
\hline & 0.0037 & $1.417 \pm 0.001$ & $0.032 \pm 0.003$ & $0.183 \pm 0.004$ & $0.497 \pm 0.005$ & $0.924 \pm 0.007$ \\
\hline & 0.0075 & $1.841 \pm 0.001$ & $0.047 \pm 0.001$ & $0.199 \pm 0.001$ & $0.534 \pm 0.002$ & $0.956 \pm 0.005$ \\
\hline & 0.0150 & $2.541 \pm 0.002$ & $0.103 \pm 0.001$ & $0.217 \pm 0.002$ & $0.572 \pm 0.002$ & $0.964 \pm 0.002$ \\
\hline & 0.0200 & $2.888 \pm 0.002$ & $0.140 \pm 0.001$ & $0.226 \pm 0.002$ & $0.590 \pm 0.002$ & $0.971 \pm 0.002$ \\
\hline
\end{tabular}


A. Marín-Franch and A. Aparicio: Surface-brightness fluctuations in stellar populations, Online Material p 16

Table 12. HST WFPC2 and ACS filters: SSP fluctuation magnitudes obtained with the P04 stellar evolution library.

\begin{tabular}{|c|c|c|c|c|c|c|c|}
\hline Gy & $Z$ & $\bar{M}_{F 218 W}$ & $\bar{M}_{F 336 W}$ & $\bar{M}_{F 439 W}$ & $\bar{M}_{F 450 W}$ & $\bar{M}_{F 555 W}$ & $\bar{M}_{F 814 W}$ \\
\hline \multirow[t]{7}{*}{13} & 0.0004 & $1.243 \pm 0.004$ & $1.943 \pm 0.002$ & $0.984 \pm 0.010$ & $0.478 \pm 0.010$ & $-0.736 \pm 0.010$ & $-2.486 \pm 0.005$ \\
\hline & 0.0008 & $1.693 \pm 0.003$ & $2.096 \pm 0.003$ & $1.118 \pm 0.012$ & $0.650 \pm 0.017$ & $-0.554 \pm 0.022$ & $-2.368 \pm 0.032$ \\
\hline & 0.0018 & $2.342 \pm 0.004$ & $2.411 \pm 0.004$ & $1.431 \pm 0.003$ & $0.974 \pm 0.004$ & $-0.274 \pm 0.012$ & $-2.228 \pm 0.029$ \\
\hline & 0.0037 & $6.385 \pm 0.007$ & $2.966 \pm 0.005$ & $1.988 \pm 0.006$ & $1.427 \pm 0.008$ & $0.072 \pm 0.011$ & $-2.024 \pm 0.015$ \\
\hline & 0.0075 & $8.082 \pm 0.001$ & $3.392 \pm 0.002$ & $2.363 \pm 0.004$ & $1.832 \pm 0.003$ & $0.525 \pm 0.003$ & $-1.680 \pm 0.017$ \\
\hline & 0.0150 & $9.520 \pm 0.000$ & $4.559 \pm 0.002$ & $2.968 \pm 0.011$ & $2.361 \pm 0.013$ & $0.959 \pm 0.023$ & $-1.274 \pm 0.034$ \\
\hline & 0.0200 & $10.122 \pm 0.000$ & $4.866 \pm 0.001$ & $3.166 \pm 0.008$ & $2.559 \pm 0.008$ & $1.161 \pm 0.012$ & $-1.071 \pm 0.009$ \\
\hline \multirow[t]{7}{*}{11} & 0.0004 & $2.918 \pm 0.003$ & $1.937 \pm 0.002$ & $0.936 \pm 0.014$ & $0.449 \pm 0.019$ & $-0.745 \pm 0.015$ & $-2.546 \pm 0.016$ \\
\hline & 0.0008 & $3.785 \pm 0.005$ & $2.145 \pm 0.003$ & $1.171 \pm 0.006$ & $0.638 \pm 0.009$ & $-0.621 \pm 0.010$ & $-2.481 \pm 0.014$ \\
\hline & 0.0018 & $5.730 \pm 0.004$ & $2.624 \pm 0.005$ & $1.581 \pm 0.005$ & $1.000 \pm 0.009$ & $-0.333 \pm 0.018$ & $-2.304 \pm 0.034$ \\
\hline & 0.0037 & $6.924 \pm 0.000$ & $3.204 \pm 0.003$ & $2.021 \pm 0.006$ & $1.424 \pm 0.007$ & $0.040 \pm 0.008$ & $-2.097 \pm 0.021$ \\
\hline & 0.0075 & $7.854 \pm 0.000$ & $3.844 \pm 0.002$ & $2.474 \pm 0.005$ & $1.868 \pm 0.008$ & $0.468 \pm 0$ & $-1.738 \pm 0.018$ \\
\hline & 0.0150 & $9.080 \pm 0.000$ & $4.499 \pm 0.002$ & $2.896 \pm 0.009$ & $2.285 \pm 0.008$ & $0.878 \pm 0.004$ & $-1.352 \pm 0.010$ \\
\hline & 0.0200 & $9.631 \pm 0.001$ & $4.806 \pm 0.004$ & $3.120 \pm 0.014$ & $2.508 \pm 0.015$ & $1.099 \pm 0.013$ & $-1.107 \pm 0.004$ \\
\hline \multirow[t]{7}{*}{9} & 0.0004 & $3.992 \pm 0.007$ & $1.950 \pm 0.002$ & $0.958 \pm 0.015$ & $0.411 \pm 0.021$ & $-0.873 \pm 0.030$ & $-2.775 \pm 0.031$ \\
\hline & 0.0008 & $5.068 \pm 0.004$ & $2.234 \pm 0.004$ & $1 \pm 0.006$ & $0.633 \pm 0$. & $-0.687 \pm 0$ & $-2.636 \pm 0.011$ \\
\hline & 0.0018 & $5.906 \pm 0.001$ & $2.724 \pm 0.005$ & $1.574 \pm 0.002$ & $0.967 \pm 0.002$ & $-0.405 \pm 0.005$ & $-2.437 \pm 0.011$ \\
\hline & 0.0037 & $6.583 \pm 0.001$ & $3.238 \pm 0.006$ & $1.982 \pm 0.009$ & $1.364 \pm 0.014$ & $-0.051 \pm 0.026$ & $-2.215 \pm 0.044$ \\
\hline & 0.0075 & $7.422 \pm 0.001$ & $3.728 \pm 0.003$ & $3 \pm 0.003$ & $1.723 \pm 0.002$ & $0.317 \pm 0.000$ & $-1.902 \pm 0.000$ \\
\hline & 150 & $8.581 \pm 0.000$ & $4.439 \pm 0.005$ & $3 \pm 0.006$ & $2.256 \pm 0.005$ & $3 \pm 0$ & \pm 0.014 \\
\hline & 0.0200 & $9.141 \pm 0.001$ & $4.623 \pm 0.002$ & $2.907 \pm 0.003$ & $2.286 \pm 0.004$ & $0.857 \pm 0.008$ & $-1.372 \pm 0.013$ \\
\hline \multirow[t]{7}{*}{7} & 0.0004 & $4.610 \pm 0.002$ & $1.919 \pm 0.002$ & $0.866 \pm 0.006$ & $0.280 \pm 0.010$ & $-1.044 \pm 0.019$ & $-2.981 \pm 0.030$ \\
\hline & 0.0008 & $5.000 \pm 0.000$ & $2.234 \pm 0.002$ & $2 \pm 0.013$ & $0.495 \pm 0.019$ & $4 \pm 0.027$ & \pm 0.042 \\
\hline & 0.0018 & $5.512 \pm 0.000$ & $2.699 \pm 0.004$ & \pm 0 . & $0.868 \pm 0$ & -0.5 & \pm 0.013 \\
\hline & 0.0037 & $6.120 \pm 0.001$ & $3.029 \pm 0.002$ & $8 \pm 0.003$ & $1.128 \pm 0.005$ & $-0.275 \pm 0.011$ & $-2.407 \pm 0.013$ \\
\hline & 0.0075 & $6.875 \pm 0.001$ & $3.673 \pm 0.005$ & $2.288 \pm 0.007$ & $1.657 \pm 0.008$ & $0.209 \pm 0.013$ & $-2.016 \pm 0.025$ \\
\hline & 0.0150 & $7.951 \pm 0.000$ & $4.193 \pm 0.002$ & $2.670 \pm 0.007$ & $2.044 \pm 0.009$ & $0.586 \pm 0.017$ & $-1.684 \pm 0.017$ \\
\hline & 0.0200 & $8.428 \pm 0.001$ & $4.431 \pm 0.001$ & $2.874 \pm 0.001$ & $2.255 \pm 0.001$ & $0.806 \pm 0.003$ & $-1.469 \pm 0.011$ \\
\hline \multirow[t]{7}{*}{5} & 0.0004 & $3.982 \pm 0.001$ & $1.598 \pm 0.003$ & $0.557 \pm 0.009$ & $-0.076 \pm 0.014$ & $-1.515 \pm 0.031$ & $-3.611 \pm 0.029$ \\
\hline & 0.0008 & $4.275 \pm 0.001$ & $1.924 \pm 0.002$ & $0.830 \pm 0.008$ & $0.186 \pm 0.014$ & $-1.270 \pm 0.025$ & $-3.405 \pm 0.036$ \\
\hline & 0.0018 & $4.746 \pm 0.001$ & $2.372 \pm 0.004$ & $1.200 \pm 0.011$ & $0.556 \pm 0.017$ & $-0.901 \pm 0.032$ & $-3.060 \pm 0.045$ \\
\hline & 0.0037 & $5.278 \pm 0.000$ & $2.878 \pm 0.001$ & \pm 0.005 & $1.008 \pm 0.005$ & $-0.468 \pm 0.010$ & $-2.696 \pm 0.020$ \\
\hline & 0.0075 & $5.978 \pm 0.000$ & $3.400 \pm 0.001$ & $2.142 \pm 0.006$ & $1.505 \pm 0.008$ & $0.009 \pm 0.010$ & $-2.347 \pm 0.012$ \\
\hline & 0.0150 & $7.146 \pm 0.001$ & $3.960 \pm 0.001$ & $2.655 \pm 0.001$ & $2.030 \pm 0.002$ & $0.555 \pm 0.005$ & $-1.756 \pm 0.019$ \\
\hline & 0.0200 & $7.677 \pm 0.001$ & $4.183 \pm 0.001$ & $2.847 \pm 0.002$ & $2.228 \pm 0.002$ & $0.755 \pm 0.003$ & $-1.562 \pm 0.010$ \\
\hline \multirow[t]{7}{*}{3} & 0.0004 & $2.741 \pm 0.001$ & $1.386 \pm 0.004$ & $0.447 \pm 0.008$ & $-0.250 \pm 0.010$ & $-1.880 \pm 0.019$ & $-4.200 \pm 0.020$ \\
\hline & 0.0008 & $2.998 \pm 0.001$ & $1.729 \pm 0.003$ & $0.745 \pm 0.009$ & $0.052 \pm 0.008$ & $-1.565 \pm 0.008$ & $-3.900 \pm 0.016$ \\
\hline & 0.0018 & $3.389 \pm 0.004$ & $2.159 \pm 0.004$ & $1.115 \pm 0.007$ & $0.415 \pm 0.009$ & $-1.221 \pm 0.009$ & $-3.623 \pm 0.069$ \\
\hline & 0.0037 & $3.897 \pm 0.001$ & $2.567 \pm 0.002$ & $4 \pm 0.001$ & $0.904 \pm 0.002$ & $-0.703 \pm 0.008$ & $-3.176 \pm 0.014$ \\
\hline & 0.0075 & $4.534 \pm 0.000$ & $2.912 \pm 0.001$ & $1.980 \pm 0.006$ & $1.353 \pm 0.006$ & $-0.214 \pm 0.012$ & $-2.716 \pm 0.028$ \\
\hline & 0.0150 & $5.540 \pm 0.000$ & $3.291 \pm 0.001$ & $2.439 \pm 0.002$ & $1.848 \pm 0.003$ & $0.346 \pm 0.006$ & $-2.071 \pm 0.010$ \\
\hline & 0.0200 & $6.037 \pm 0.001$ & $3.455 \pm 0.001$ & $2.619 \pm 0.002$ & $2.044 \pm 0.002$ & $0.566 \pm 0.007$ & $-1.827 \pm 0.020$ \\
\hline
\end{tabular}


A. Marín-Franch and A. Aparicio: Surface-brightness fluctuations in stellar populations, Online Material p 17

Table 13. Observational data: galaxies.

\begin{tabular}{|c|c|c|c|c|c|c|c|c|}
\hline Galaxy & $\bar{m}_{V}$ & $\overline{\bar{m}_{I}}$ & $\bar{m}_{K}$ & $\overline{\bar{m}_{F 814 W}}$ & $\overline{(V-I)_{0}}$ & $\overline{D D M}$ & {$[\mathrm{~F} / \mathrm{Fe} / \mathrm{H}]$} & Age (Gyr) \\
\hline NGC 0221 & $25.14 \pm 0.10$ & $22.73 \pm 0.05$ & $18.56 \pm 0.09$ & $22.84 \pm 0.03$ & $1.133 \pm 0.007$ & $24.56 \pm 0.17$ & -0.04 & 3.8 \\
\hline NGC 0224 & $25.29 \pm 0.08$ & $23.03 \pm 0.05$ & $18.80 \pm 0.21$ & $23.54 \pm 0.04$ & $1.231 \pm 0.007$ & $24.44 \pm 0.10$ & 0.42 & 5.1 \\
\hline NGC 1316 & $32.25 \pm 0.16$ & $29.83 \pm 0.15$ & & & $1.132 \pm 0.016$ & $31.28 \pm 0.09$ & 0.25 & 3.4 \\
\hline NGC 1399 & $32.47 \pm 0.12$ & $30.11 \pm 0.13$ & $25.98 \pm 0.16$ & & $1.227 \pm 0.016$ & $31.31 \pm 0.09$ & 0.50 & \\
\hline NGC 1404 & $32.48 \pm 0.12$ & $30.20 \pm 0.16$ & $25.82 \pm 0.14$ & & $1.224 \pm 0.016$ & $31.33 \pm 0.10$ & 0.47 & 5.9 \\
\hline NGC 3377 & $30.57 \pm 0.11$ & $28.35 \pm 0.06$ & & $28.59 \pm 0.05$ & $1.114 \pm 0.009$ & $30.19 \pm 0.08$ & 0.20 & 4.1 \\
\hline NGC 3379 & $31.21 \pm 0.06$ & $28.57 \pm 0.07$ & & $28.81 \pm 0.11$ & $1.193 \pm 0.015$ & $30.23 \pm 0.06$ & 0.16 & 9.3 \\
\hline NGC 4374 & $32.02 \pm 0.09$ & $29.77 \pm 0.09$ & & $30.02 \pm 0.01$ & $1.191 \pm 0.008$ & $31.20 \pm 0.10$ & 0.12 & 11.8 \\
\hline NGC 4472 & $32.26 \pm 0.06$ & $29.62 \pm 0.07$ & $25.30 \pm 0.11$ & $25.25 \pm 0.13$ & $1.218 \pm 0.011$ & $31.07 \pm 0.08$ & 0.24 & 8.5 \\
\hline NGC 4649 & & $29.76 \pm 0.09$ & & $30.11 \pm 0.09$ & $1.232 \pm 0.023$ & $31.09 \pm 0.08$ & 0.30 & \\
\hline
\end{tabular}


A. Marín-Franch and A. Aparicio: Surface-brightness fluctuations in stellar populations, Online Material p 18

Table 14. Observational data: globular clusters.

\begin{tabular}{cccccc}
\hline \hline Globular Cluster & $\bar{m}_{V}$ & $\bar{m}_{I}$ & $(V-I)_{0}$ & $D M$ & {$[\mathrm{Fe} / \mathrm{H}]$} \\
\hline NGC 104 & $13.15 \pm 0.08$ & $11.10 \pm 0.16$ & 1.06 & 13.37 & -0.76 \\
NGC 288 & $14.53 \pm 0.34$ & $12.57 \pm 0.45$ & 0.92 & 14.69 & -1.24 \\
NGC 2419 & $19.29 \pm 0.09$ & $17.72 \pm 0.12$ & 0.85 & 19.97 & -2.34 \\
NGC 5139 & $13.67 \pm 0.10$ & $12.01 \pm 0.12$ & 0.95 & 13.97 & -1.62 \\
NGC 5272 & $14.37 \pm 0.14$ & $12.59 \pm 0.17$ & 0.88 & 15.12 & -1.57 \\
NGC 5904 & $14.04 \pm 0.16$ & $12.30 \pm 0.19$ & 0.89 & 14.46 & -1.29 \\
NGC 6093 & $14.84 \pm 0.18$ & $12.90 \pm 0.21$ & 0.99 & 15.56 & -1.75 \\
NGC 6205 & $13.68 \pm 0.16$ & $12.05 \pm 0.20$ & 0.88 & 14.48 & -1.54 \\
NGC 6341 & $13.85 \pm 0.17$ & $12.23 \pm 0.20$ & 0.86 & 14.64 & -2.29 \\
NGC 6624 & $15.45 \pm 0.20$ & $12.40 \pm 0.27$ & 1.20 & 15.37 & -0.42 \\
NGC 6626 & $14.41 \pm 0.18$ & $11.86 \pm 0.22$ & 1.01 & 15.12 & -1.45 \\
NGC 6637 & $15.30 \pm 0.11$ & $12.91 \pm 0.19$ & 1.07 & 15.16 & -0.71 \\
NGC 6652 & $15.43 \pm 0.13$ & $13.48 \pm 0.22$ & 1.03 & 15.19 & -0.96 \\
NGC 6656 & $12.96 \pm 0.21$ & $10.83 \pm 0.26$ & 1.00 & 13.60 & -1.64 \\
NGC 6723 & $14.88 \pm 0.13$ & $13.23 \pm 0.22$ & 1.01 & 14.87 & -1.12 \\
NGC 6765 & $16.93 \pm 0.12$ & $15.16 \pm 0.13$ & 0.90 & 17.61 & -1.59 \\
NGC 7006 & $17.53 \pm 0.18$ & $16.03 \pm 0.21$ & 0.92 & 18.24 & -1.63 \\
NGC 7078 & $14.65 \pm 0.11$ & $13.06 \pm 0.13$ & 0.83 & 15.37 & -2.25 \\
NGC 7089 & $14.89 \pm 0.09$ & $13.29 \pm 0.11$ & 0.91 & 15.49 & -1.62 \\
\hline
\end{tabular}


A. Marín-Franch and A. Aparicio: Surface-brightness fluctuations in stellar populations, Online Material p 19

Table 15. Observational data: superclusters.

\begin{tabular}{ccccc}
\hline \hline Supercluster & $\bar{M}_{K_{\mathrm{S}}}$ & $(V-I)$ & $Z$ & Age $(\mathrm{Gyr})$ \\
\hline SWB V & $-6.69 \pm 0.20$ & $0.78 \pm 0.11$ & $0.008 \pm 0.004$ & 3 \\
SWB VI & $-6.21 \pm 0.24$ & $1.02 \pm 0.07$ & $0.004 \pm 0.002$ & 6 \\
SWB VII & $-4.92 \pm 0.38$ & $1.06 \pm 0.13$ & $0.0006 \pm 0.0003$ & 12 \\
\hline
\end{tabular}


A. Marín-Franch and A. Aparicio: Surface-brightness fluctuations in stellar populations, Online Material p 20

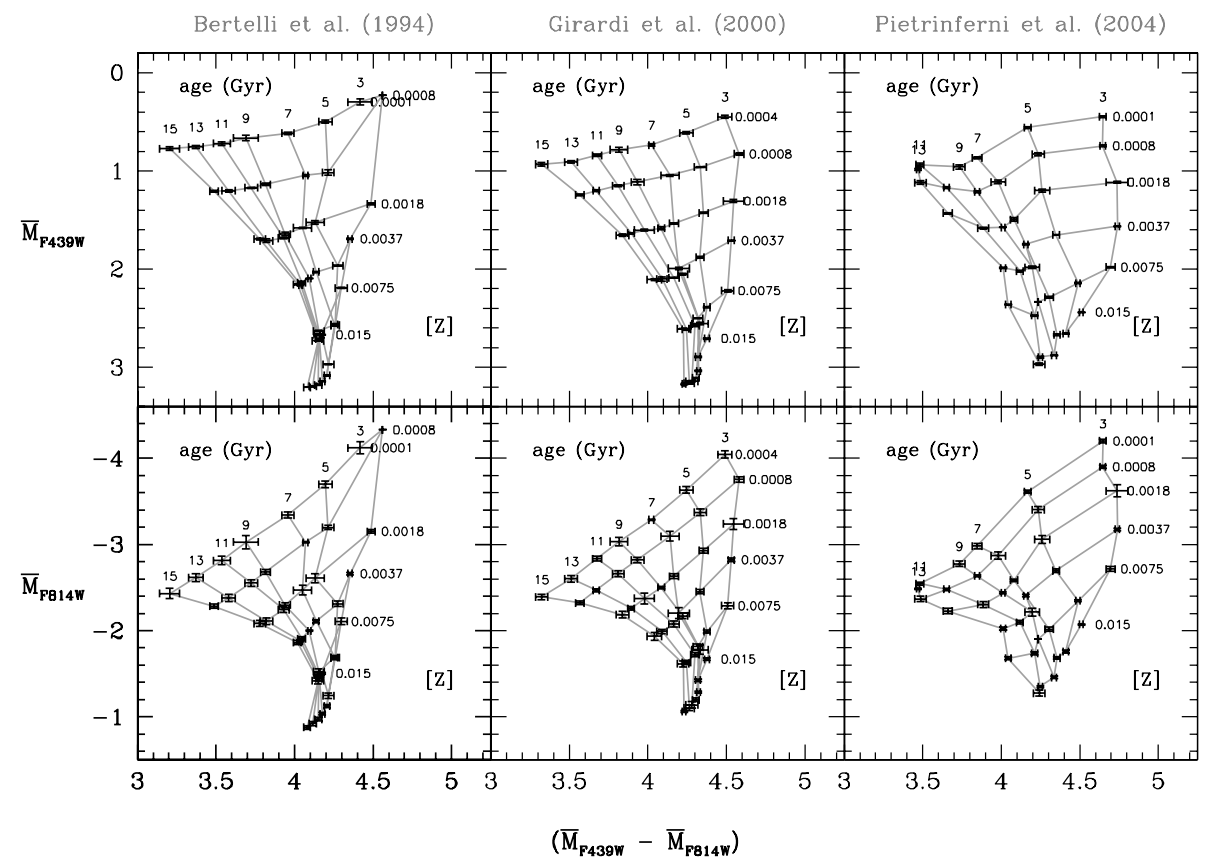

Fig. 5. Same as Fig. 3, but here $\bar{M}_{F 450 W}$ and $\bar{M}_{F 814 W}$ have been chosen, as an example of HST WFPC2 and ACS filters. As in Figs. 3 and 4 , the three stellar evolution libraries provide comparable results, being the G00 and P04 based model results are slightly redder than those based on B94. The age-metallicity degeneracy is broken in all cases for low metallicity $(Z \leq 0.0037)$ stellar populations. 
A. Marín-Franch and A. Aparicio: Surface-brightness fluctuations in stellar populations, Online Material p 21

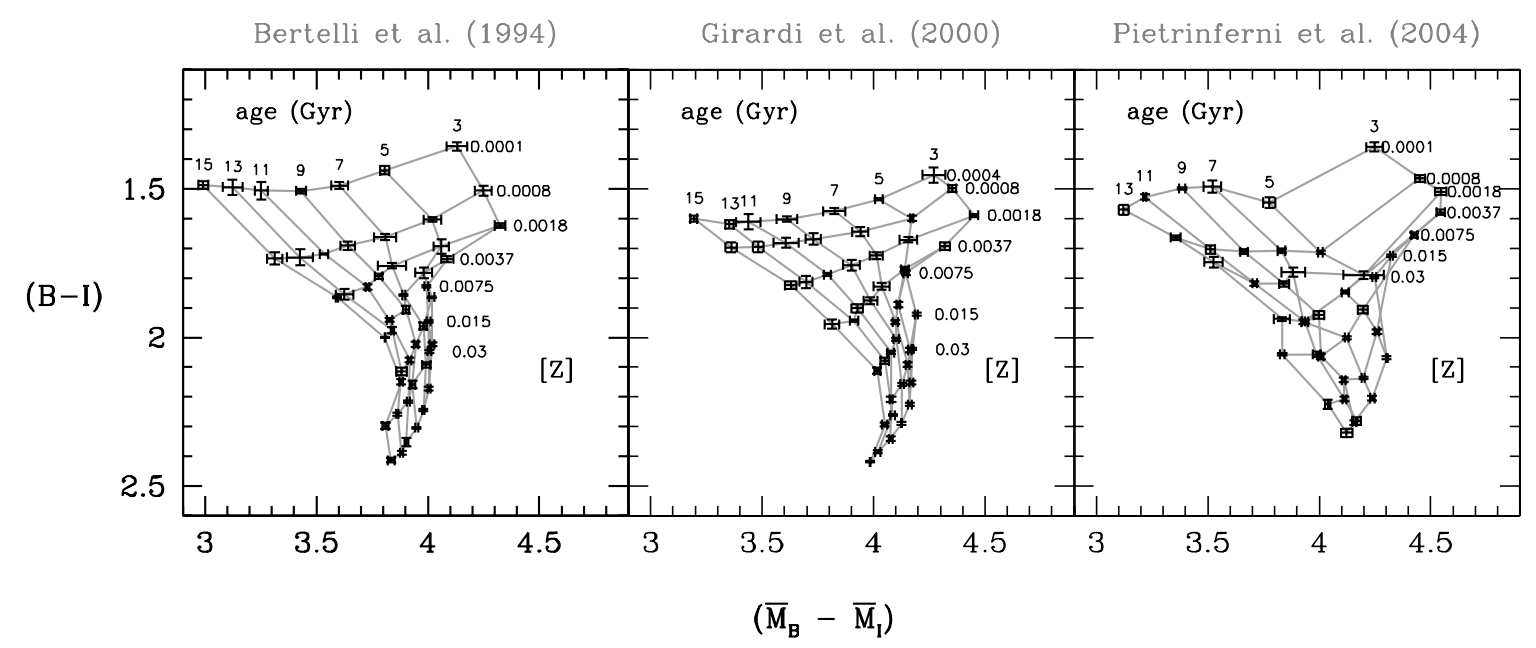

Fig. 6. Example of color \& SBF-color model grids obtained using respectively the stellar evolution libraries by B94, G00 and P04. Lines represent SSP with same age or metallicity. This plot provides less resolution that the one shown in Figs. 3 to 5, but having the advantage of being distance independent. So it can be very useful in those cases where distance is unknown or not well determined.

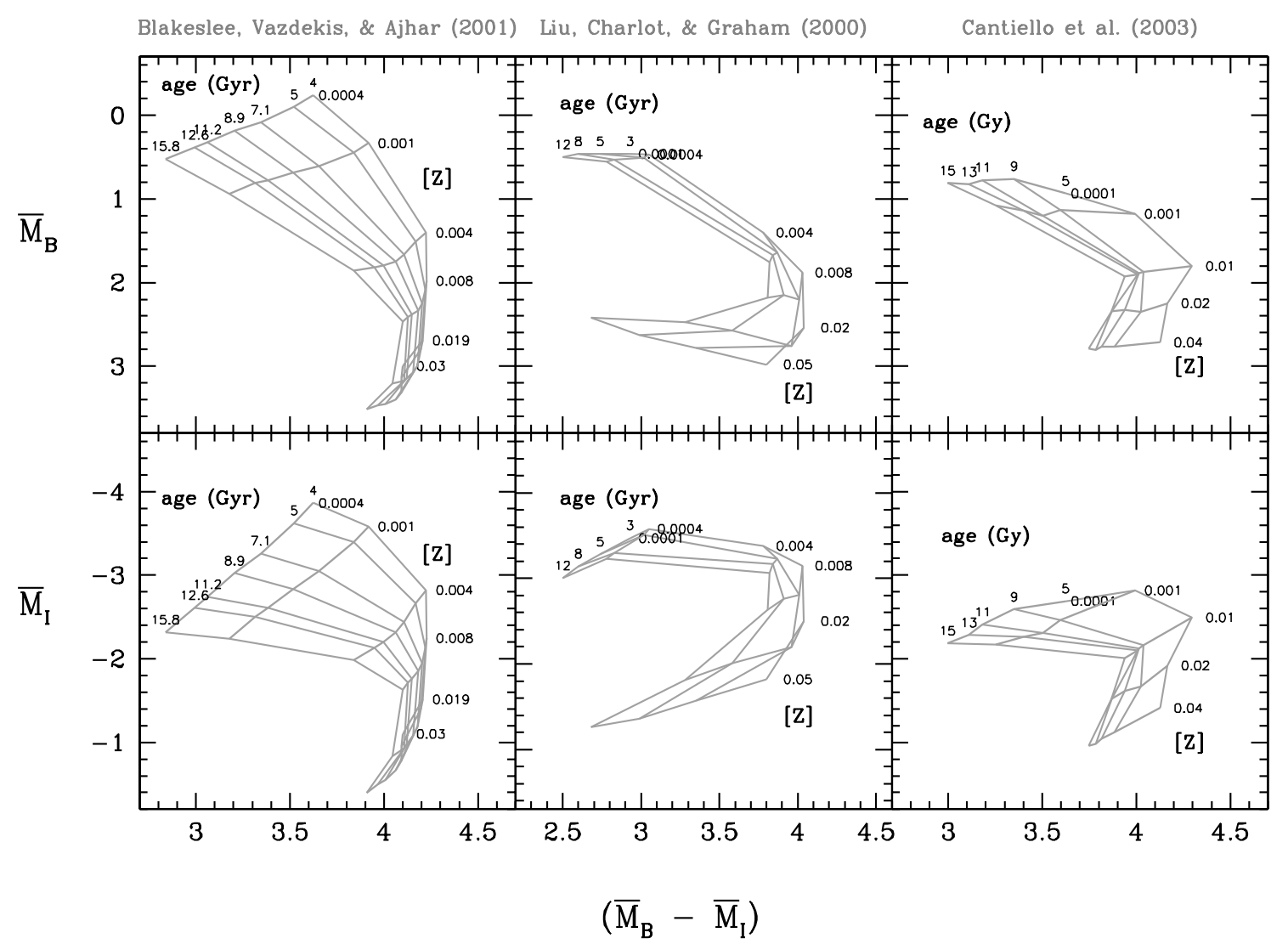

Fig. 7. Same as Fig. 3, but obtained from BVA01, LCG00 and C03. $\bar{M}_{B}$ and $\bar{M}_{I}$ SBF magnitudes have been chosen for comparison with our FCMDs. Note that, in LCG00 diagrams, the $X$-axis scale is different. It can be seen that the three sets of models show very different FCMDs. BVA01 SBF results show similar age and metallicity trends as ours, while LCG00 and C03 SBF results show a completely different behavior. 
A. Marín-Franch and A. Aparicio: Surface-brightness fluctuations in stellar populations, Online Material p 22

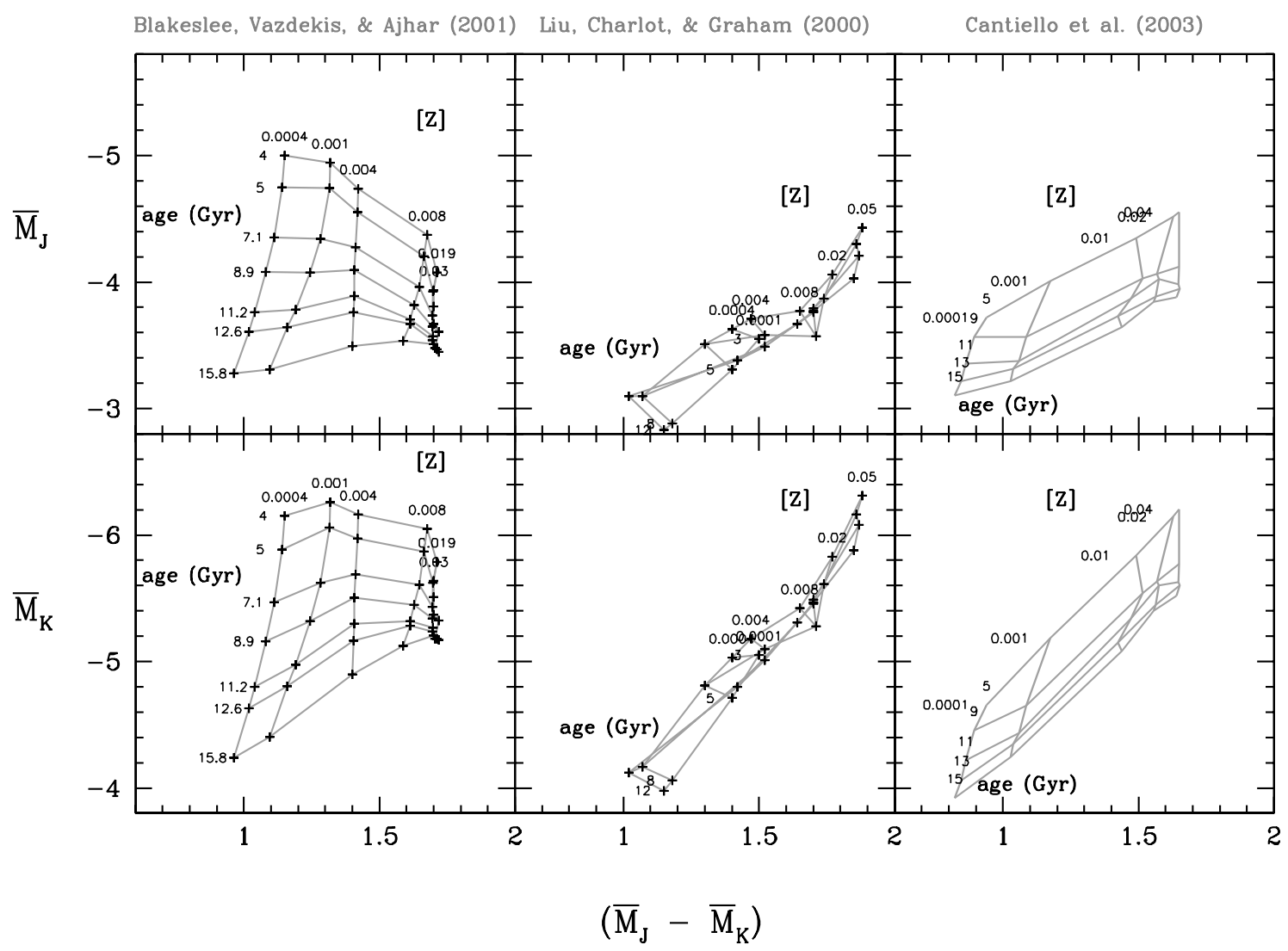

Fig. 8. Same as Fig. 3, but obtained from BVA01, LCG00 and C03. $\bar{M}_{J}$ and $\bar{M}_{K}$ SBF magnitudes have been chosen for comparison with our FCMDs. It can be seen that the three sets of models show very different calibration grids. As in the optical wavelengths, BVA01 SBF results show similar age and metallicity trends as ours, while LCG00 and C03 SBF results show a completely different behavior. 
A. Marín-Franch and A. Aparicio: Surface-brightness fluctuations in stellar populations, Online Material p 23

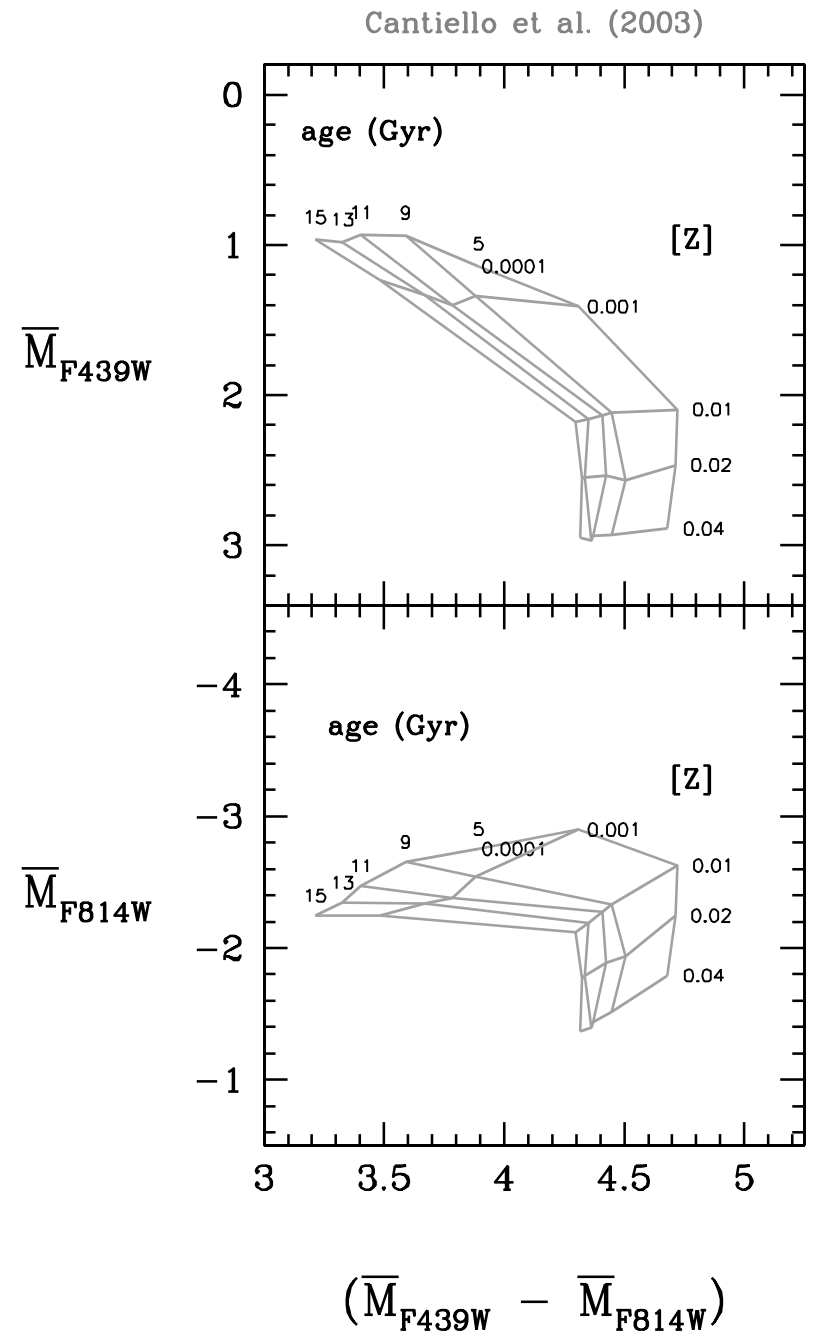

Fig. 9. HST WFPC2 and ACS filters: FCMDs obtained from $\mathrm{C} 03$. BVA01 and LCG00 results are not shown here because $F 439 W$ and F814W filters were not computed in their SBF calibrations. $\bar{M}_{F 429 W}$ and $\bar{M}_{F 814 W}$ SBF magnitudes have been chosen for comparison with our FCMDs. The trend here is the same found in Fig. 7. 
A. Marín-Franch and A. Aparicio: Surface-brightness fluctuations in stellar populations, Online Material p 24

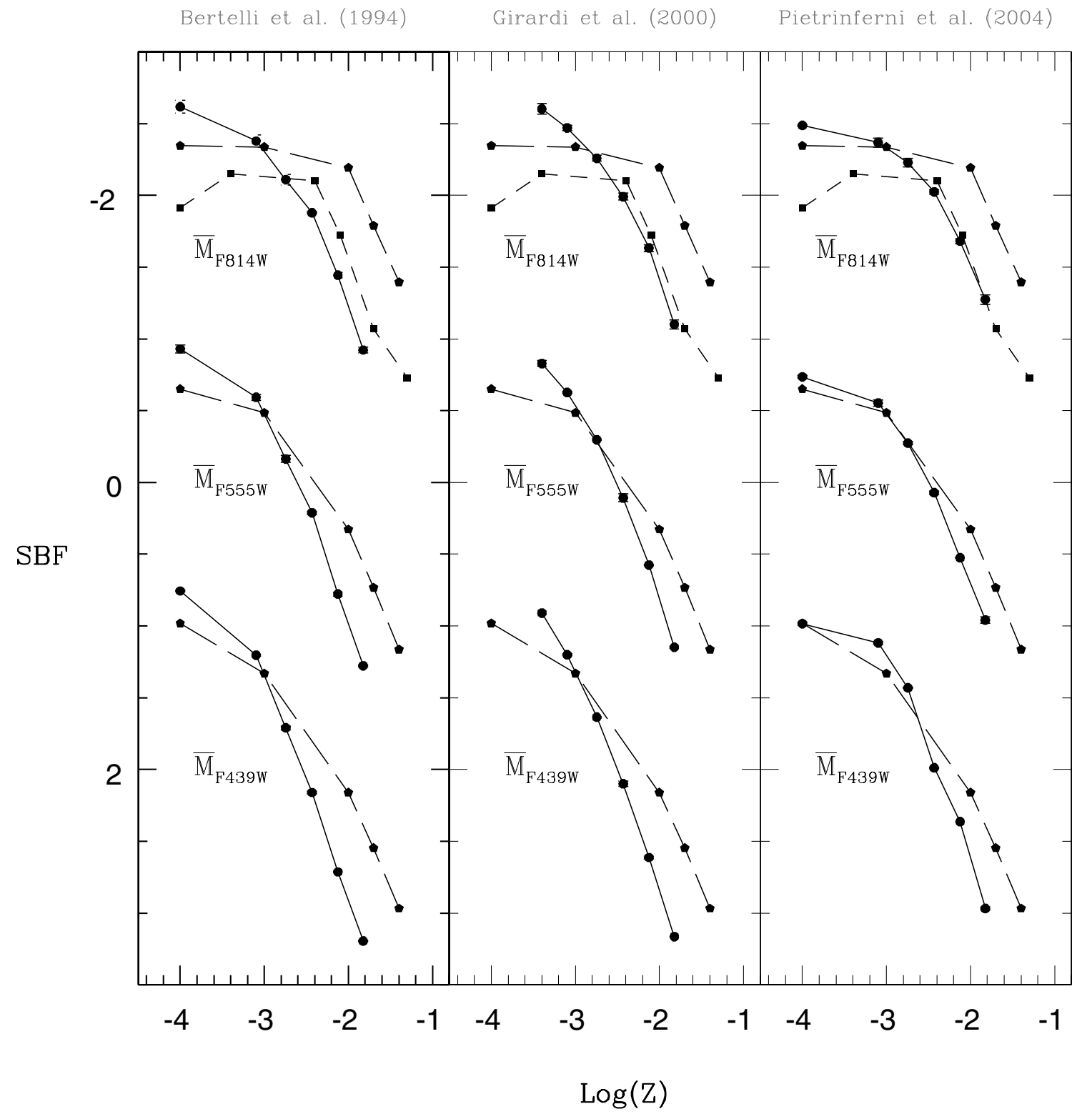

Fig. 11. Same as Fig. 10 but for the HST WFPC and ACS filters. Our 13 Gyr SBF results, based on B94, G00 and P04, respectively, are plotted as circles with solid line, LCG00 $12 \mathrm{Gyr}$ as squares with short dash, and C03 $13 \mathrm{Gyr}$ as pentagons with long dash. $\bar{M}_{F 439 W}, \bar{M}_{F 555 W}$ and $\bar{M}_{F 814 W}$ have been considered in this plot. Here the trends are equivalent as those shown in Fig. 10. 
A. Marín-Franch and A. Aparicio: Surface-brightness fluctuations in stellar populations, Online Material p 25

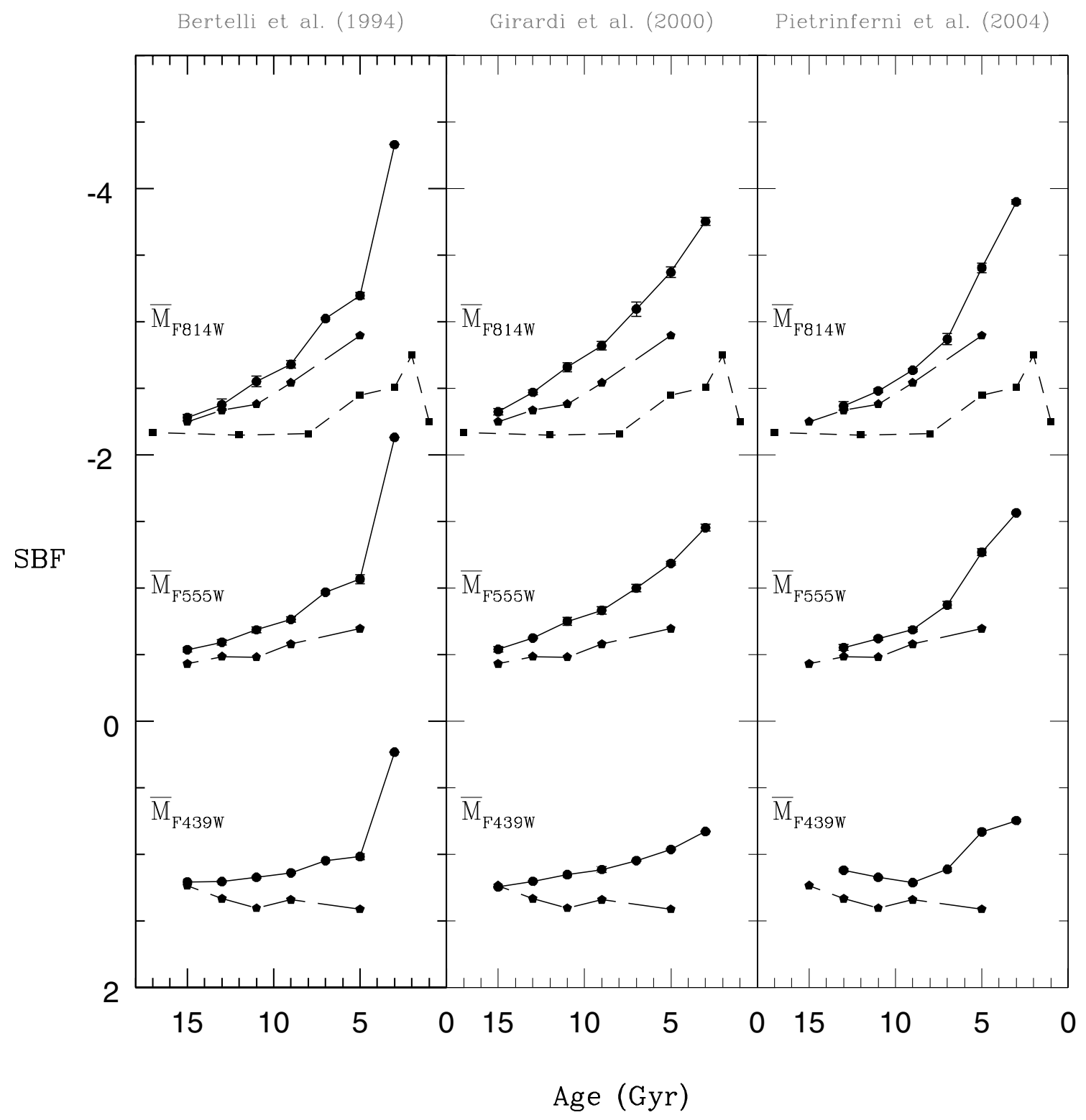

Fig. 13. Same as Fig. 12 but for the HST WFPC and ACS filters. Our SBF results, based on B94, G00 and P04, respectively, are plotted as circles with solid line, LCG00 as squares with short dash, and C03 as pentagons with long dash. $\bar{M}_{F 439 W}, \bar{M}_{F 555 W}$ and $\bar{M}_{F 814 W}$ have been considered in this plot. Here the trends are equivalent as those shown in Fig. 10. 
A. Marín-Franch and A. Aparicio: Surface-brightness fluctuations in stellar populations, Online Material p 26

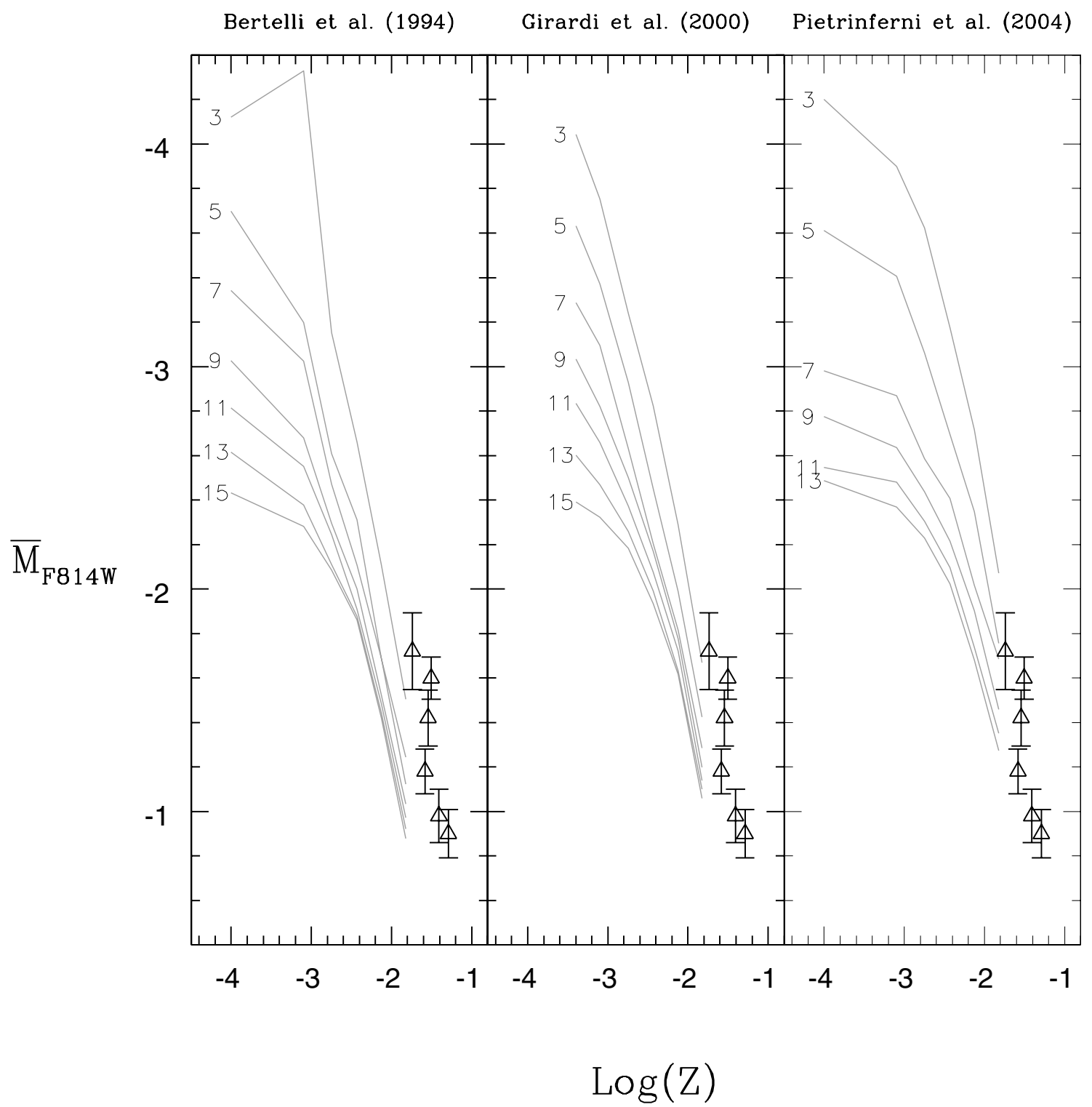

Fig. 19. Same as Fig. 21, but for the $F 814 W$ filter. Open triangles the galaxies listed in Table 13. Again, P04 based models are the only able to reproduce galaxy data. 